Florida International University FIU Digital Commons

$3-27-2008$

\title{
Synthesis of copper carbon nanotube composite and its electrical conductivity measurement
}

Sushma Amruthaluri

Florida International University

DOI: $10.25148 /$ etd.FI14032310

Follow this and additional works at: https://digitalcommons.fiu.edu/etd

Part of the Materials Science and Engineering Commons

\section{Recommended Citation}

Amruthaluri, Sushma, "Synthesis of copper carbon nanotube composite and its electrical conductivity measurement" (2008). FIU Electronic Theses and Dissertations. 1283.

https://digitalcommons.fiu.edu/etd/1283

This work is brought to you for free and open access by the University Graduate School at FIU Digital Commons. It has been accepted for inclusion in FIU Electronic Theses and Dissertations by an authorized administrator of FIU Digital Commons. For more information, please contact dcc@fiu.edu. 
FLORIDA INTERNATIONAL UNIVERSITY

Miami, Florida

SYNTHESIS OF COPPER CARBON NANOTUBE COMPOSITE AND ITS

ELECTRICAL CONDUCTIVITY MEASUREMENT

A thesis submitted in partial fulfillment of the

requirements for the degree of

MASTER OF SCIENCE

in

MATERIALS SCIENCE AND ENGINEERING

by

Sushma Amruthaluri

2008 
To: Interim Dean Amir Mirmiran

College of Engineering and Computing

This thesis, written by Sushma Amruthaluri, and entitled Synthesis of Copper Carbon Nanotube Composite and its Electrical Conductivity Measurement, having been approved in respect to style and intellectual content, is referred to you for judgment.

We have read this thesis and recommend that it be approved.

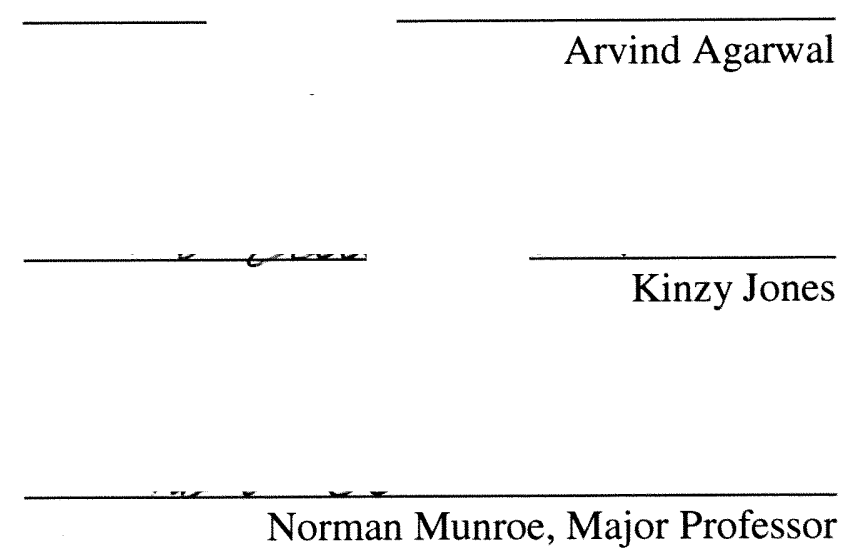

Date of Defense: March 27, 2008

The thesis of Sushma Amruthaluri is approved.

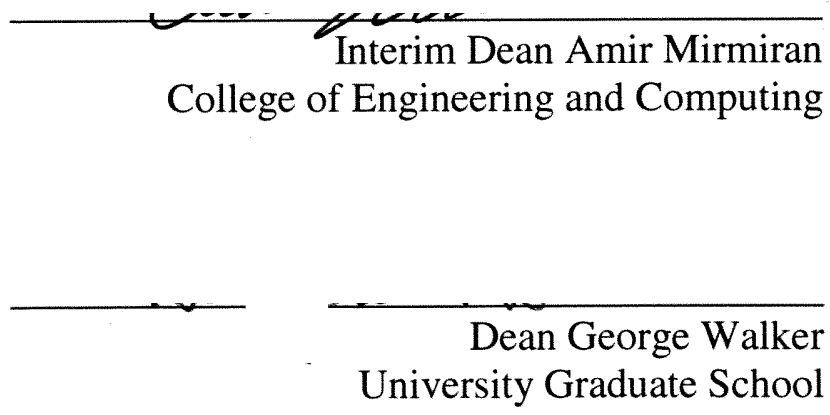

Florida International University, 2008 


\section{DEDICATION}

I dedicate this thesis to my mother Shakira Begum, my sister Reshma Thummadi and my brother-in-law Avinash Thummadi. Without their patience, understanding, support and most of all love, the completion of this work would not have been possible. 


\section{ACKNOWLEDGMENTS}

I wish to thank all the members of the committee for their invaluable support and encouragement throughout my sojourn. Their gentle but firm direction has been most appreciated. First of all, I wish to acknowledge my Major Professor, Dr. Norman Munroe for the guidance and the mentoring he provided. I extend my gratitude to Dr. Arvind Agarwal and Dr. Kinzy Jones for their invaluable support in guiding me towards a qualitative methodology. Last but not least, I extend my profound gratitude to my friends Puneet Kamal Singh Gill, Kantesh Balani and Waseem Haider for assisting and advising me all the way long. 


\section{ABSTRACT OF THE THESIS \\ SYNTHESIS OF COPPER CARBON NANOTUBE COMPOSITE AND ITS \\ ELECTRICAL CONDUCTIVITY MEASUREMENT}

by

Sushma Amruthaluri

Florida International University, 2008

Miami, Florida

Professor Norman Munroe, Major Professor

The matrices in which Multi Walled Carbon Nanotubes (MWCNTs) are incorporated to produce composites with improved electrical properties can be polymer, metal or metal oxide. Most composites containing CNTs are polymer based because of its flexibility in fabrication. Very few investigations have been focused on CNT-metal composites due to fabrication difficulties, such as achievement of homogeneous distribution of MWCNTs and poor interfacial bonding between MWCNTs and the metal matrix. In an effort to overcome poor interfacial bonding for the $\mathrm{Cu}-\mathrm{MWCNT}$ composite, silver $(\mathrm{Ag})$ and nickel $(\mathrm{Ni})$ resinates have been incorporated in the ball milling stage. Composites of MWCNT $(16,12$, and $8 \mathrm{Vol} \%)-\mathrm{Cu}+\mathrm{Ag}+\mathrm{Ni}$ were pelleted at 20,000 psi (669.4 Mpa) and sintered at $950{ }^{\circ} \mathrm{C}$. The electrical conductivity results measured by four probe meter showed that the conductivity decreases with increase in the porosity. Moreover from these results it can also be stated that an addition of optimum value of (12 Vol \%) MWCNT leads to high electrical conductivity $\left(9.26 \mathrm{E}+07 \mathrm{~s}-\mathrm{m}^{-1}\right)$, which is $50 \%$ greater than the conductivity of $\mathrm{Cu}$. It is anticipated that the conductivity can be increased substantially with hot isostatic pressing of the pellet. 


\section{TABLE OF CONTENTS}

CHAPTER

PAGE

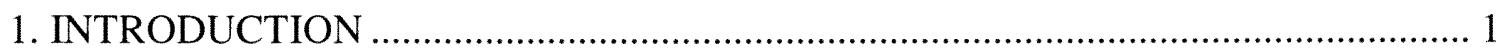

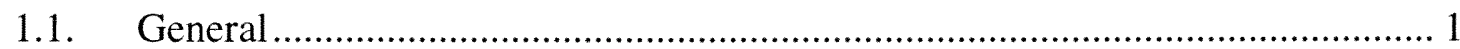

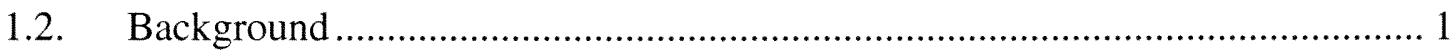

1.3. Drawbacks of $\mathrm{Cu}-\mathrm{MWCNT}$ Composites .......................................................... 4

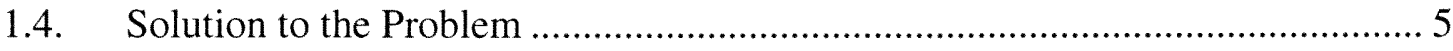

1.4.1. Homogenous Distribution of MWCNT ..................................................... 5

1.4.2. Interfacial Bonding …........................................................................ 5

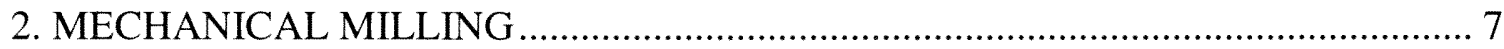

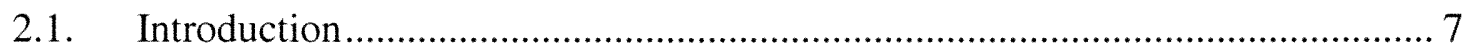

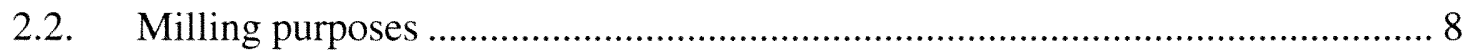

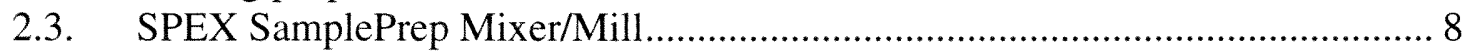

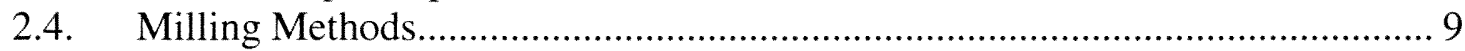

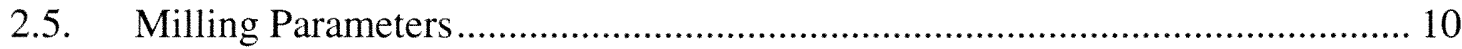

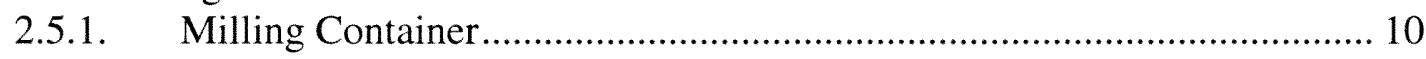

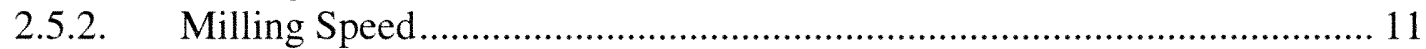

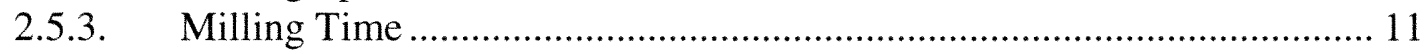

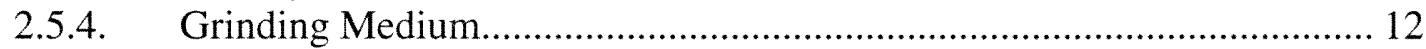

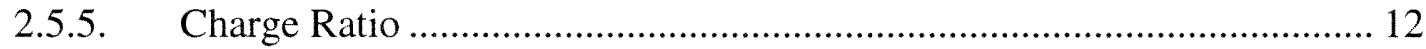

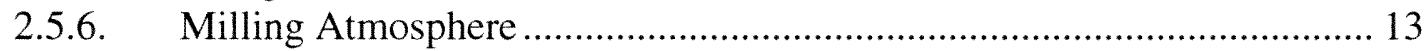

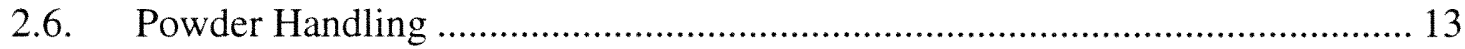

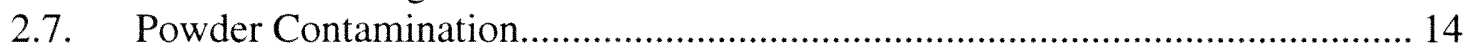

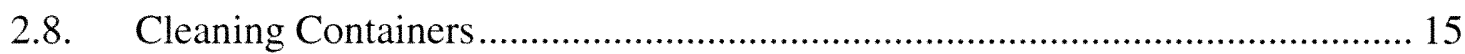

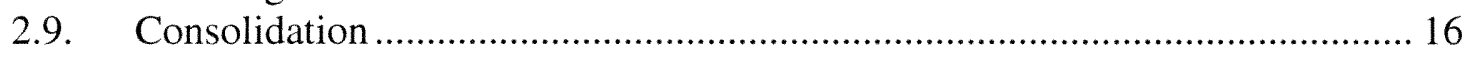

3. POROSITY …

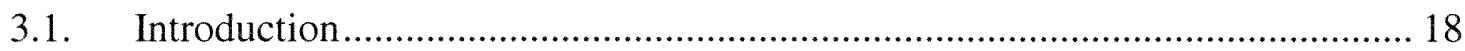

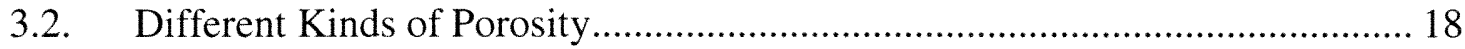

3.3. Parameters Influencing Porosity ………...................................................... 19

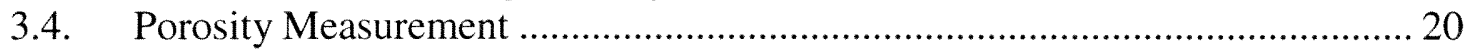

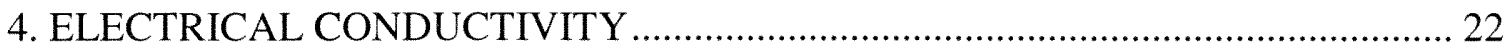

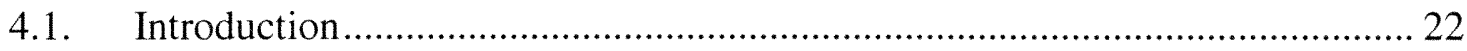

4.2. Effect of Temperature on Electrical Conductivity ......................................... 22

4.3. Effect of Porosity on Electrical Conductivity .................................................. 23

4.4. Electrical Resistivity Measurement .................................................................. 23

4.5. Limits of Measurement Capability ………................................................... 26

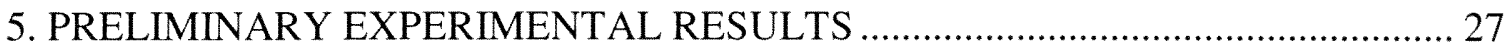

5.1. Selection of Milling Time of Composite Materials .......................................... 27

5.2. Verifying the Effect of Ball Milling on Electrical Conductivity ...................... 29 
6. PREPARATION OF COPPER-CARBON NANOTUBE COMPOSITE …............... 33

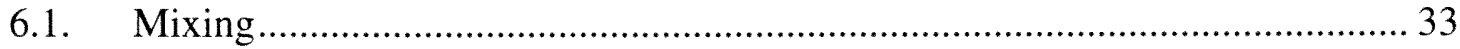

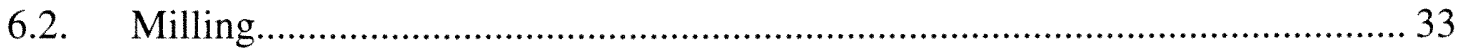

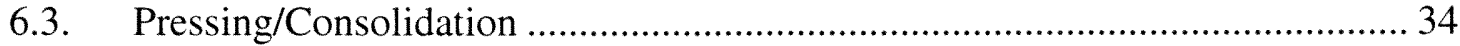

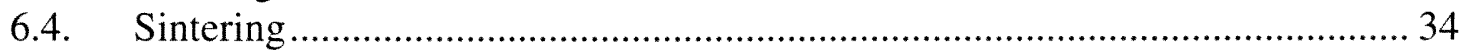

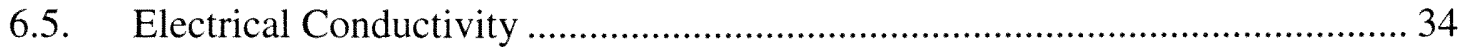

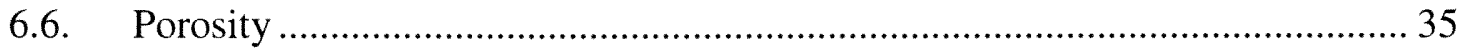

6.7. Morphology of Cu-MWCNT composites ..................................................... 36

6.8. Conclusions from preliminary experiments ...................................................... 39

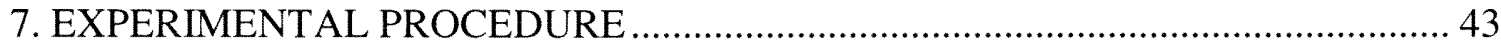

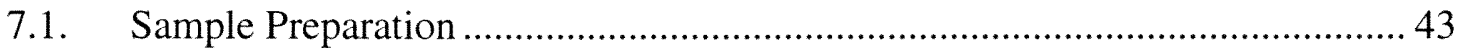

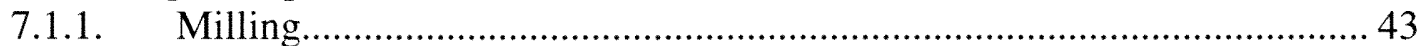

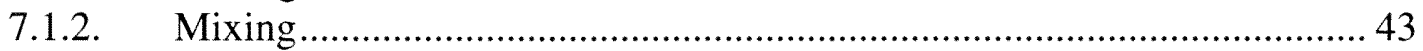

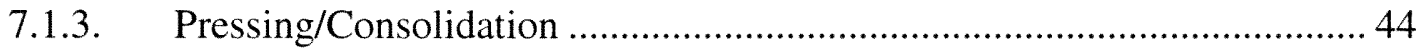

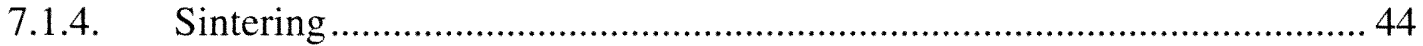

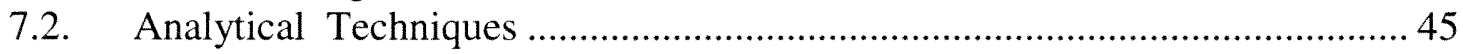

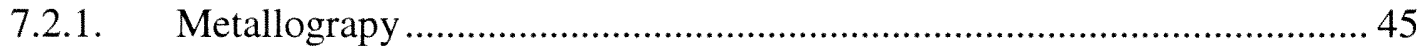

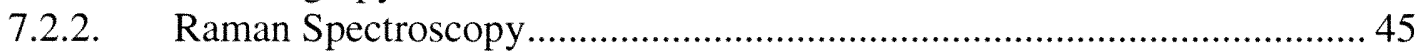

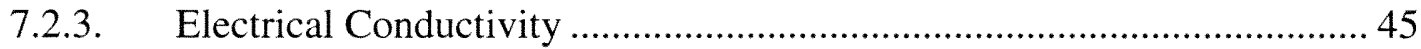

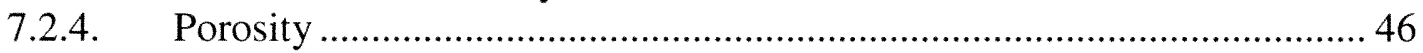

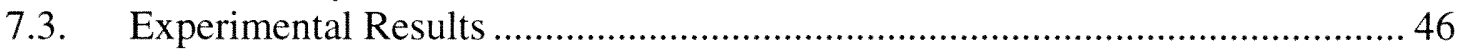

7.3.1. Electrical Resistance Graphs of Un-Sintered Pellets ................................46

7.3.2. Electrical Resistance Graphs of sintered Pellets ...................................... 47

7.3.3. Raman Spectroscopy Analyses .............................................................. 51

7.3.4. EDS Analysis and X-Ray mapping of Un-sintered Samples...................5 54

7.3.5. SEM and EDS Analysis of Sintered Composites .................................... 58

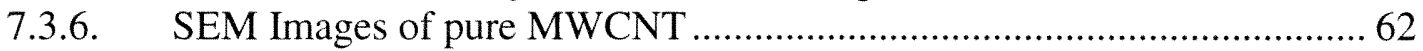

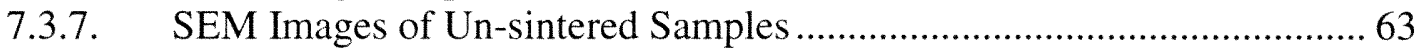

7.3.8. SEM Images of Sintered Samples.............................................................. 64

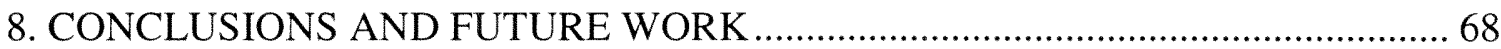

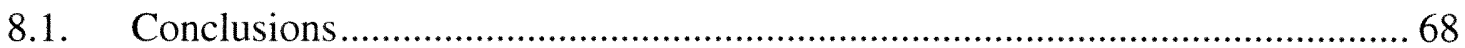

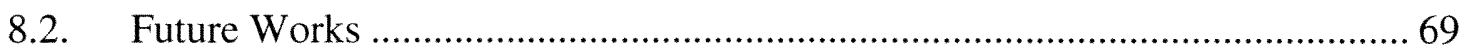

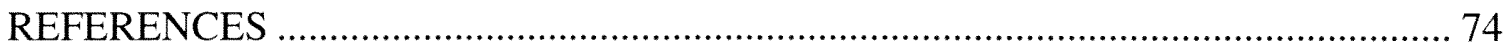

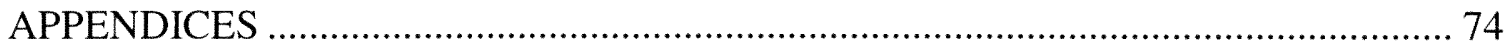




\section{LIST OF TABLES}

\section{TABLE}

PAGE

Table 5.1: Comparision of electrical resistivity and conductivity 30

Table 5.2: Porosity of various copper materials......................................................... 32

Table 6.1: Composition of Cu-MWCNT composites studied...................................... 33

Table 6.2: $\quad$ Process parameters and conductivity of re-sintered Cu-MWCNT composites

Table 6.3: $\quad$ Process parameters and conductivity of $\mathrm{Cu}-\mathrm{MWCNT}$ composites .......... 36

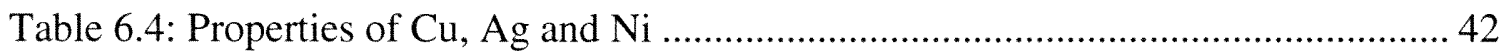

Table 7.1: Weight of individual elements in the composite pellet sample ................ 44

Table 7.2: Electrical resistivity and conductivity results of sintered and un-sintered CuAgNi-MWCNT composites

Table 7.3: Porosity results of sintered and un-sintered pellets..................................50

Table 7.4: Raman spectroscopy of $16 \mathrm{Vol} \%$ of MWCNT in the composite.............. 52

Table 7.5: $\quad \mathrm{D}$ and G peaks of MWCNT and $12 \mathrm{Vol} \%$ composites ........................... 53 


\section{LIST OF FIGURES}

FIGURE

PAGE

Figure 2.1: Hardened steel vial set with stainless steel ball set ............................... 10

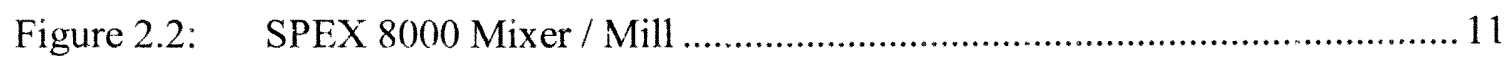

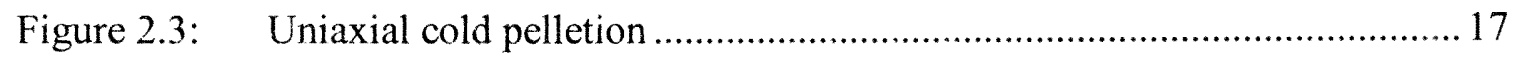

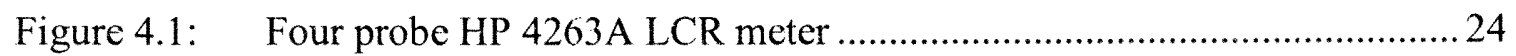

Figure 4.2: Schematic representation of the Four-point probe …...............................2.24

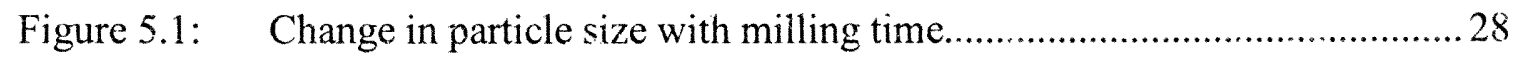

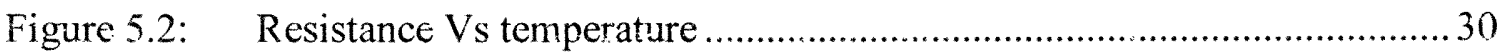

Figure 5.3: Variation of electrical resistivity ........................................................ 31

Figure 5.4: Variation of electrical conductivity........................................................ 31

Figure 6.1 (a): SEM images of Cu-MWCNT composites............................................ 37

Figure 6.1 (b): $\quad$ SEM images of Cu-MWCNT composites............................................. 38

Figure 6.1 (c): SEM images of Cu-MWCNT composites............................................ 38

Figure 6.2 (a): Low magnification SEM morphology images of Cu-MWCNT

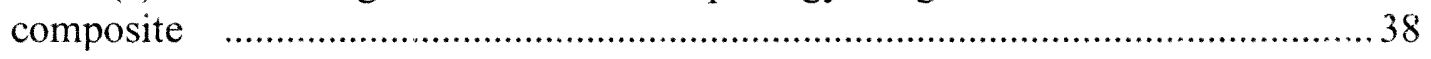

Figure 6.2 (b): Low magnification SEM morphology images of Cu-MWCNT composite

Figure 6.3: (a) $\mathrm{Cu}-\mathrm{C}$ phase diagram showing the solubility of carbon in copper ............ 40

Figure 6.3:(b) Cu-C phase diagram showing the solubility of carbon in copper.............41

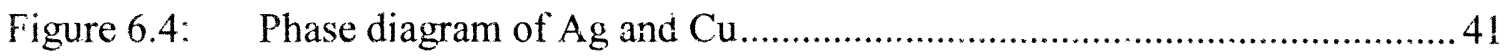

Figure 7.1: Electrical Resistance of un-sintered pellets......................................... 47

Figure 7.2: $\quad$ Electrical Resistance graphs of sintered pellets .....................................48

Figure 7.3: Electrical conductivity of sintered and Un-sintered composites...............50 
Figure 7.4: Electrical conductivity of sintered and $U$ n-sintered composites .............. 51

Figure 7.5: Raman spectroscopy of $16 \mathrm{Vol} \%$ of MWCNT in the composite..............52

Figure 7.6: Raman spectroscopy of $12 \mathrm{Vol} \%$ of MWCNT in the composite..............53

Fig 7.7: $\quad$ EDS analysis of un-sintered $16 \mathrm{Vol} \% \mathrm{MWCNT}$ composite......................... 54

Figure 7.8: X-Ray mapping of un-sintered 16 Vol \% MWCNT composite................. 55

Fig 7.9: EDS analysis of un-sintered $12 \mathrm{Vol} \% \mathrm{MWCN \Gamma}$ composite .......................56

Fig 7.10: EDS analysis of un-sintered $8 \mathrm{Vol} \%$ MWCNT composite ..........................57

Figure 7.11: X-Ray mapping of un-sintered 8 Vol \% MWCNT composite...................58

Fig 7.13: EDS analysis of sintered $16 \mathrm{Vol} \%$ MWCNT composite .............................58

Figure 7.14: X-Ray mapping of sintered 16 Vol\% MWCNT composite ......................59

Fig 7.15: EDS analysis of sintered $12 \mathrm{Vol} \%$ MWCNT composite .............................60

Figure 7.16: X-Ray mapping of sintered 12 Vol\% MWCNT composite ......................61

Figure 7.17: X-Ray mapping of sintered 8 Vol\% MWCNT composite …..................62 62

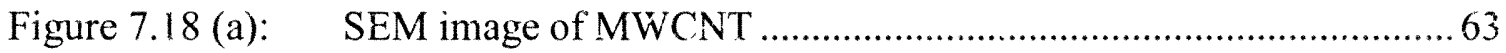

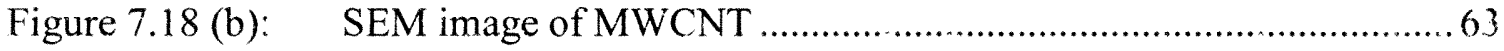

Figure 7.19 (a): $\quad$ SEM image of CuAgNi-MWCN'T composite.....................................6

Figure 7.19 (b): $\quad$ SEM image of CuAgNi-MWCNT composite..................................64

Figure 7.20 (a): $\quad$ SEM image of sintered CuAgNi-MWCNT composite ......................65

Figure 7.20 (b): SEM image of sintered CuAgNi-MWCNT composite ......................65

Figure 7.20 (c): $\quad$ SEM image of sintered CuAgNi-MWCNT composite ......................66

Figure $7.20(\mathrm{~d}): \quad$ SEM image of sintered CuAgNi-MWCNT composite ....................66

Figure 7.20 (e): SEM image of sintered CuAgNi-MWCNT composite .....................66 66

Figure 7.21: Schematic diagram of MWCNT' arrangement in the composite ............67 
Symbol DEFINITION

R Resistivity

P Porosity

T Temperature

W Weight

S

$\mathrm{t}$

$\mathrm{Al}$

Ar

$\mathrm{Cu}$

$\mathrm{Ag}$

$\mathrm{Ni}$

$\mathrm{O}$

O

$\rho$

$\sigma$ or $\kappa$ or $\gamma \quad$ Conductivity

$\alpha$

$\varphi$

Temperature Coefficient

Diameter

Aluminum

Argon

Copper

Silver

Nickel

Oxygen

Density
SI UNITS

ohm-m

${ }^{\circ} \mathrm{C}$

grams

$\mathrm{cm}$

$\mathrm{cm}$

\section{GREEK SYMBOLS}

$\mathrm{gms} / \mathrm{cm}^{3}$

$\mathrm{S} / \mathrm{m}$

inch 


\section{LIST OF ACRONYMS}

BPR

CNT

CR

CVD

INCS

MMC

PANI

IACS

PM

PPy

SEM

$\mathrm{XRF}$

rpm

MWCNT

HEBM

EDS

LCR meter
Balls-to-Powder Ratio

Carbon Nanotubes

Charge Ratio

Chemical Vapor Deposition

International Annealed Copper Standard

Metal Matrix Composite

Polyaniline

International Annealed Copper Standard

Powder Metallurgy

Polypyrrole

Scanning Electron Microscope

X-ray Fluorescence

rotations per minute

Multiwall Carbon Nanotubes

High Energy Ball Milling

Energy Dispersive Spectrometer

Inductance Capacitance Resistance meter 


\section{INTRODUCTION}

\subsection{General}

Copper $(\mathrm{Cu})$ and Aluminum (Al) have been the two dominating conductor materials during the past 100 years [1]. In the high technology applications, Silver $(\mathrm{Ag})$ is sometimes used because its conductivity is approximately 5 percent higher than that of $\mathrm{Cu}$. However, $\mathrm{Ag}$ is a much more expensive material [1]. A new conductor material with room temperature conductivity much higher than $\mathrm{Al}, \mathrm{Cu}$ and $\mathrm{Ag}$ would have technological and economical impact. Superconducting wires carry up to five times the current carried by conventional copper wires that have the same cross section [2]. Future demands for electricity will require the development not only of high-temperature superconducting wires but also, superconducting motors, transformers and generators with the advantages of reduction in weight, volume and losses as compared to their conventional counterparts. Incremental advances in existing grid technology are not capable of solving the urban power requirements envisaged in many urban settings. Therefore, revolutionary new materials will be required for high-temperature superconductivity. In this regard, an attempt is made to investigate the electrical

conductivity of Cu-MWCNT composites as a precursor to developing a highly conductive material.

\subsection{Background}

CNTs are a novel form of carbon that have the potential for spearheading exciting applications in the development of ultra strong wires, nanoelectronic devices, field electron emitters, nanoprobes, nanocomposite materials, among others [2-4]. CNTs 
are recognized as the ultimate carbon fiber for high performance, multifunctional composites [5 and 6]. Additionally, they can be used as potential reinforcements for composite materials. Since their discovery by Iijima in 1991 [7], CNTs have been the focus of considerable research due to their air stability, high aspect ratio, small diameter, lightweight, excellent mechanical, electrical, thermal and magnetic properties [8 and 9].

With the development of many low-cost synthetic routes for CNTs, such as chemical vapor deposition (CVD), arc discharge, laser ablation, and solvothermal processing, etc., mass production of CNTs has proven to be less cost-prohibitive [10 and 11]. Theoretical and experimental results have shown CNTs to have superior electriccurrent-carrying capacity 1000 times higher than that of copper wires [12]. High electrical conductivity is ultimately a nanoscale phenomenon comprised of two components - copper pairs which influence the superconducting state and Abrikosov vortices [2, other ref.], which mediate its current carrying ability. Nevertheless, CNTs are utilized here as an additive to improve the electrical properties of a composite.

The matrices used in CNTs-incorporated composites for improved electrical properties can be polymer, metal or metal oxide [13-16]. Most CNT composites are based on the usage of polymer matrices because of their flexibility and ease of fabrication. Chen et al. [13 ] utilized an electrochemical route for the formation of CNT-polypyrrole (PPy), which may have potential applications in CNT-based nanoelectronic structures. Zengin et al. [14] reported the preparation of CNT-polyaniline (PANI), which increased the electrical conductivity an order of magnitude over that of neat PANI. 
CNT-metal or CNT-metal oxide composites have been synthesized for improved electrical conductivity [14]. Peigney et al. synthesized CNT-metal oxide composites by hot-pressing the corresponding composite powders. The composites were found to be electrical conductors owing to the percolation of the nanotubes [15]. In general, only a few limited studies have been reported on metal matrix composites (MMCs), largely because of harsh fabrication conditions that compromise the integrity of the CNTs. Some established techniques employed in the fabricating of CNT MMCs include powder metallurgy (PM) [17-21], casting [22], and plasma spraying [23], where CNTs are subjected to severe conditions, of high temperature and pressure, during the process. Thus, the chemical stability of CNTs in MMCs has become one of the most challenging problems encountered in its manufacture.

A tremendous amount of research have been conducted on $\mathrm{Cu}$ matrix composites with additions of CNTs or carbon to impart low thermal expansion, high electrical and thermal conductivity coefficients for usage in electrical and contact materials such as brushes in electric motors, generators and engines [17 and 18]. Cu-CNT composites combine the positive characteristics of both components, i.e. good thermal and electrical conductivities from $\mathrm{Cu}$ and low thermal expansion coefficient and lubricating properties of graphite [24]. In a recent study of CNT reinforced $\mathrm{Cu}$ matrix nanocomposites by Cha et al [16], physically long, thin, and electrically conducting MWCNTs were shown to create an interconnected percolating network of conducting paths through the $\mathrm{Cu}$ matrix to yield high conductivity at relatively low MWCNT volume fractions. 


\subsection{Drawbacks of Cu-MWCNT Composites}

The Cu-MWCNT composites prepared by the powder metallurgy technique [1518] offers the possibility of obtaining a uniform product with a reduction in tedious and costly machining. However, this technique has the limitations of poor affinity between copper and MWCNTs, which has a detrimental effect on the structural, mechanical and electrical properties of the material [15-19]. Additionally, there are two important processing issues encountered in the fabrication of MWCNT reinforced $\mathrm{Cu}$ matrix composite materials; a) acquiring a homogeneous distribution of MWCNTs and b) achieving high interfacial bonding between MWCNTs and the Cu matrix [25].

The difficulty of distributing CNTs homogeneously in a $\mathrm{Cu}$ matrix has been regarded as one of the impeding hurdles $[32,26-28]$ in the manufacture of this composite, and many researchers are focused on solving this problem [31]. The same can be said about interfacial bonding and its influence on mechanical properties [25 and 29]. In a study of carbon fiber reinforced copper composites by Sun and Zhang [29], weak mechanical bonding due to poor wettability [30] was observed at the interface between the carbon fiber and the copper matrix. Thus, improving the interfacial bonding between the CNTs and metal matrix has become an important feature in the development of advanced engineering composites with improved mechanical and electrical properties [31- 37].

Structural damage or destruction of the nanotubes is often a problem encountered in the manufacture of Cu-MWCNT composites as a result of high processing temperatures and high pressures associated with the methods of producing metal 
matrices. Also, limited availability of MWCNTs has also restricted research efforts, although this seems to have become less of a problem in recent years as more researchers produce their own nanotubes via catalyzed chemical vapor deposition (CVD) methods.

\subsection{Solution to the Problem}

\subsubsection{Homogenous Distribution of MWCNT}

Although powder processing methods have been used to fabricate metal/CNT composites [38-46], the small diameter and large aspect ratio of the nanotubes render it difficult to obtain a homogenous mixture of the two phases prior to sintering or hot pressing. Nevertheless some success has been achieved with conventional milling techniques, primarily with the use of low to moderate nanotube volume fractions $[39,43$ 46]. In the current research a high speed SPEX SamplePrep Mixer/Mill was used to fabricate the Cu-MWCNT composite.

\subsubsection{Interfacial Bonding}

Small amount of alloying elements that have the characteristic nature of wettability were added in order to increase the interfacial bonding between MWCNT and copper matrix in the current research. It should be noted that the addition of a substance to improve one property may have unintended effects on other properties. Thus, small amounts of alloying elements to metals like copper may increase or reduce the strength, hardness, electrical and thermal conductivity, corrosion resistance, or change the color of a metal.

Copper by itself is relatively soft compared with common structural metals. However, when alloyed with elements such as tin, zinc and some other transition 
elements the strength of the resulting alloy is increased. Tin is more effective in strengthening copper, but is also more expensive and has a greater detrimental effect on the electrical and thermal conductivities. Aluminum, manganese, nickel and silicon additions also strengthen copper.

Pure copper is a very good conductor of both electricity and heat. The International Annealed Copper Standard (IACS; a high purity copper with a resistivity of 1.7 Ohm-m) is still sometimes used as an electrical conductivity standard for metals. The best method to increase the electrical and thermal conductivity of copper has been to decrease the impurity content. The existence of impurities and all common alloying elements, except for silver, will decrease the electrical and thermal conductivity of copper [57 and 58]. As the amount of the second element increases, the electrical conductivity of the alloy decreases. Cadmium has the least detrimental effect on the resulting alloy's electrical conductivity, followed by increasing effects from zinc, tin, nickel, aluminum, manganese, silicon and phosphorus [57 and 58].

By considering the effect of alloying elements on the strength and the electrical conductivity of copper, it is comprehensible that minor additions of nickel would have a minimal detrimental effect on the electrical conductivity, especially when a small amount of silver is also added to the alloy. 


\section{MECHANICAL MILLING}

\subsection{Introduction}

Mechanical milling is an effective technique for the preparation of fine metallic powders. It is a solid state processing technique, where equilibrium and non-equilibrium phases are synthesized. The mechanical energy transferred to the powder particles in these mills takes place by shear action or impact of the high velocity balls with the powder. It is considered to be one of the most robust processing routes for synthesizing a variety of materials.

Milling devices include planetary machines, where ball motion is generally chaotic, and vibrational mills, which may use either rods or balls. Milled products include amorphous, nanocrystalline and quasicrystalline materials [47- 49]. During milling, solid-solid, solid-liquid and solid-gas reactions are initiated through repeated deformation and fracture of powder particles under a controlled atmosphere. The most noticeable change with milling is an initial increase in size of particles due to agglomeration [59] followed by a reduction in the particle size, generally to nanoscale depending on milling duration. In addition to size refinement, milling has received wide acceptability due to its potential to drive the changes at ambient conditions which are either not possible or require vigorous conditions in conventional processing routes [5052]. For example, while unfavorable decomposition steps restrict the high temperature chemical reactions of a number of materials, they can be easily brought about through milling at room temperature itself [53]. 


\subsection{Milling purposes}

Milling may serve the following purposes in engineering:

1. Increase in surface area of a solid

2. Manufacture of a solid with a desired particle size

3. Pulping of resources

Milling is employed mainly to produce fine particle rendered through attrition and compressive forces at the grain size level. The ball milling machine employed in the current research was the SPEX SamplePrep 8000M Mixer/Mill.

\subsection{SPEX SamplePrep Mixer/Mill}

The SPEX SamplePrep Mixer/Mill (also known as high-energy shaker mills) is a variant of the ball mill or jar mill, in which powders are ground in a container along with one or more grinding elements. It can be described as laboratory scale that is designed to pulverize a sample rapidly while mixing it homogeneously - an approach long known to be effective in preparing samples for emission spectroscopy and X-ray fluorescence (XRF) spectrometry. The SPEX SamplePrep 8000 Mixer/Mill is also widely used for blending powders and making emulsions and has recently become indispensable in the search for superconducting alloys [59].

The containers are usually cylindrical; the grinding elements are most often balls but may be rods, cylinders, or other shapes. Generally, the containers and grinding elements are made from the same material. As the container is rolled, swung, vibrated, or shaken, the inertia of the grinding elements causes them to move independently into each other and against the container wall, thus grinding the sample. Variations of the ball mill 
range from laboratory mills with a sample capacity of a gram or less to large industrial mills with a throughput of tons per minute.

The shaking action of a SPEX SamplePrep Mixer/Mill is both complex and forceful. As the vial is swung back and forth in a shallow arc, its ends are displaced laterally. This distributes the grinding-ball impact over wide areas of the caps. In typical ball mills there are many grinding elements which travel only a short distance before impact; in SPEX SamplePrep Mixer/Mill vials some of the balls travel the entire length of the vial at high velocity and high G-forces.

A distinct advantage of the SPEX Sample Prep Mixer/Mill design is the flexibility of having a large selection of vial materials such as hardened steel, stainless steel, tungsten carbide, alumina ceramic, zirconia ceramic, silicon nitride, agate, and plastic. Not only do these different media offer a wide range of grinding and blending performance, but they reduce contamination problems by allowing the analyst to avoid materials whose presence in the ground sample is objectionable.

\subsection{Milling Methods}

The two common methods of milling are wet ball milling and dry ball milling. Occasionally, metal powders are milled with a liquid medium, which is referred to as wet grinding; in the absence of liquid it is referred to as dry grinding. However, wet grinding has a serious disadvantage in that it is accompanied by increased contamination of the powder. Thus, most milling operations have been conducted in the dry mode. 


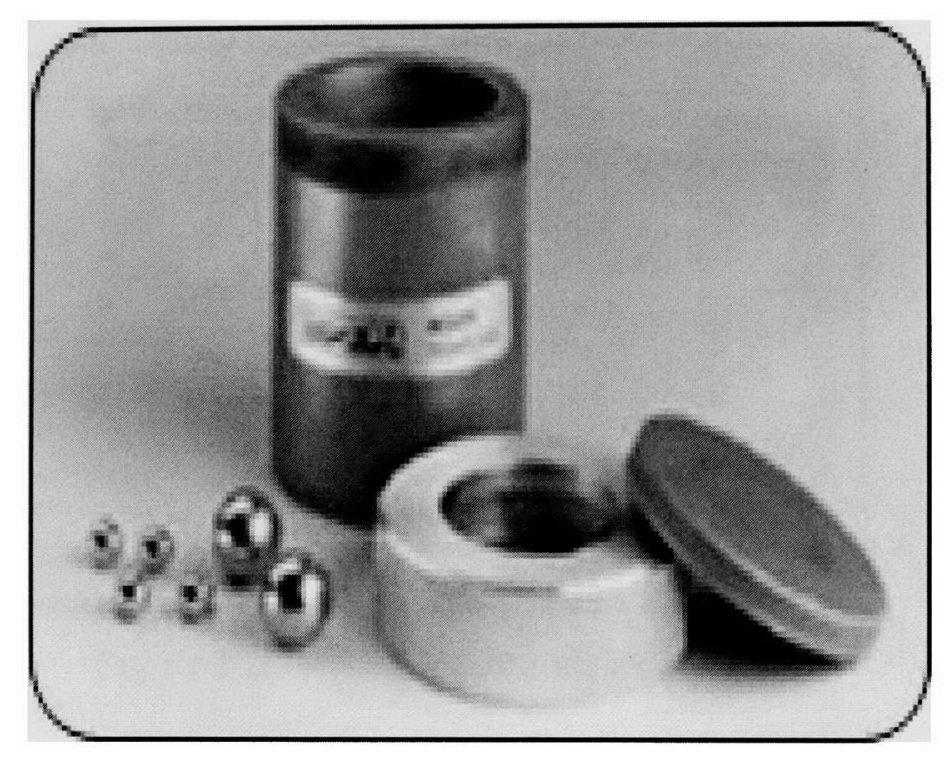

Figure 2.1: Hardened steel vial set with stainless steel ball set

\subsection{Milling Parameters}

\subsubsection{Milling Container}

In mechanical milling, the material of the container should be similar to that of the powder being milled in order to avoid contamination. On the other hand, if the milling container and the powder are that of same material, the chemistry may be altered by the addition of the element from the milling container to the powder. Additionally, the shape of the container also plays an important role. Mainly, there are two types of SPEX mill containers being used: flat-ended and round-ended containers; of which the flatended has proven to be more effective due to the occurrence of significantly higher rates of milling [52]. Over the years, the SPEX mill with a flat-ended hardened steel vial and stainless steels balls has been successfully employed [54 and 55]. A hardened steel vial with stainless steel ball set can be seen in Figure 2.1 and Figure 2.2. 


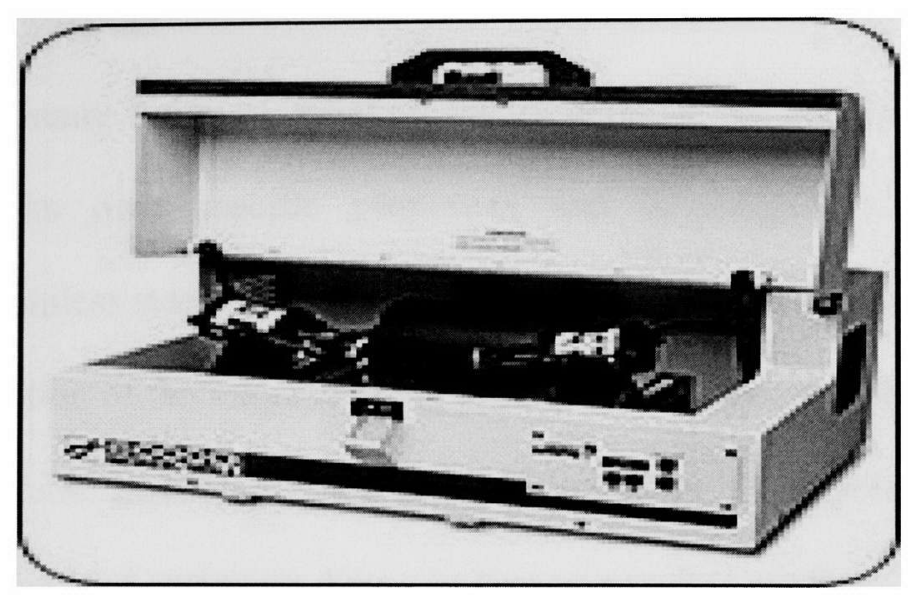

\subsubsection{Milling Speed}

Figure 2.2: $\quad$ SPEX 8000 Mixer / Mill

Generally the higher the speed, the higher is the energy imparted onto the powder. However, higher milling speeds usually result in an increase in temperature of the vial which could result in further contamination of the powders. The standard SPEX 8000 mill has a fixed milling speed of about 1200 rotations per minute (rpm).

In order to limit agglomeration and sticking effects in the current research, the powder was scraped from the vial surface at regular intervals. A milling time circuit of 10 minutes (milling) +20 minutes (idle time) was observed for every 10 minute of milling to minimize the increase in temperature during milling.

\subsubsection{Milling Time}

Milling time was considered to be the most important factor, for it mainly controls the desired particle size. Normally, the time is chosen so as to achieve a steady state in particle size reduction. Since all the milling parameters are interdependent, the milling time required depends on the type of the mill being used, intensity of the milling, charge ratio and milling temperature, etc. 


\subsubsection{Grinding Medium}

There are many types of grinding media suitable for use in a ball mill, each material having its own specific properties and advantages. Common in some applications are stainless steel balls. While usually very effective due to their high density and low contamination of the material being processed, stainless steel balls are unsuitable for some applications, including black powder and other flammable materials, which require non-sparking lead antimony, brass, or bronze grinding media.

\subsubsection{Charge Ratio}

The ratio of weight of balls to the powder being milled is termed the charge ratio (CR) or balls-to-powder ratio (BPR). Again, BPR is dependent on the type of mill being used. Usually a BPR of 10:1 is employed when a small capacity mill like the SPEX mill is being used, while the BPRs for other mills may be high as high as 100:1 depending on the capacity of the mills. The BPR has a significant effect on the milling time required to achieve a particular phase. Generally, the higher the BPR, the higher is the impact energy, and the shorter is the time required. But there is a disadvantageous possibility of an increase in temperature leading to the crystallization of the amorphous phase, thereby resulting in non-desirable phases. Additionally, an increase of impact energy tends to destroy MWCNTs, which could be detrimental to the intended property to be imparted to the composite. In the current research, a low BPR and a short milling time were used for mixing the composite elements in order to ensure minimal destruction of MWCNTs. It should be noted that MWCNTs were used in this investigation, such that if some damage occurred to the MWCNTs, it would only be confined to the outer walls. In fact, SEM analysis (see the photomicrographs in Fig 7.7 (a), (b) and 7.8 (a), (b)) of unfired pellets 
revealed that the diameter of the MWCNTs were $20-25 \mathrm{~nm}$ as compared to the as received MWCNT diameters of $10-20 \mathrm{~nm}$

\subsubsection{Milling Atmosphere}

Selection and maintenance of a suitable milling atmosphere is of major importance since it has a direct impact on the contamination of the powder. Again, its selection depends on the end products desired. But, when highly reactive powders are to be milled, the atmosphere should be strictly maintained inert. An argon atmosphere was used in this research. Generally, the loading and unloading of the powders were performed inside an atmosphere-controlled glove box, which was evacuated with a vacuum pump and purged with argon.

It should be noted that all of the aforementioned process variables are not completely independent, because the optimum milling time depends on the type of mill, size of the grinding medium, ball-to-powder ratio, etc. Therefore, in order to achieve the optimum milling conditions, preliminary tests had to be conducted by varying each parameter until the desired product was obtained.

\subsection{Powder Handling}

Milling involves working with powders of very small particle sizes, which require handling with caution and care. Because of the inherent large surface area, small particles are highly reactive, pyrophoric and can cause health problems when inhaled. Thus, all precautions were taken not to open the powder to the atmosphere at any time in order prevent oxidation or ignition. In this investigation, the milled powders were always 
removed in a glove box under argon and allowing the vial to cool down to the room temperature, before being stored in airtight glass containers.

\subsection{Powder Contamination}

Contamination of the powders being milled is the most serious concern in the process of mechanical milling. Fineness of the powder, milling atmosphere, material type of milling equipment, temperature increase during milling etc., are some of the factors that contribute to contamination during a milling process.. Since fine powders possess high surface energy, they are highly reactive. Hence, proper care should be taken in handling the powder. Not maintaining a proper milling atmosphere was found to be the main contributor to contamination. A milling container that is not properly sealed is prone to leakage and could result in oxidation as well as contamination of the powdered material. Furthermore, the need of maintaining inert or suitable milling atmospheres is more emphasized by the fact that fresh surfaces formed due to collisions during the milling process are highly reactive. Also, to minimize the contamination, it is always essential to use milling equipment (vial and balls) whose hardness is greater than that of the powder being milled. Additionally, a temperature increase during the milling process also increases the likelihood of contamination. Nevertheless, metal powder coats the inner walls of the vial and the grinding medium during the initial stages of milling and helps to reduce the contamination. However, precautions should be taken so that the chemistry of the powder mixture is not compromised. Thus, contamination, unless special measures are taken to minimize/avoid it, seems to be an inherent drawback of the mechanical milling technique. 


\subsection{Cleaning Containers}

Grinding containers were cleaned between sample runs in order to avoid crosscontamination, and the procedure can be as simple or as complex as the analytical objectives warrant. In some applications simple wipe down with ethanol was adequate; another practical approach was to brush out the container, then briefly grind an expendable portion of the next sample, which was discarded.

For more thorough cleaning one may grind one or more batches of pure quartz sand, and then wash the container thoroughly. In extreme cases, such as the plating of container walls with a malleable metal, chemical cleaning or multiple grinds with quartz may be necessary. An effective single-step grinding procedure for most grinding containers is to grind pure quartz sand together with hot water and detergent, then rinse and dry the container. Drying is accelerated by the use of blow-dryer or similar appliances. A safety advantage of this cleaning method is that it controls respirable airborne dust.

A cleaning procedure is easily evaluated by grinding and analyzing a known sample, or by checking the impurities appearing in successive batches of ground quartz sand. It should be noted that grinding containers become more difficult to clean with age because of progressive pitting and scratching of the grinding surfaces.

Hardened steel containers can rust. While iron oxide coatings can be removed by warm dilute oxalic acid solution or abrasive cleaning, it was recommended that steel containers should be thoroughly dried after cleaning and, if stored, kept in a plastic bag with a desiccating agent. 


\subsection{Consolidation}

In any powder metallurgical process, consolidation of the milled product is a very important phase and efficient methods of consolidating powders into bulk shapes are required. Almost every consolidation method employed in powder metallurgy processes can also be applied to milled powders. However, it should be realized that bulk consolidation should be accomplished without losing the benefits of metastable effects or nanostructured phases developed by mechanical milling. In industry, the mechanically milled powders are generally consolidated through Hot Isostatic Pressing (HIP), powder rolling, Ceracon processing, plasma activated sintering, electro-discharge pelletion, and explosive methods (shock method).

Since mechanically milled powders possess high hardness due to work-hardening, cold pelletion is not a very suitable consolidation approach. However, the purpose of this investigation was to manufacture a $\mathrm{Cu}-\mathrm{MWCNT}$ composite and measure its electrical conductivity. Thus this technique of consolidation was employed in this research. Cold pelletion is a simple but effective densification method that can produce pellets with relatively high densities. Due to the increased hardness of the mechanically milled powders, a high deformation pressure with ample holding time was required for the cold pelletion of pellets. Figure 2.3 illustrates the process of uniaxial cold pelletion, where the powder, is loaded in the bore of the die, and it is pelleted using a punch by applying pressure uniaxially. The density of the pellet obtained depends mainly on particle size of the powder, which influences its packing density, and plasticity.. Lu et al [56] proposed that the pelletion process could be divided mainly into three stages: 
- Particle rearrangement, wherein the particle rearrange themselves in the bore, when pressure is applied, without any deformation.

- Deformation, wherein the actual elastic and plastic deformation takes place under the applied pressure.

- Work hardening and fragmentation, which in the case of pelletion of milled powders has little influence since the powders are already work-hardened.

It is anticipated that application of novel and innovative consolidation methods, mentioned in the previous paragraph, to the mechanically milled $\mathrm{Cu}-\mathrm{MWCNT}$ powders would open new frontiers for the development of metal-MWCNT composites in the future.

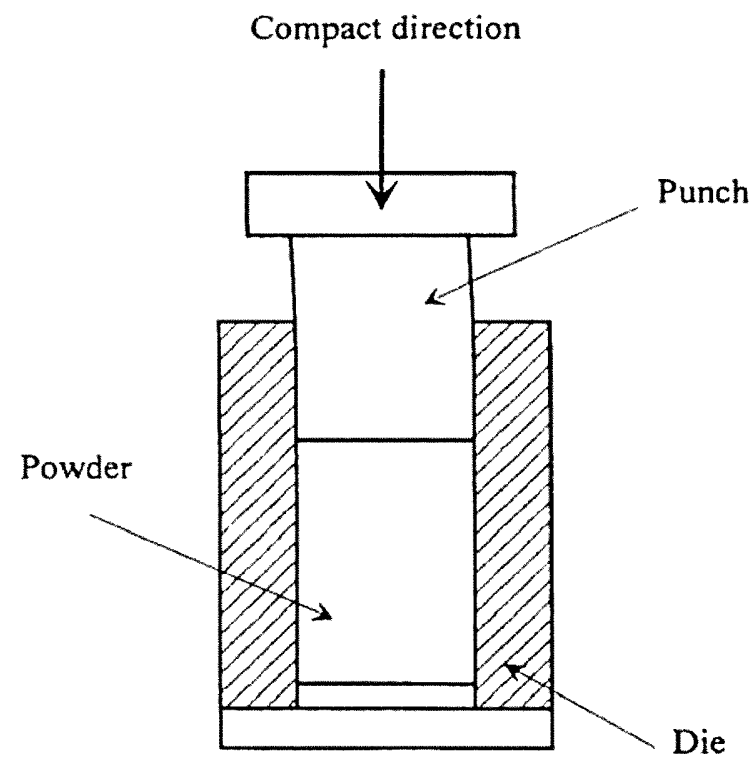

Figure 2.3: Uniaxial cold pelletion 


\section{POROSITY}

\subsection{Introduction}

The porosity of a material is defined as the total proportion of the air space contained between the solid particles of which the body is composed. Any material body is made up of particles of different types and sizes. The space between particles is called pore space. Pore space determines the amount of water that a given volume of pellet can hold.

No matter how carefully the various size particles of a raw material are mixed and pelleted, it is virtually impossible to produce a body free from void space. All materials with the exception of glasses and similar bodies are to some extent porous. The porosity of a material has a great influence on many properties, such as absorption, resistance, conductivity, strength etc. In the case of composites, the electrical conductivity decreases as the porosity increases [62]. Since the objective of this research was to produce a $\mathrm{Cu}$ composite of high electrical conductivity, the determination of porosity of pellets is of paramount importance.

\subsection{Different Kinds of Porosity}

Pores produce two sets of results when the porosity of a material is determined, viz (a) the true porosity, and (b) the apparent porosity [62]. The true porosity is the ratio between the volume of voids, both open and closed pores, and the total volume of the article. The apparent porosity is defined as the ratio between the volume of water or liquid capable of being absorbed into it and the total volume of the article. Any closed pores, or for that matter, any open ones which are so fine that liquid cannot penetrate into 
them, will not be included as void space in determining the apparent porosity. Both the true and apparent porosity may be expressed as

(a) Percentage by weight

(b) Percentage by volume

The percentage of porosity by weight shows the weight of water or other liquid absorbed by 100 units of weight, whilst the percentage of porosity by volume is the volume of water absorbed in 100 volumes of the material or article. It is customary to use the latter as this indicates the volume of the pores. The volume of the articles of the simple shape may be calculated from direct measurement of the dimensions, but where the shape is complex the volume must be determined by finding the difference between the weight when immersed in water and weight in air. The expression 'porosity by weight' is unsatisfactory because all the materials do not have the same density, so that the 'porosity' of different samples cannot be compared accurately. The expression of porosity wholly in terms of volume is, therefore, the most satisfactory as well as the most consistent measurement.

\subsection{Parameters Influencing Porosity}

The porosity of various materials may be influenced by some or all of the following [62]:

(a) The shape of the particles

(b) The size of the particles

(c) The grading of the particles

(d) The nature of the materials comprising the mixture 
(e) The treatment to which the materials are subjected during manufacture and

(f) The relative position of the particle, i.e. whether they are closely pelleted or lie loosely on one another.

Porosity can be reduced by carefully grading although in general, the coarser the maximum size of the particles the less will be the porosity; in other words an ideally well-graded mixture of fine particles does not approach the theoretically-predicted minimum porosity figure as closely as a coarser mixture. A mixture of angular grains readily pellets under pressure to give a low porosity. A fact which is often overlooked is that if the individual grains in a body are themselves porous, the body itself will have porosity higher than that of similarly graded mixture of non-porous particles. The porosity is also greatly influenced by the methods used for molding and the reactions which occur on firing.

\subsection{Porosity Measurement}

As explained previously there is a distinction between the true and apparent porosity, but in most cases the term porosity is applied to the apparent porosity as estimated from the amount of water or other suitable fluid absorbed by a given weight or volume of the sample. Hence the absorption or apparent porosity is a measure of the unsealed pores.

The usual way of carrying out the determination [62] is to take conveniently sized test piece, which is weighed $\left(\mathrm{W}_{1}\right)$ after being thoroughly dried. To ensure that liquid completely fills all unsealed pores the sample should be placed in a vacuum vessel, which is evacuated. Without releasing the pressure, the immersed liquid must then be introduced 
to such a level that the piece is entirely covered. The saturated sample may then be weighed in air $\left(\mathrm{W}_{2}\right)$ by suspending it from a thread to the arm of the balance; and finally the weight of the sample when immersed in water $\left(\mathrm{W}_{3}\right)$ has to be measured.

The weight of the liquid absorbed is given by $\mathrm{W}_{2}-\mathrm{W}_{1}$ gram, provided that water (density $=1$ ) is used for immersion. The total volume of the test piece is $W_{2}-W_{3}$, i.e. the weight 'lost' on immersion in water, so the percentage apparent porosity by volume $(\mathrm{P})$ is given by the equation:

$$
P=\frac{W_{2}-W_{1}}{\left(W_{2}-W_{3}\right) \rho}
$$

where $\rho$ is the density of the liquid which will be 1 gram per cubic centimeter $\left(\mathrm{gms} / \mathrm{cm}^{3}\right)$ if water is used. 


\section{ELECTRICAL CONDUCTIVITY}

\subsection{Introduction}

Electrical conductivity or specific conductivity is a measure of a material's ability to conduct an electric current. Conductivity is the reciprocal (inverse) of electrical resistivity and has the SI units of siemens per meter $\left(\mathrm{S} \cdot \mathrm{m}^{-1}\right)$ i.e. if the electrical conductance between opposite faces of a 1 meter cube of material is 1 siemens then the material's electrical conductivity is 1 siemens per meter. Electrical conductivity is commonly represented by the Greek letter $\sigma$, but $\kappa$ or $\gamma$ are also occasionally used.

\subsection{Effect of Temperature on Electrical Conductivity}

Electrical conductivity is strongly dependent on temperature. In metals, electrical conductivity decreases with increasing temperature, whereas in semiconductors, electrical conductivity increases with increasing temperature. Over a limited temperature range, the electrical conductivity can be approximated as being directly proportional to temperature. In order to compare electrical conductivity measurements at different temperatures, they need to be standardized to a common temperature. This dependence is often expressed as the slope of the conductivity-vs-temperature graph, and can be expressed as follows:

$$
\sigma_{T^{\prime}}=\frac{\sigma_{T^{\prime}}}{1+\alpha\left(T-T^{\prime}\right)}
$$

where,

$\sigma \mathrm{T}^{\prime}$ is the electrical conductivity at a common temperature, $\mathrm{T}^{\prime}$ $\sigma \mathrm{T}$ is the electrical conductivity at a measured temperature, $\mathrm{T}$ 
$\alpha$ is the temperature compensation slope of the material

$\mathrm{T}$ is the measured absolute temperature

$\mathrm{T}^{\prime}$ is the common temperature

\subsection{Effect of Porosity on Electrical Conductivity}

Porosity introduces in-homogeneity in the bulk material, which decreases its electrical conductivity. The presence of pores in a solid not only significantly degrades the electrical characteristics but also the decreases the strength of the material. The porosity in turn is also dependent on milling parameters such as milling time, BPR, size of the grinding medium and the method of pressing/consolidation. Thus, it is evident that milling parameters not only influence the porosity of the composite but indirectly influence its electrical conductivity as well. For these reasons, great care was taken in determining the milling parameters in this investigation.

\subsection{Electrical Resistivity Measurement}

A HP 4263A LCR meter (Figure 4.1) was used to measure the sheet resistance of pellets prepared. It uses the Four - Point probe measurement configuration, which permits easy, stable, and accurate measurements of resistance and avoids the measurement limitations due to factors such as mutual inductance, interference of the measurement signals, and unwanted residual in the test connections.

A schematic representation of the Four-Point probe is shown in Figure 4.2. It consists of four coaxial connectors:

1. $+\mathrm{I}: \mathrm{H}_{\mathrm{CUR}}:$ High Current

2. $+\mathrm{V}: \mathrm{H}_{\mathrm{POT}}:$ High Potential 
3. $-\mathrm{V}: \mathrm{L}_{\mathrm{POT}}$ : Low Potential

4. -I : $\mathrm{L}_{\mathrm{CUR}}$ : Low Current

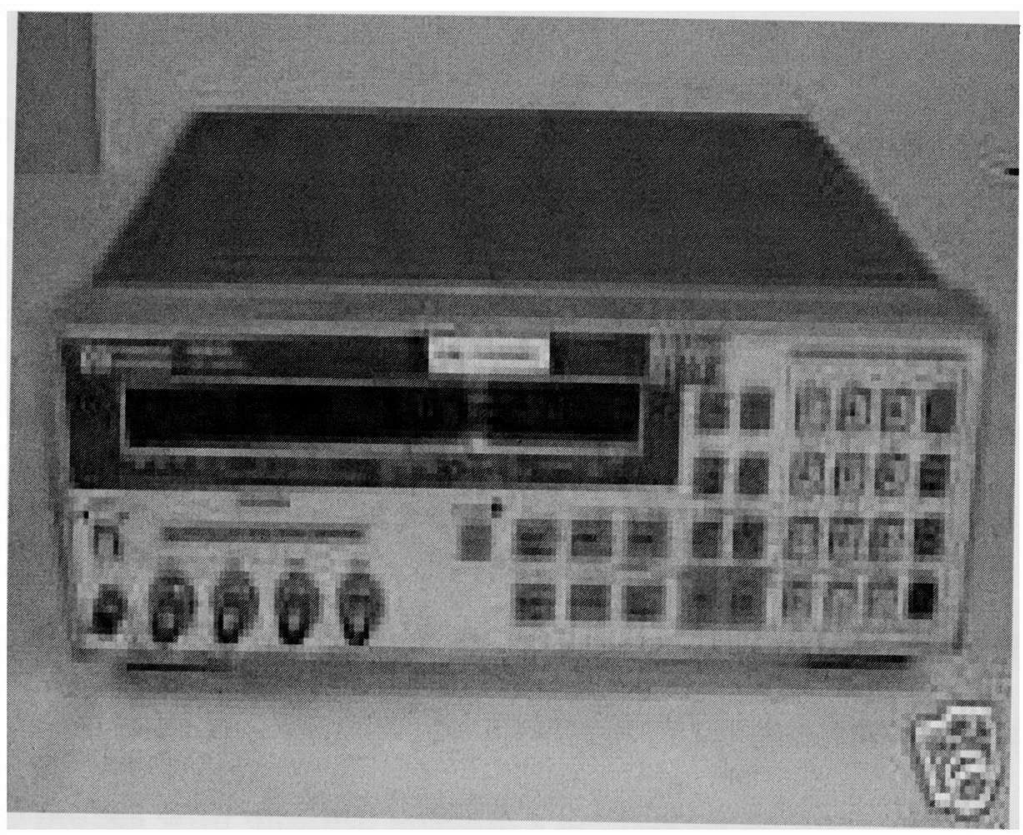

Figure 4.1: $\quad$ Four probe HP 4263A LCR meter

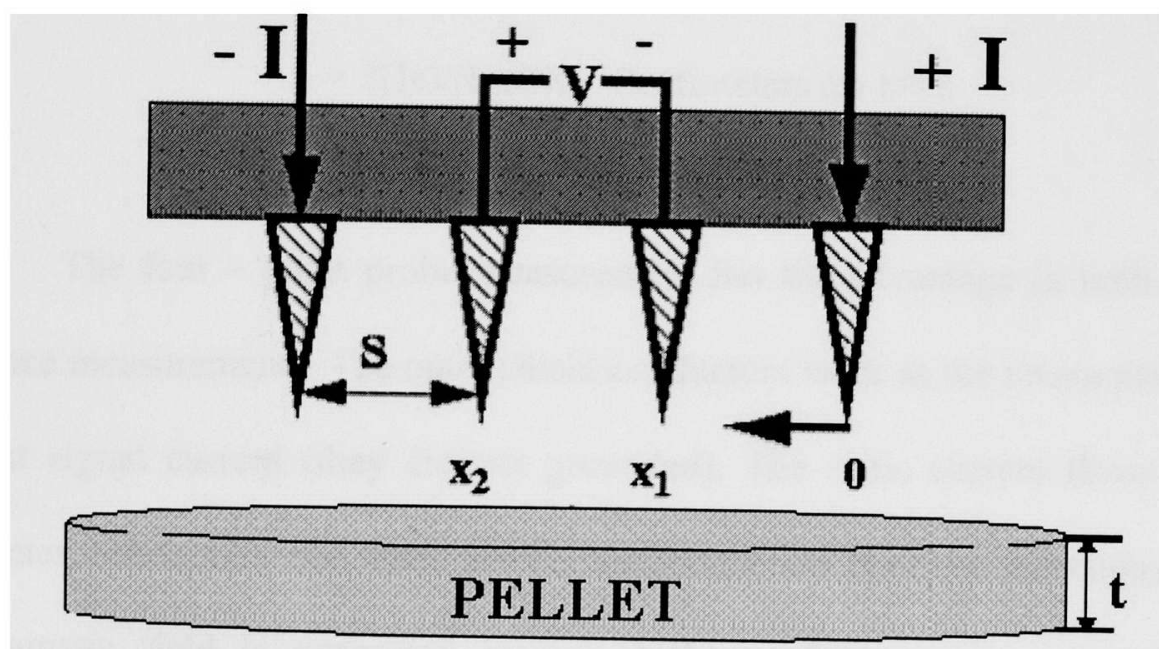

Figure 4.2: Schematic representation of the Four-point probe 
The resistivity, $\rho$ is independent of specimen geometry but related to the resistance, $\mathrm{R}$ through the expression:

$$
\rho=\mathrm{RA} / 1
$$

Where $\mathrm{l}$ (or $\mathrm{t}$ ) is the thickness of the pellet and A (or s) is the distance between the probes. From the expression of Ohm's law, the resitivity is expressed in terms of the the voltage between probes $+\mathrm{V}$ and $-\mathrm{V}$ and the current that is measured between probes $+\mathrm{I}$ and $-\mathrm{I}$. The electrical conductivity, which is used to specify the electrical character of the composite, is simply the reciprocal of the resistivity.

The theory behind this method is based on a fixed current (I) that is injected into the material through the outer two probes, and a voltage $(\mathrm{V})$ is measured between the two inner probes. If the probes with uniform spacing $s$ are placed on an infinite slab material of thickness $t$, then the resistivity $\rho$, is given by:

$$
\begin{gathered}
\rho=2 \Pi s \mathrm{~s} / \mathrm{I} \mu \mathrm{Ohm}-\text { Centimeters for } \mathrm{t} \gg \mathrm{s} \\
\rho=(\Pi \mathrm{t} / \mathrm{ln} 2) \mathrm{V} / \mathrm{I} \mu \mathrm{Ohm}-\text { Centimeters for } \mathrm{s}>\mathrm{t}
\end{gathered}
$$

The four - point probe measurement has the advantage in both low and high resistance measurements. The outer shield conductors work as the return path for the measurement signal current (they are not grounded). The same current flows through both the center conductors and outer shield conductors (in opposite directions), so no external magnetic field is generated around shield conductors (the magnetic fields produced by the inner and outer currents completely cancel each other). Therefore, test leads do not contribute additional errors due to self or mutual inductance between the individual leads. 


\subsection{Limits of Measurement Capability}

The probes must be able to make ohmic contact with the material. Indium can also be used for a good ohmic contact. Very low resistive materials (e.g. aluminum, gold, platinum) require the maximum current from the current source to achieve a reading on the display. The current through the probe is restricted to $10 \mathrm{~mA}$ because of heating effects and excessive current density at the probe tips. An unclean sample or a sample that has surface doping will lead to inaccurate graphs due to an impeded ohmic contact or current leakage. 


\section{PRELIMINARY EXPERIMENTAL RESULTS}

\subsection{Selection of Milling Time of Composite Materials}

Since the electrical conductivity of the composite is dependent on the porosity which is interdependent on milling time, it was mandatory to determine effect of milling time on particle size. During the ball milling process, the metal powder initially formed agglomerates of large particles. In the early stages of milling, the particles are soft and their tendency to weld together and form large particles is high. Furthermore, the new surfaces created enable the particles to weld together which leads to an increase in particle size. After further milling, the particle size of these agglomerates decreased as a result of collision forces between the steel balls, which plastically deform the powder particles leading to work hardening and fracture. As a result, a broad range of particle sizes develops, with some as large as three times bigger than the starting particles.

With continued deformation, the particles become work hardened and fracture by a fatigue failure mechanism and/or by the fragmentation of fragile flakes. Fragments generated by this mechanism may continue to be reduced in size in the absence of strong agglomerating forces. After milling for a certain length of time, steady-state equilibrium is attained when a balance is achieved between the rate of welding, which tends to increase the average particle size, and the rate of fracturing, which also tend to decrease the average composite particle size.

In order to check the variation of copper particles size by ball milling, copper powder was ball milled at various intervals of time and a JEOL Scanning Electron Microscope (SEM) was used to determine the particle size distribution and layer 
thickness variation with milling time. $\mathrm{Cu}$ powder (1-5 microns as supplied) was ball milled with a BPR of 10:1 using large balls of $1 / 2$ inch diameter, which were preferred to ensure effective ball milling of the copper. Figure 5.1 shows SEM images of particles taken at various milling time intervals.

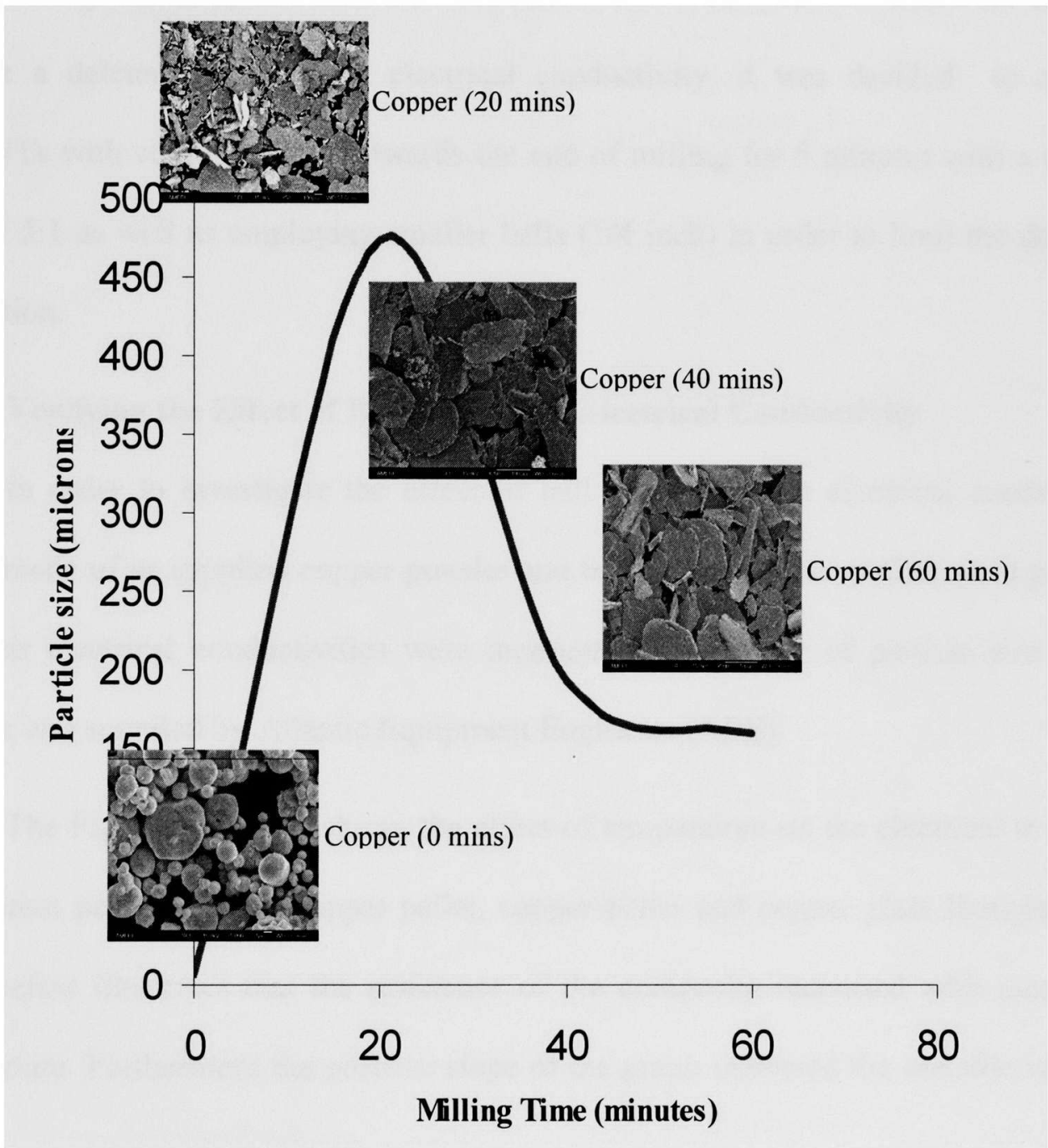

Figure 5.1: Change in particle size with milling time

From the SEM images and the graph depicted in Figure 5.1 the particle size of the milled powder remained relatively constant after 60 minutes of milling. Therefore, it was 
proposed to ball mill copper for at least 60 minutes to ensure that the agglomerated particles decrease in size.

Similarly MWCNTs were also ball milled for various intervals of time. It was found from the SEM images that MWCNTs were destroyed even when ball milled for 5 minutes using a BPR of 10:1 and 1/2 inch balls. Since destroyed MWCNTs are expected to have a deleterious effect on electrical conductivity, it was decided to mix the MWCNTs with copper powder towards the end of milling for 5 minutes with a reduced BPR of 5:1 as well as employing smaller balls (1/4 inch) in order to limit the degree of destruction.

\subsection{Verifying the Effect of Ball Milling on Electrical Conductivity}

In order to investigate the effect of ball milling on the electrical conductivity, pellets made of as supplied copper powder and ball milled copper pellets were prepared and their electrical conductivities were measured. The copper of particle size of 1-5 microns was supplied by Atlantic Equipment Engineers (AEE).

The Figure 5.2 below shows the effect of temperature on the electrical resistance of different pellets (milled copper pellet, copper pellet and copper plate (bought)). The graph below illustrates that the resistance of the composite increased with increase in temperature. Furthermore the positive slope of the graph indicated the metallic nature of copper.

The electrical resistivity and conductivity of the copper pellets that were prepared by pressing the copper powder (as supplied), milled copper powder and that of a copper plate that was purchased are compared below. There was no significant difference in 
electrical conductivity. However, a small decrease in electrical conductivity of the as supplied copper pellet may be attributed to its porosity, which as was previously discussed in Section 3.3, can be influenced by the particle size of the powder.

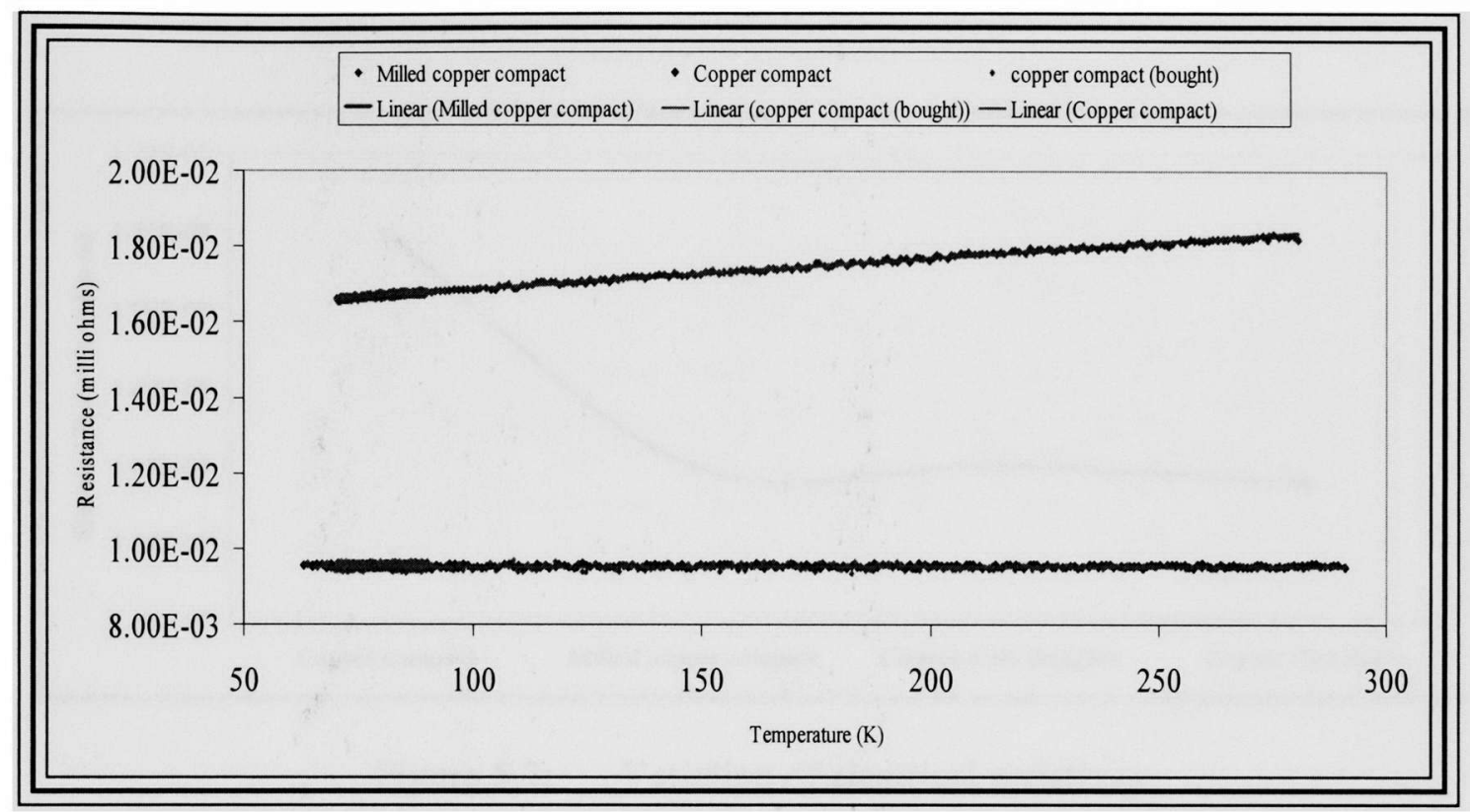

Figure 5.2: Resistance Vs temperature

Table 5.1: Comparison of electrical resistivity and conductivity

\begin{tabular}{|c|c|c|}
\hline Condition & $\begin{array}{c}\text { Resistivity (ohm.m) } \\
\text { (NTP) }\end{array}$ & $\begin{array}{c}\text { Conductivity (S.m } \\
\text { (NTP) }\end{array}$ \\
\hline Copper pellet & $1.71 \mathrm{E}-08$ & $5.85 \mathrm{E}+07$ \\
\hline Milled copper pellet & $1.68 \mathrm{E}-08$ & $5.94 \mathrm{E}+07$ \\
\hline Copper plate (bought) & $1.68 \mathrm{E}-08$ & $5.94 \mathrm{E}+07$ \\
\hline Copper (std ref*) [62] & $1.68 \mathrm{E}-08$ & $5.96 \mathrm{E}+07$ \\
\hline
\end{tabular}

The Figures 5.3 and 5.4 below illustrate the variation of electrical resistivity and electrical conductivity of copper by ball milling. From Figures 5.3 and 5.4 and Table 5.1, 
it is evident that ball milling had no detrimental effect on the electrical conductivity of copper. Moreover the electrical conductivity obtained for the copper plate (bought) and that of the standard reference [63] confirmed that the results from the four probe resistivity meter were accurate.

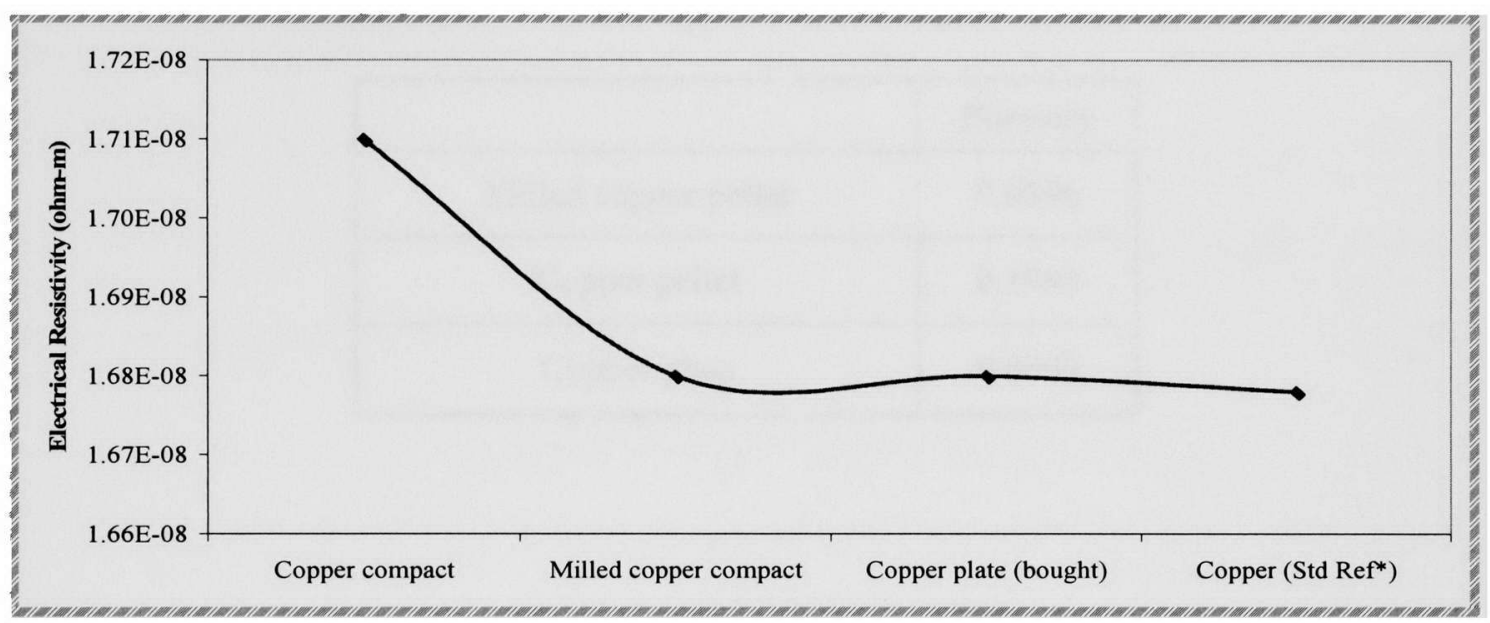

Figure 5.3: Variation of electrical resistivity

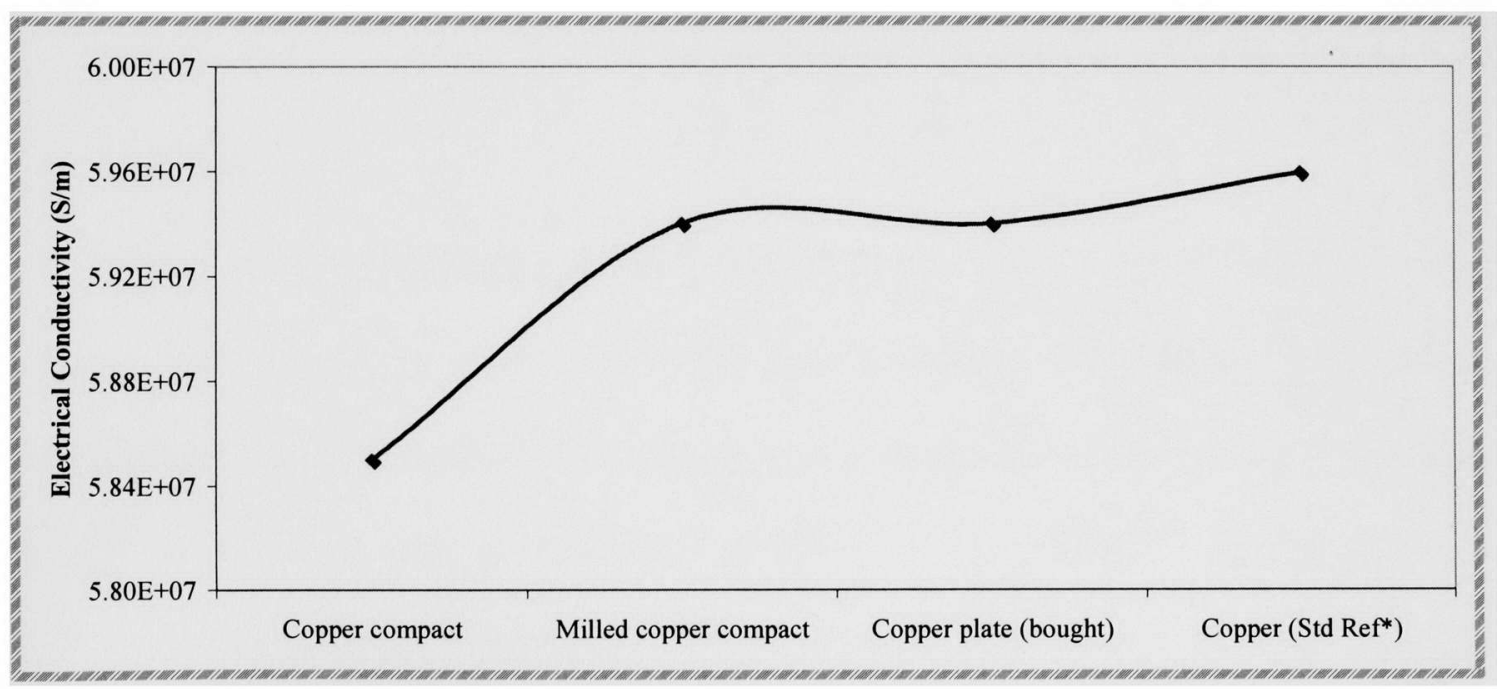

Figure 5.4: Variation of electrical conductivity

The Table 5.2 compares the porosity of milled copper pellets and as supplied copper pellets $(20,000 \mathrm{psi})$ with that of a pure copper plate (bought). The porosity values 
were consistent with the electrical conductivity measurements, in that the pure copper plate (lowest porosity) had the highest conductivity. The conductivity of the milled copper pellet was higher than that of the as supplied copper pellet, which possessed a lower porosity.

Table 5.2: $\quad$ Porosity of various copper materials

\begin{tabular}{|c|c|}
\hline & Porosity \\
\hline Milled copper pellet & 0.0896 \\
\hline Copper pellet & 0.1983 \\
\hline Copper plate & 0.0000 \\
\hline
\end{tabular}




\section{PREPARATION OF COPPER-CARBON NANOTUBE COMPOSITE}

\subsection{Mixing}

Pure copper metal powder ( $99.9 \%$ purity) composed of spherical particles (1-5 $\mu \mathrm{m}$ diameter) and Multi Wall Carbon Nanotubes (MWCNTs) (>95\% purity, $10-20 \mathrm{~nm}$ diameter) were mixed ( $4 \mathrm{~g}$ mixture) in a glove box under an inert gas atmosphere (Argon). Composites containing various compositions of MWCNT volume fractions 1:16, 1:12 and 1:8 were milled as described in Section 2.5, Table 6.1 shows the actual weights of each component present in the composite pellet.

Table 6.1: Composition of Cu-MWCNT composites studied

\begin{tabular}{|c|c|c|}
\hline Weight of Cu in grams & $\begin{array}{c}\text { Weight of MWCNT in } \\
\text { grams }\end{array}$ & Volume Fraction \\
\hline $3.8(95 \mathrm{wt} \%)$ & $0.2(5 \mathrm{wt} \%)$ & $1-16$ \\
\hline $3.867(97 \mathrm{wt} \%)$ & $0.133(3 \mathrm{wt} \%)$ & $1-12$ \\
\hline $3.912(98 \mathrm{wt} \%)$ & $0.086(2 \mathrm{wt} \%)$ & $1-8$ \\
\hline
\end{tabular}

\subsection{Milling}

High energy ball milling (HEBM) was conducted using a SPEX 8000 mill with a hardened steel vial ( $\varphi 21 / 4$ in $\times 3$ in) as the milling container and stainless steel balls as grinding medium. The powders were poured into the milling container while in the glove box, in an effort to trap argon in the container. A 10:1 BPR or charge ratio was maintained with grinding medium consisting of steel balls of two different sizes $(\varphi 1 / 2$ in and $\varphi 1 / 4$ in) for effective milling. The composite powders of different volume fractions were prepared by first milling copper alone, followed by milling both copper and MWCNT and using smaller balls as previously described in Section 2.5. 


\subsection{Pressing/Consolidation}

A stainless steel die was specially designed and manufactured for the consolidation of the milled, strained hardened powders into pellets. Using CARVER Evacuble Pellet Dies Two precision anvils were manufactured so that the pellet formed would be precise and devoid of cracks and imperfections. Milled powder (of about $0.5 \mathrm{~g}$ ) was carefully pored into the bore between the two anvils. Finally the powder was uniaxially cold pressed to a pressure of about $630 \mathrm{MPa}$ using a manual CARVER pellet press- 4555.L and held at that pressure for about 10 minutes to form the pellet. The pellet was then carefully ejected out of the die and stored in an airtight sample bag.

\subsection{Sintering}

Sintering of Cu-MWCNT pellet was conducted in a Lindberg $1200{ }^{\circ} \mathrm{C}$ High Temperature Tube Furnace, at $950{ }^{\circ} \mathrm{C}$, a temperature well below the melting point temperature of copper $\left(1030^{\circ} \mathrm{C}\right)$. The samples were sintered under an argon gas atmosphere to avoid oxidation with a ramp time of 30 minutes and holding time of 2.5 hours. The pellets were allowed to gradually cool down to room temperature.

\subsection{Electrical Conductivity}

The Four-Probe electrical resistivity meter (described in Section 4.4) was used to determine the electrical conductivity of the pellets before and after sintering to determine the effect of sintering. The electrical resistivity was first determined using the following formula:

$$
\rho=(\Pi t / \ln 2) \mathrm{V} / \mathrm{I} \mu \mathrm{Ohm} \text {-Centimeters for } \mathrm{s}>>\mathrm{t}
$$


The electrical conductivity was then calculated from the inverse of the electrical resistivity. .

\subsection{Porosity}

Porosity $(\mathrm{P})$ of the pellets was determined before and after the sintering using the procedure described in Section...., using the following formula

$$
P=\frac{W_{2}-W_{1}}{\left(W_{2}-W_{3}\right) \rho}
$$

where $\rho$, is density of the water, which is $1 \mathrm{~g} / \mathrm{cm}^{3}$.

Additionally, the porosity of re-sintered pellets were determined. Re-sintering of the pellets, which is known to heal micro cracks was conducted in the Lindberg $1200{ }^{\circ} \mathrm{C}$ High Temperature Tube Furnace, at $950^{\circ} \mathrm{C}$, under argon with the same heating regime. The porosity and electrical conductivity of re-sintered pellets are shown in Table 6.2.

Table 6.2: $\quad$ Process parameters and conductivity of re-sintered Cu-MWCNT composites

\begin{tabular}{|c|c|c|}
\hline & \multicolumn{2}{|c|}{ Volume Fraction } \\
\hline & 1:16 volume fraction & $1: 12$ volume fraction \\
\hline No Balls; (size, ins) & $5 ;(1 / 2 ")$ & $\begin{array}{c}5 ;(1 / 2 ") \text { - Cu milling; } \\
5 ;(1 / 4 ") \text { - MWCNT mixing }\end{array}$ \\
\hline Milling/Mixing time & 40 mins (Cu-MWCNT) & $\begin{array}{l}100 \text { mins }(\mathrm{Cu}) ; 10 \text { mins } \\
(\mathrm{MWCNT})\end{array}$ \\
\hline Pelletion Pressure, $\mathrm{MPa}$ & 630 for $10 \mathrm{~min}$ & 630 for $10 \mathrm{~min}$ \\
\hline Sintering Temperature, ${ }^{\circ} \mathrm{C}$ & 950 & 950 \\
\hline Conductivity before sintering, S-m & $1.38 \mathrm{E}+06$ & $1.68 \mathrm{E}+06$ \\
\hline Conductivity after sintering, $\mathrm{S}-\mathrm{m}^{-1}$ & $2.30 \mathrm{E}+06$ & $2.90 \mathrm{E}+06$ \\
\hline
\end{tabular}


As the pellet with 1:12 volume fraction possessed the highest conductivity, it was proposed to prepare future pellets by milling copper alone for 40 minutes followed by mixing with MWCNT's for 5 minutes. The results obtained are tabulated below in Table 6.3 .

Table 6.3: $\quad$ Process parameters and conductivity of $\mathrm{Cu}-\mathrm{MWCNT}$ composites

\begin{tabular}{|c|c|c|}
\hline & \multicolumn{2}{|c|}{ Volume Fraction } \\
\hline & $1: 12$ & $1: 8$ \\
\hline No Balls; (size) & $\begin{array}{c}5 ;(1 / 2 ") \text { - Cu milling } \\
5 ;(1 / 4 ") \text { - MWCNT mixing }\end{array}$ & $\begin{array}{c}5 ;(1 / 2 ") \text { - Cu milling } \\
5 ;(1 / 4 ") \text { - MWCNT mixing }\end{array}$ \\
\hline Milling/Mixing time & $\begin{array}{l}40 \text { mins }(\mathrm{Cu}) ; 5 \text { mins } \\
(\mathrm{MWCNT})\end{array}$ & $\begin{array}{l}40 \text { mins }(\mathrm{Cu}) ; 5 \text { mins } \\
(\mathrm{MWCNT})\end{array}$ \\
\hline Pelletion Pressure, MPa & $669.4(30 \mathrm{~min})$ & $669.4(30 \mathrm{~min})$ \\
\hline Sintering temperature, ${ }^{\circ} \mathrm{C}$ & 950 & 950 \\
\hline Conductivity, $\mathrm{S}-\mathrm{m}^{-1}$ & $5.32 \mathrm{E}+07$ & $1.06 \mathrm{E}+07$ \\
\hline
\end{tabular}

The re-sintered pellets, as previously mentioned were found to posses' higher electrical conductivity. This was due to a reduction in void space, which is essentially the porosity of the composite.

\subsection{Morphology of $\mathrm{Cu}-\mathrm{MWCNT}$ composites}

A JEOL Scanning Electron Microscope (SEM) equipped with Energy Dispersive Spectrometer (EDS) was used to determine the particle size and the MWCNT distribution in the pellet. SEM analysis was employed to analyze the phase distribution and composition of the pertinent phases of the milled powders and sintered pellets. All of the SEM analyses were conducted using secondary electrons. 
The SEM image of Figure 6.1 (a), (b) and (c) indicated some degree of bridging between the MWCNT's and the matrix of the composite material.

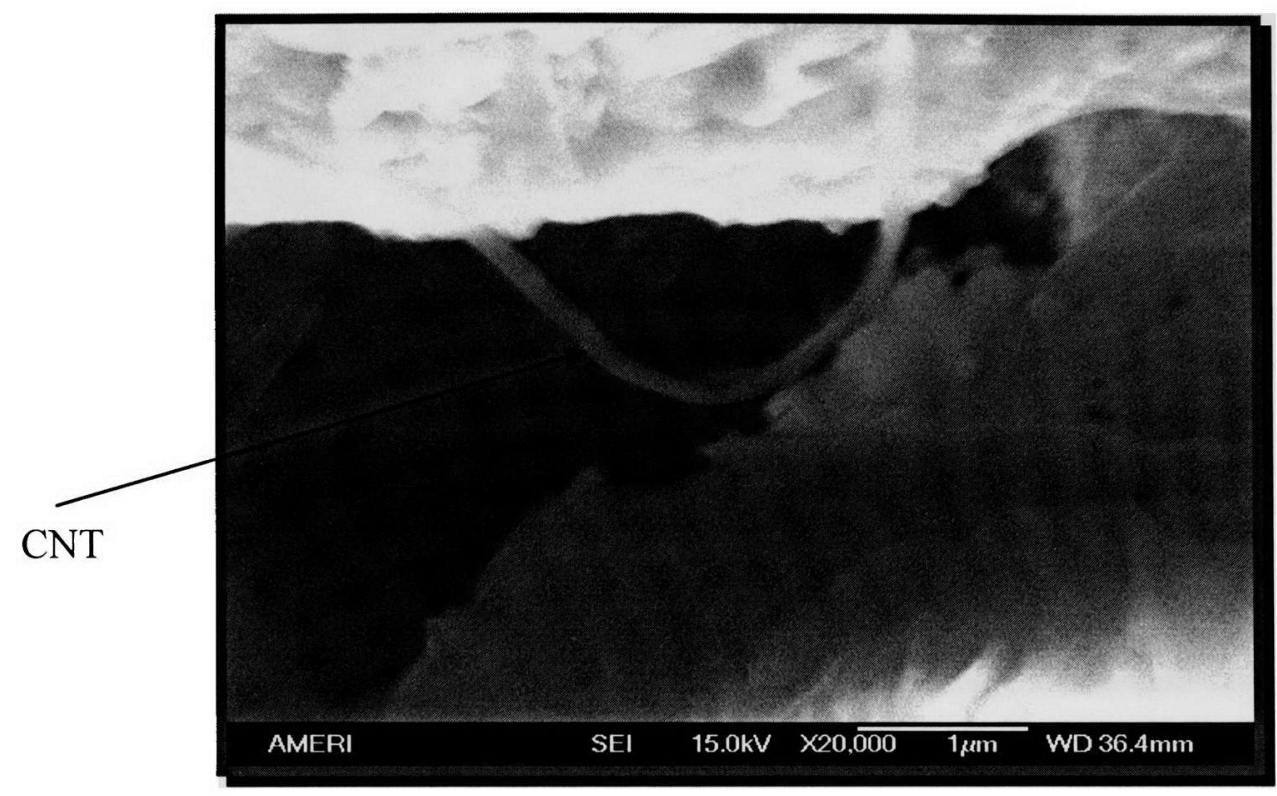

Figure 6.1 (a): SEM images of Cu-MWCNT composites

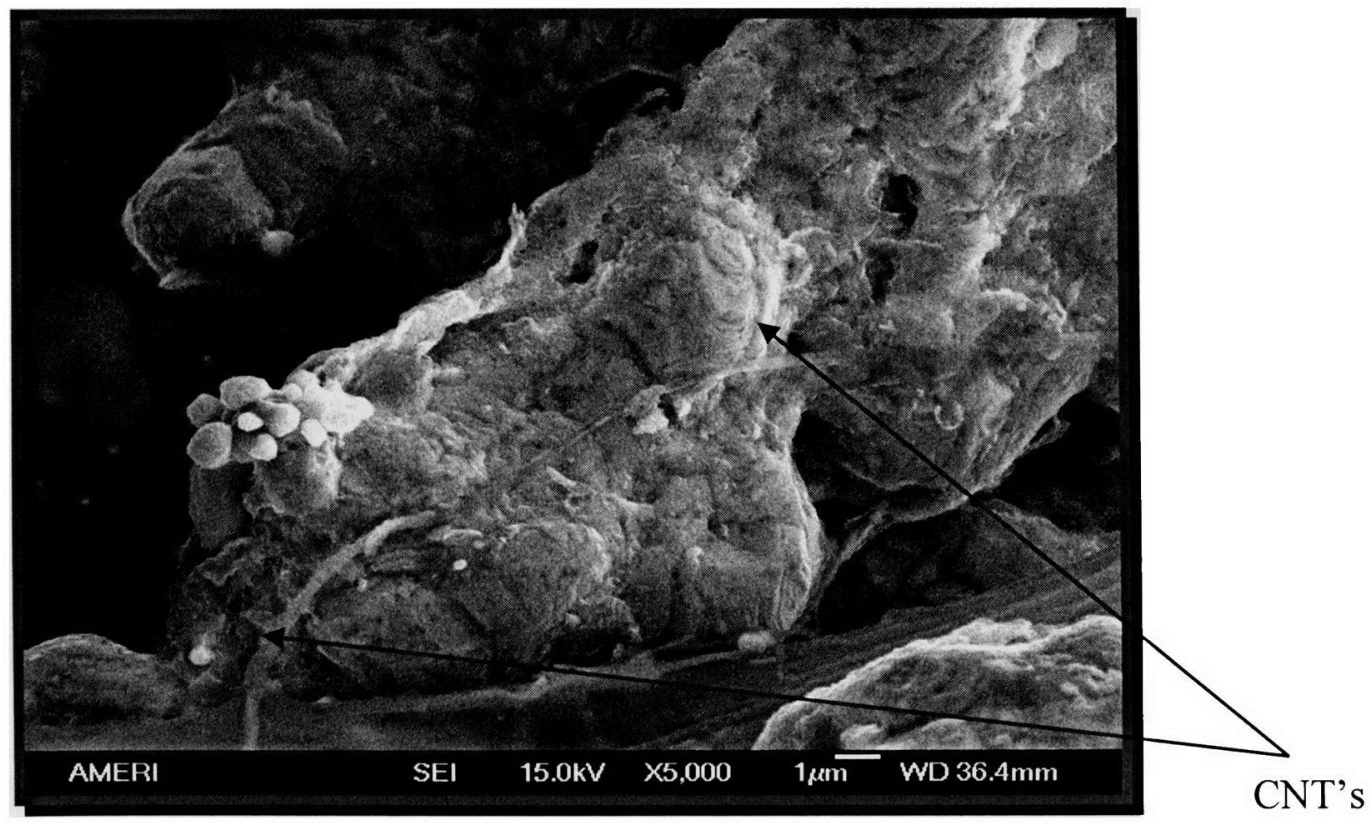


Figure 6.1 (b): SEM images of Cu-MWCNT composites

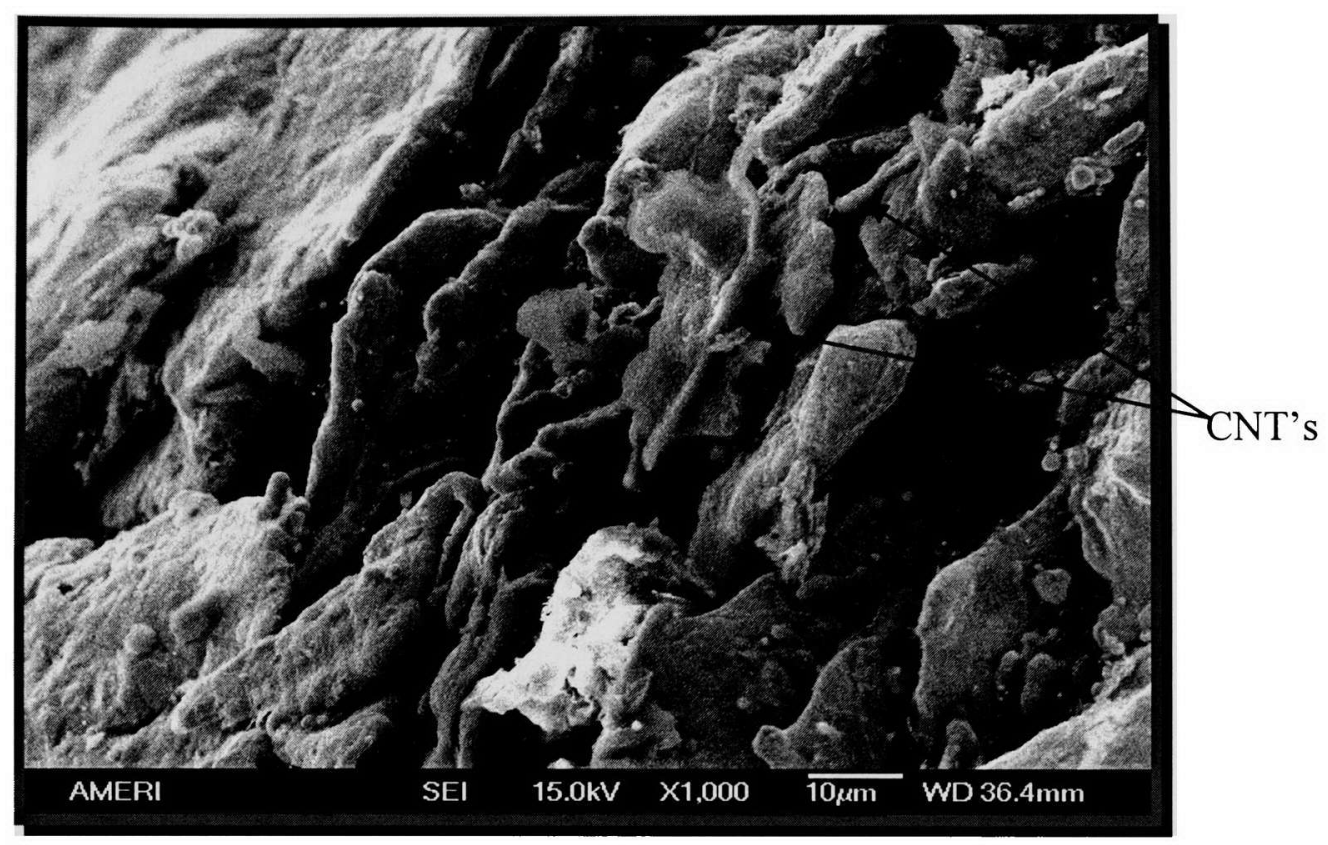

Figure 6.1 (c): SEM images of Cu-MWCNT composites

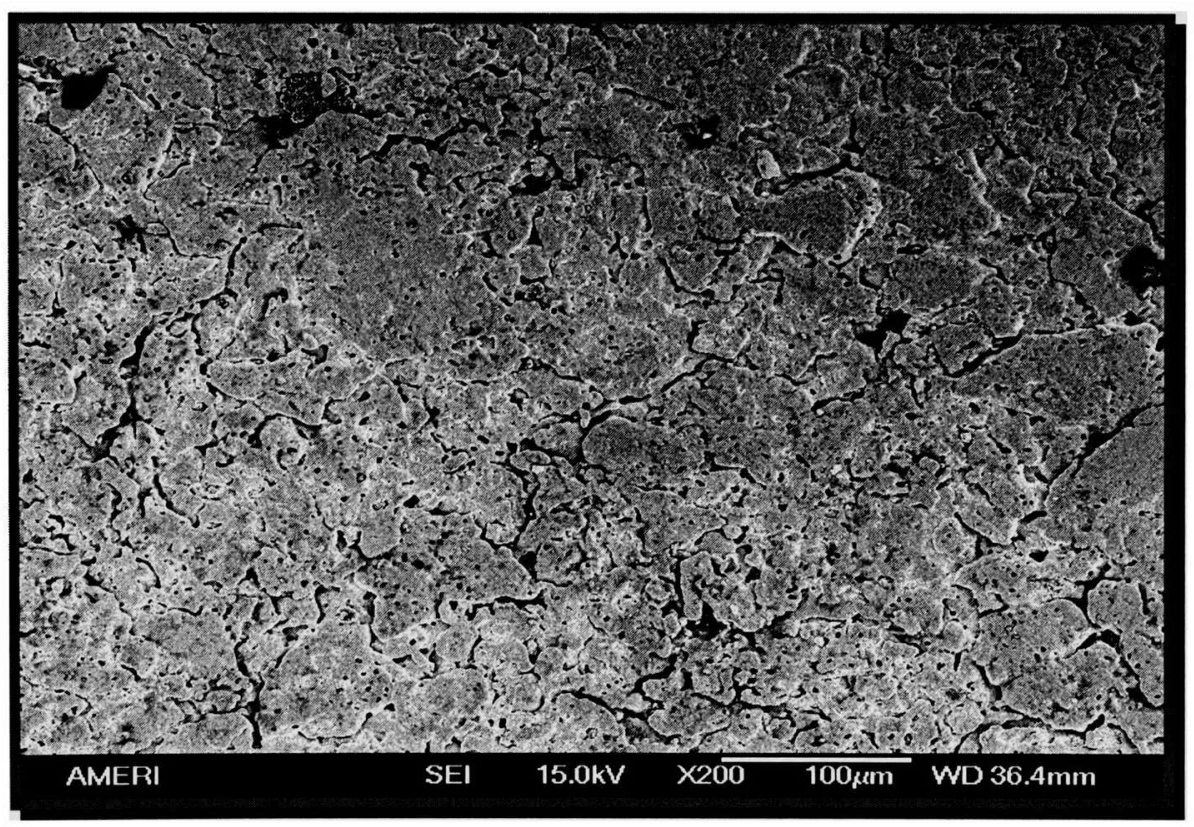

Figure 6.2 (a): Low magnification SEM morphology images of Cu-MWCNT composite 


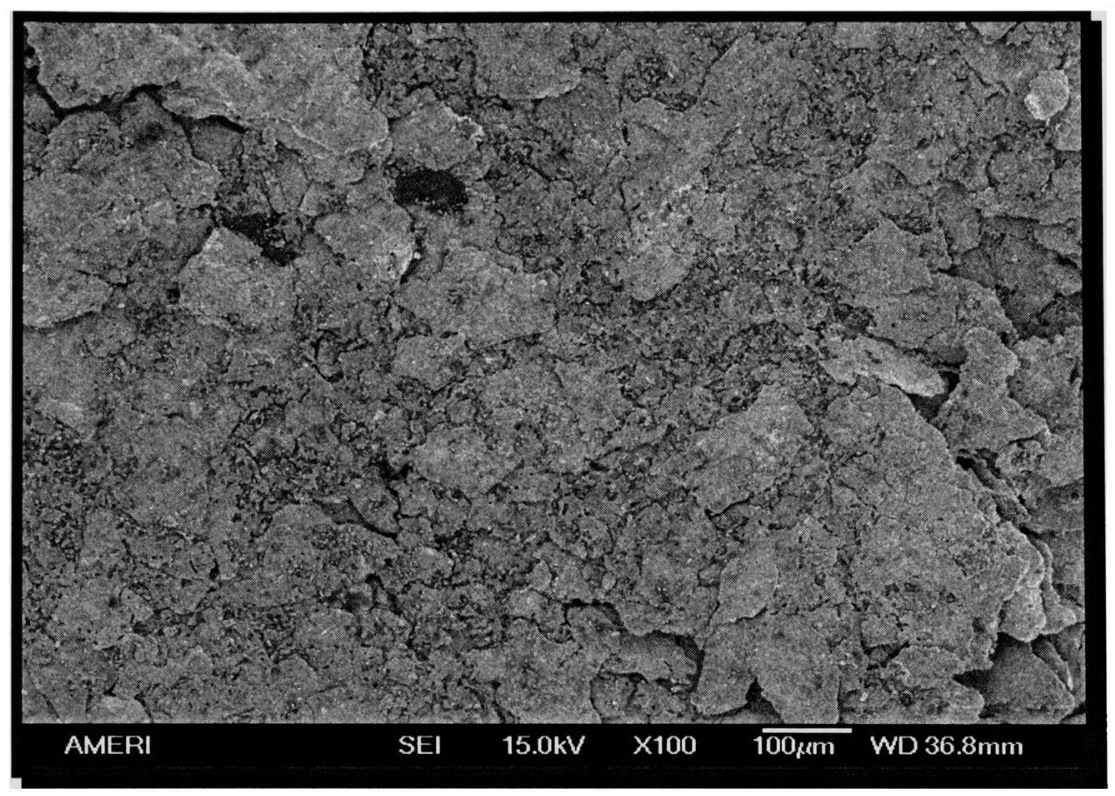

Figure 6.2 (b): Low magnification SEM morphology images of Cu-MWCNT composite

The low magnification SEM image in Figure 6.2 (a) and (b) illustrates the morphology of the Cu-MWCNT composite. The composite exhibited low porosity and displayed fracture dimples, which are indicative of a ductile material.

\subsection{Conclusions from preliminary experiments}

Based upon the preliminary experiments, it was observed that the pellets prepared by milling copper for 40 minutes and mixing MWCNT's for 5 minutes possessed higher electrical conductivities as shown in Tables 6.1 and 6.2. Therefore, it was decided that future pellets should be prepared by following the same procedure but to increase the milling time of copper to 100 minutes.

Small amounts of alloying elements are often added to metals to improve certain characteristics of the metal. In an attempt to improve poor interfacial bonding between $\mathrm{Cu}$ and MWCNT, as well as to increase the mechanical properties of the composite, $\mathrm{Ni}$ 
and $\mathrm{Ag}$ were added in the form of nickel and silver resinates. The small amount of $\mathrm{Ni}$ is not anticipated to have a serious detrimental effect on the electrical conductivity of cu as discussed in Section 1.4.2. The addition of $\mathrm{Ag}$ is expected to raise the softening temperature of $\mathrm{Cu}$ as well as improve its electrical conductivity [64]. Ag also improves the mechanical properties, especially the creep resistance. This type of material is therefore preferred where resistance to softening is required as for example in commutators or when the material is expected to sustain stresses for long times at elevated working temperatures, as in large alternators and motors.

As can be seen in the Carbon-Cu phase diagram, the solubility of carbon in copper is extremely low (approximately $0.0001 \mathrm{wt} \% \mathrm{C}$ at $1100{ }^{\circ} \mathrm{C}$, which is above its melting point). Furthermore, it was observed during milling and compaction of $\mathrm{Cu}$ and MWCNTs that there was very little cohesion between the components. Additionally, it was felt that poor wetability and lack of interfacial bonding could be improved by prolonging the duration of compaction and by extending the time at the holding temperature during firing with the addition of $\mathrm{Ni}$ and $\mathrm{Ag}$..

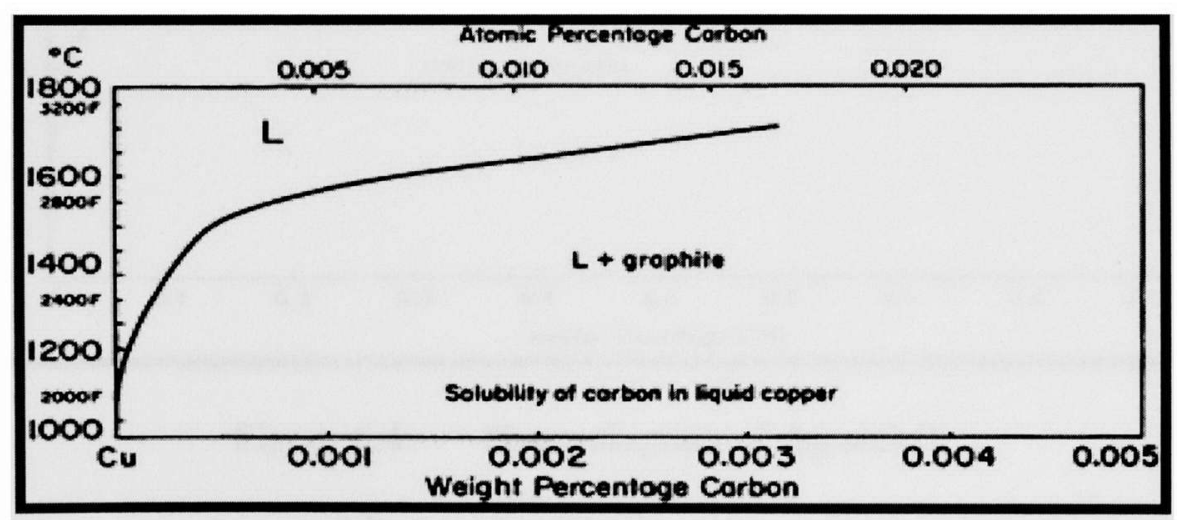

Figure 6.3: (a) Cu-C phase diagram showing the solubility of carbon in copper 


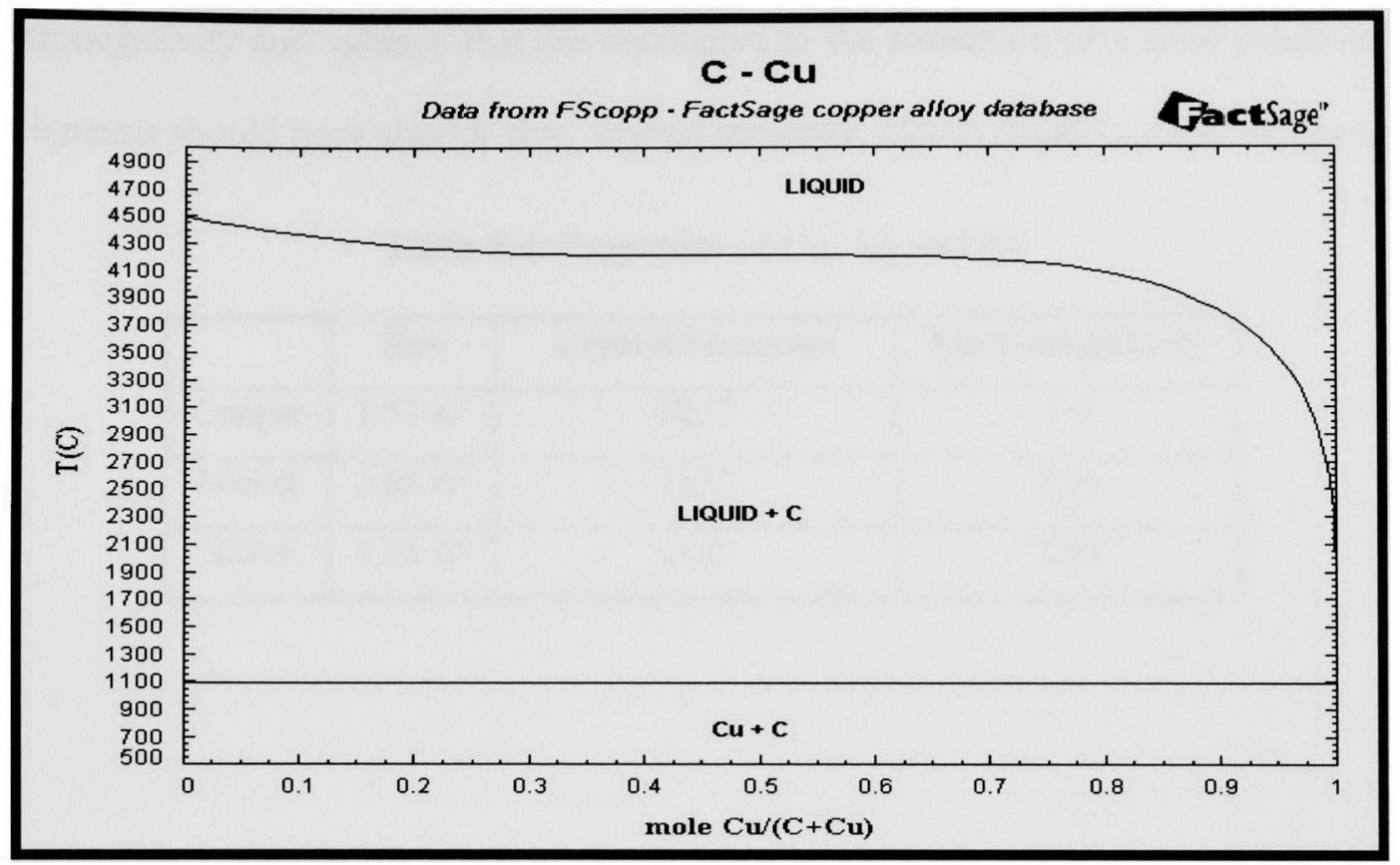

Figure 6.3:(b) Cu-C phase diagram showing the solubility of carbon in copper

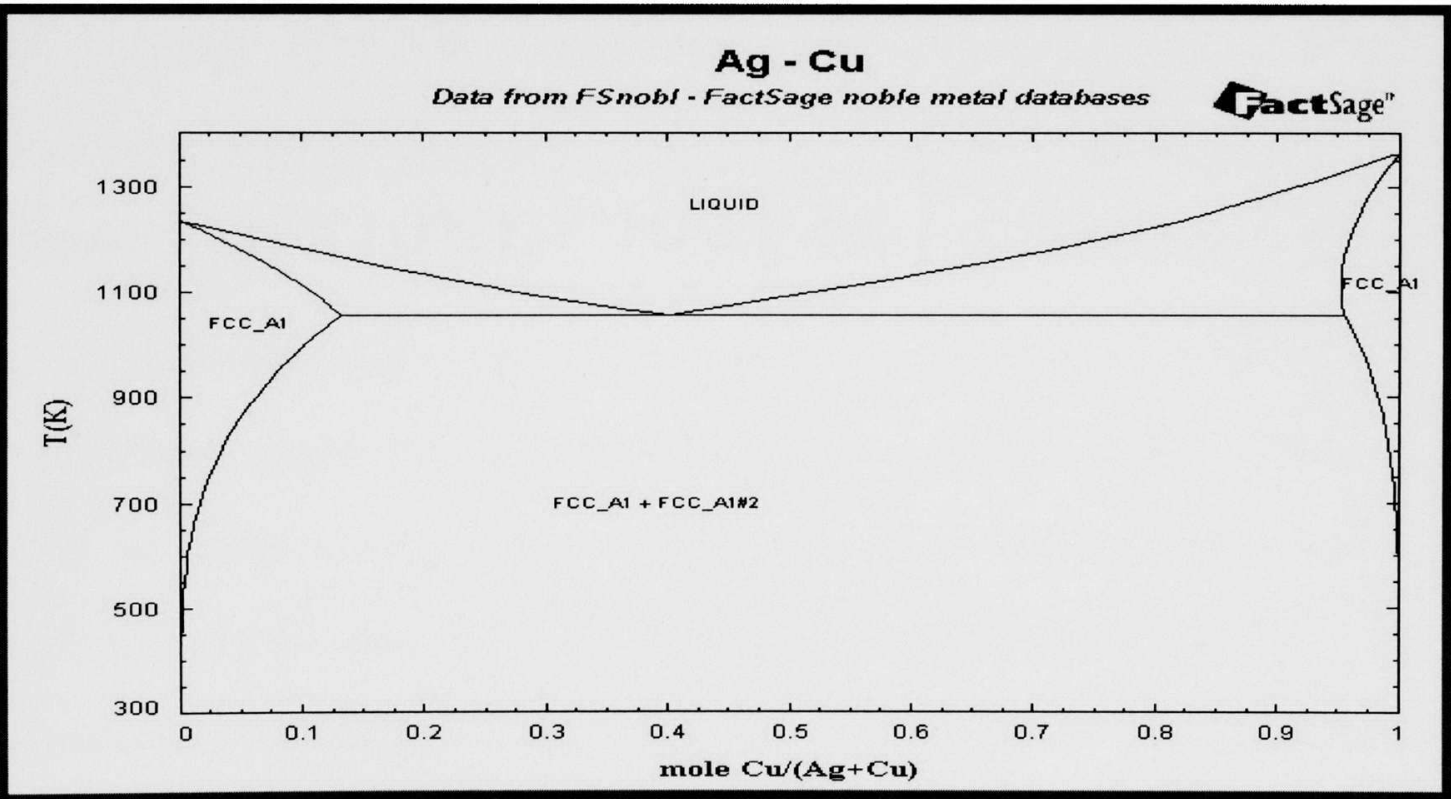

Figure 6.4: Phase diagram of $\mathrm{Ag}$ and $\mathrm{Cu}$

Furthermore, it was felt that the alloying elements added to the copper matrix should form a solid solution. Table 6.3, shows the parameters of size, crystal structure, 
electronegativity and valency that are conducive to the formation of a solid solution. All all elements should have similar size, crystal structure, electronegativity and valency.

Table 6.4: Properties of $\mathrm{Cu}, \mathrm{Ag}$ and $\mathrm{Ni}$

\begin{tabular}{|c|c|c|c|}
\hline & Size & Crystal Structure & Electronegativity \\
\hline Copper & $1.57 \mathrm{~A}^{\circ}$ & FCC & 1.9 \\
\hline Nickel & $1.62 \mathrm{~A}^{\circ}$ & FCC & 1.91 \\
\hline Silver & $1.75 \mathrm{~A}^{\circ}$ & FCC & 1.93 \\
\hline
\end{tabular}




\section{EXPERIMENTAL PROCEDURE}

\subsection{Sample Preparation}

\subsection{1. $\quad$ Milling}

High energy ball milling (HEBM) was conducted using a SPEX 8000 mill with a hardened steel vial ( $(\varphi 21 / 4$ in $\times 3$ in) as the milling container and stainless steel balls as grinding medium. Pure copper powder (99.99\%) of 1-5 micron size was poured into the milling container while in the glove box, in an effort to trap argon in the container. A 10:1 BPR or charge ratio was maintained with grinding medium consisting of steel balls ( $\varphi$ 1/2 in) for effective milling. Milling was conducted for a milling time of 100 minutes.

\subsection{2. $\quad$ Mixing}

$4 \mathrm{~g}$ mixtures of MWCNT-CuAgNi at volume percentages of $8 \%, 12 \%$ and $16 \%$ of MWCNTS were prepared by placing copper powder that was previously milled for about 100 minutes, and mixing silver resinate-(25\%), nickel resinate-(8\%) and MWCNT's (diameter $10-20 \mathrm{~nm}$ and length $0.5-40 \mu \mathrm{m}$ ) into the milling container under argon. .

All adding was conducted in the glove box. The chambers of the glove box were evacuated and purged with argon gas in an effort to prevent oxidation of the powders which are highly reactive at such small particle sizes. Mixing of this powder was conducted using the same SPEX 8000 mill with a hardened steel vial $(\varphi 21 / 4$ in $\times 3$ in) and stainless steel balls ( $\varphi$ 1/4 in) at a BPR of 5:1 for effective mixing. The duration for mixing was 5 minutes. The powdered composite was then removed from the vial and stored in an air tight bottle within the glovebox under argon. 
Table 7.1 shows the actual weights of each element that were used for each volume fraction of MWCNT in the composite.

Table 7.1: Weight of individual elements in the composite pellet sample

\begin{tabular}{|c|c|c|c|}
\hline Cu (grams) & Ag (grams) & Ni (grams) & $\begin{array}{c}\text { MWCNT } \\
\text { (grams) }\end{array}$ \\
\hline $3.63(91 \mathrm{wt} \%)$ & $0.14(3 \mathrm{wt} \%)$ & $0.03(1 \mathrm{wt} \%)$ & $0.2(5 \mathrm{wt} \%)$ \\
\hline $3.69(92 \mathrm{wt} \%)$ & $0.15(4 \mathrm{wt} \%)$ & $0.027(1 \mathrm{wt} \%)$ & $0.133(3 \mathrm{wt} \%)$ \\
\hline $3.73(93 \mathrm{wt} \%)$ & $0.15(4 \mathrm{wt} \%)$ & $0.03(1 \mathrm{wt} \%)$ & $0.09(2 \mathrm{wt} \%)$ \\
\hline
\end{tabular}

\subsubsection{Pressing/Consolidation}

A stainless steel die was specially designed and manufactured for the consolidation of the milled powders into pellets. The milled powder was carefully pored into the bore of the die while in the glovebox. Finally the powder was uni-axially cold pressed at $669.4 \mathrm{MPa}(20,000 \mathrm{lbs}$ force $)$ using a manual pellet press and held at that pressure for about 30 minutes to form the pellet. The pellet was then carefully ejected from the die and stored in an airtight sample bag.

\subsection{4. $\quad$ Sintering}

Sintering of the pellet was conducted in a Lindberg $\left(1100{ }^{\circ} \mathrm{C}\right)$ High Temperature Tube Furnace at $950{ }^{\circ} \mathrm{C}$. This temperature was well below the melting point temperature of copper $\left(1030^{\circ} \mathrm{C}\right)$. The samples were sintered under argon in order to avoid oxidation, utilizing a ramp time of 30 minutes and a hold time of 2.5 hours. The sintered pellet was then allowed to cool down gradually to room temperature. 


\subsection{Analytical Techniques}

\subsubsection{Metallograpy}

A JEOL Scanning Electron Microscope (SEM) equipped with Energy Dispersive Spectrometer (EDS) was used to determine the particle size and the MWCNT distribution in the pellet. SEM analysis was employed to analyze the phase distribution and composition of the pertinent phases of the milled powders and sintered pellets. All of the SEM analyses were conducted using secondary electrons.

\subsubsection{Raman Spectroscopy}

Raman spectroscopy analyses were conducted using monochromatic light.to analyzes the structure of the carbon nanotubes before and after sintering. Raman spectra were recorded using a high-resolution (maximum $\sim 50 \mathrm{~cm}^{-1}$ ) $\mathrm{f} / 1.8$ double monochromator. The PM dark current was quoted at $13 \mathrm{MWatts}$ and laser excitation to be $514 \mathrm{~nm}$. The so-called G-line is a characteristic feature of the graphitic layers and corresponds to the tangential vibration of the carbon atoms. The second characteristic mode (D-line) is a typical indicator of defective graphitic structures. A comparison of the ratios of these two peak intensities provides a measure of the quality of the sample. The purpose of using Raman Spectroscopy was to determine whether any significant destruction of the MWCNTs occurred during mixing and sintering of the composite.

\subsubsection{Electrical Conductivity}

The four probe electrical resistivity meter (described in Chapter 4) was used to determine the electrical conductivity of the pellets. The electrical resistivity of the pellet was first calculated by using the following formula: 
$\rho=(\Pi \mathrm{t} / \ln 2) \mathrm{V} / \mathrm{I} \mu \mathrm{Ohm}-$ Centimeters for $\mathrm{s}>>\mathrm{t}$

where $\rho$ is the resistivity, $\mathrm{V}$ is the voltage, I is the current, $\mathrm{s}$ is the spacing between the probes, and $\mathrm{t}$ is the thickness.

The electrical conductivity was then calculated as the reciprocal of electrical resistivity. In order to determine the effect of sintering on porosity and electrical conductivity of the composite, measurements were conducted before and after sintering.

\subsubsection{Porosity}

Porosity $(\mathrm{P})$ of the pellets was determined by adopting the procedure described in Chapter 3, where $\mathrm{P}$ is calculated using the following formula:

$$
P=\frac{W_{2}-W_{1}}{\left(W_{2}-W_{3}\right) \rho}
$$

As previously mentioned in section 7.2.3., the porosity of the composite was determined before and after sintering to determine its effect on electrical conductivity.

\subsection{Experimental Results}

7.3.1. Electrical Resistance Graphs of Un-Sintered Pellets

Figure 7.1 shows a typical plot of the resistance of the composite versus temperature as obtained from the four probe resistivity meter. The actual resistance values were obtained from LABVIEW software and the imported into Microsoft Excel to produce the graph.

The below graph shows a slight increase in the resistance with increase in temperature, for various compositions of the un-sintered composite. This is an indication 
of the metallic nature of the composite. A regression analysis was conducted to test the best fit of resistance measurements obtained.

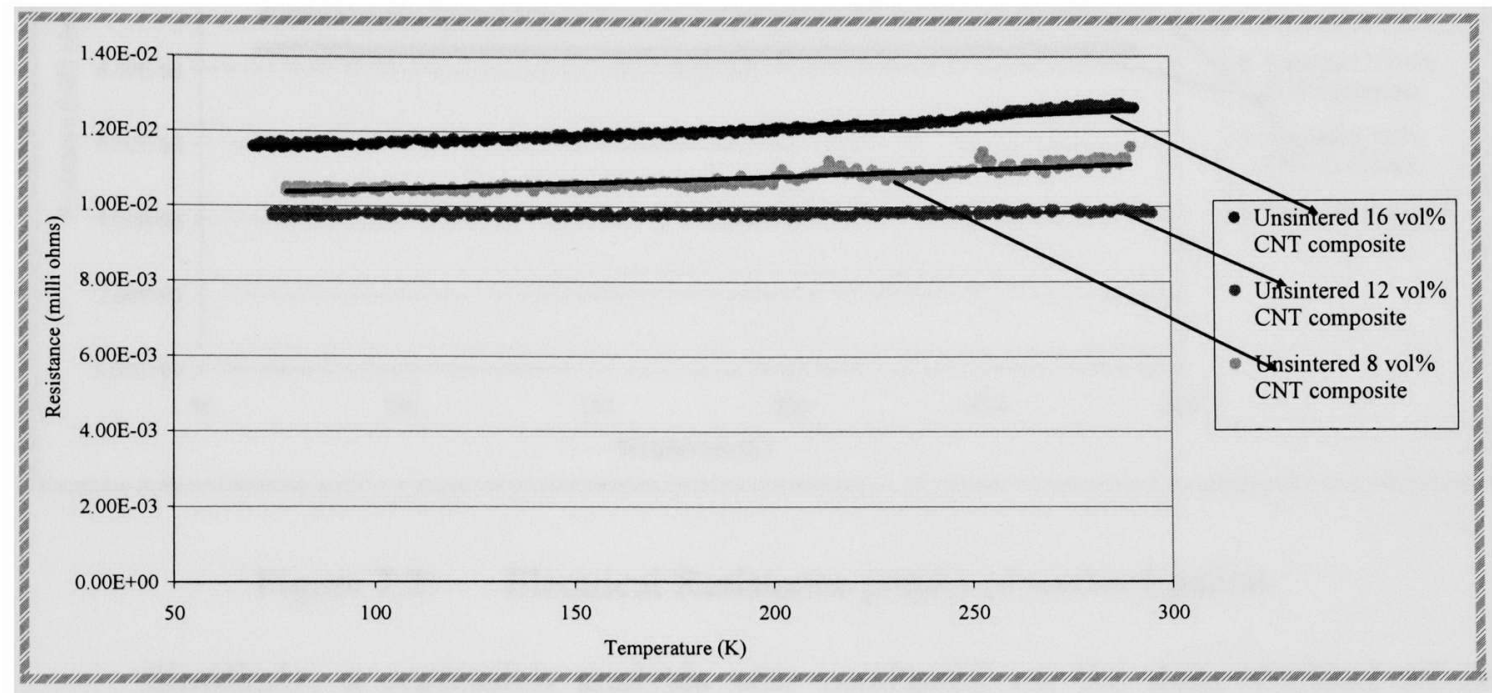

Figure 7.1: Electrical Resistance of un-sintered pellets

The equation for best fit line of the resistance for each composite was determined as follows:

$16 \mathrm{Vol} \% \mathrm{MWCNT}$ : equation for best fit line $=4 \mathrm{E}^{-06} \mathrm{x}+0.0112$

$12 \mathrm{Vol} \%$ MWCNT: equation for best fit line $=3 \mathrm{E}^{-06} \mathrm{x}+0.0101$

8 Vol \% MWCNT: equation for best fit line $=4 \mathrm{E}^{-06} \mathrm{x}+0.0117$

The above equations have Root Mean Square (RMS) of $0.9499,0.8086$ and 0.9846 respectively, which are close to unity. This indicated that the resistance data has a good fit with the equation for the best fitted line.

7.3.2. Electrical Resistance Graphs of sintered Pellets 


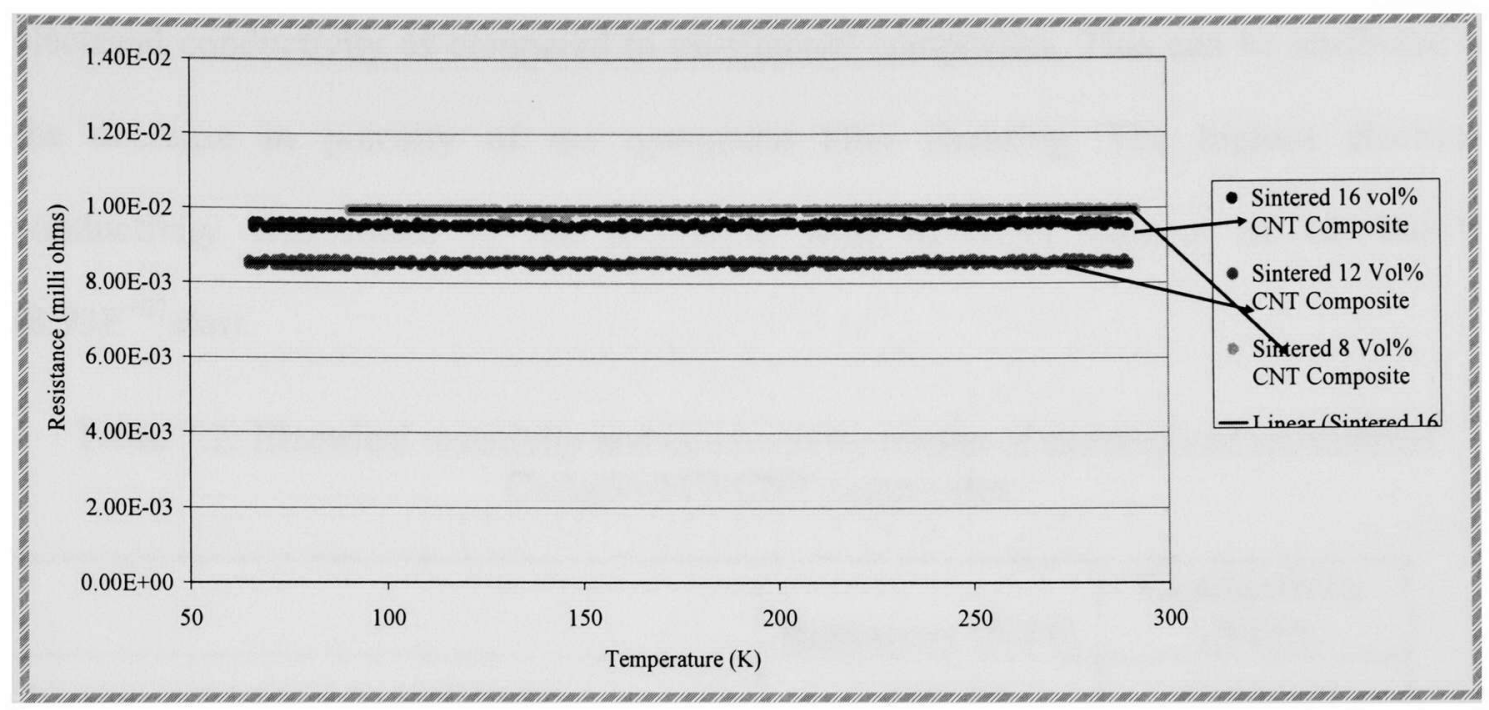

Figure 7.2: Electrical Resistance graphs of sintered pellets

Simillarly, a regression analysis was conducted on the data obtained of the resistance of sintered composites and the equations for the best fit line obtained were as follows:

$16 \mathrm{Vol} \% \mathrm{MWCNT}$ : equation for best fit line $=4 \mathrm{E}^{-06} \mathrm{x}+0.01186$

12 Vol \% MWCNT: equation for best fit line $=4 \mathrm{E}^{-06} \mathrm{x}+0.0119$

$8 \mathrm{Vol} \%$ MWCNT: equation for best fit line $=4 \mathrm{E}^{-06} \mathrm{x}+0.01175$

The RMS values of the above equations were $0.9899,0.99808$ and 0.9846 respectively, which again were close to unity. This indicated that the resistance data has a good fit with the equation for the best fitted line.

The room temperature electrical resistivity of all the sintered and un-sintered CuAgNi-MWCNT composites was calculated using the formula described in Section 7.2.3. The electrical conductivity was then calculated as the reciprocal of electrical resistivity. It can be observed in Table 7.2 that the sintered composites possessed higher 
electrical conductivity as compared to un-sintered composites. This can be attributed to the decrease in porosity of the composite after sintering. The highest electrical conductivity was found in the composite with MWCNT content of $12 \mathrm{Vol} \%$ $\left(8.93 \mathrm{E}^{+07} \mathrm{~s} / \mathrm{m}\right)$.

Table 7.2: Electrical resistivity and conductivity results of sintered and un-sintered $\mathrm{CuAgNi}-\mathrm{MWCNT}$ composites

\begin{tabular}{|l|c|c|}
\hline & Resistivity (NTP) & $\begin{array}{c}\text { Conductivity } \\
\text { (NTP) }\end{array}$ \\
\hline Unsintered 16 Vol \% MWCNT & $1.66 \mathrm{E}-08$ & $6.02 \mathrm{E}+07$ \\
\hline Unsintered 12 Vol \% MWCNT & $1.29 \mathrm{E}-08$ & $7.75 \mathrm{E}+07$ \\
\hline Unsintered 8 Vol \% MWCNT & $1.52 \mathrm{E}-08$ & $6.58 \mathrm{E}+07$ \\
\hline Sintered 16 Vol \% MWCNT & $1.25 \mathrm{E}-08$ & $7.98 \mathrm{E}+07$ \\
\hline Sintered 12 Vol \% MWCNT & $1.12 \mathrm{E}-08$ & $8.93 \mathrm{E}+07$ \\
\hline Sintered 8 Vol \% MWCNT & $1.31 \mathrm{E}-08$ & $7.63 \mathrm{E}+07$ \\
\hline Pellet from as received copper powder & $1.71 \mathrm{E}-08$ & $5.85 \mathrm{E}+07$ \\
\hline Pellet from milled copper powder & $1.69 \mathrm{E}-08$ & $5.91 \mathrm{E}+07$ \\
\hline Copper plate & $1.68 \mathrm{E}-08$ & $5.94 \mathrm{E}+07$ \\
\hline Copper (std ref*) & $1.68 \mathrm{E}-08$ & $5.96 \mathrm{E}+07$ \\
\hline
\end{tabular}

Table 7.3 shows the porosities of both un-sintered and sintered composites. For both sintered and unsintered composites, the lowest porosity was observed in the composite with $12 \mathrm{Vol} \% \mathrm{MWCNT}$. This may be attributed to the optimal packing of MWCNT particles into the interstitial sites located between $\mathrm{Cu}$ particles. As mentioned in Chapter 4, the electrical conductivity of materials generally decreases with an increase in porosity. 
Table 7.3: Porosity results of sintered and un-sintered pellets

\begin{tabular}{|c|c|c|}
\hline $\begin{array}{c}\text { Volume \% } \\
\text { MWCNT }\end{array}$ & $\begin{array}{c}\text { Porosity un-sintered } \\
\text { composites }\end{array}$ & $\begin{array}{c}\text { Porosity sintered } \\
\text { composites }\end{array}$ \\
\hline 16 & 0.2572 & 0.1951 \\
\hline 12 & 0.1661 & 0.1274 \\
\hline 8 & 0.1830 & 0.1786 \\
\hline
\end{tabular}

The electrical conductivities of composites before and after sintering are shown in Figure 7.3 where it was observed that the highest conductivity occurred in the composite (12 Vol \% MWCNT) with the lowest porosity $(0.1274)$. It should also be noted that the porosities of the sintered composites were lower than those of the unsintered composites. This may be attributed to the filling of microvoids in the sintered composite by molten material.

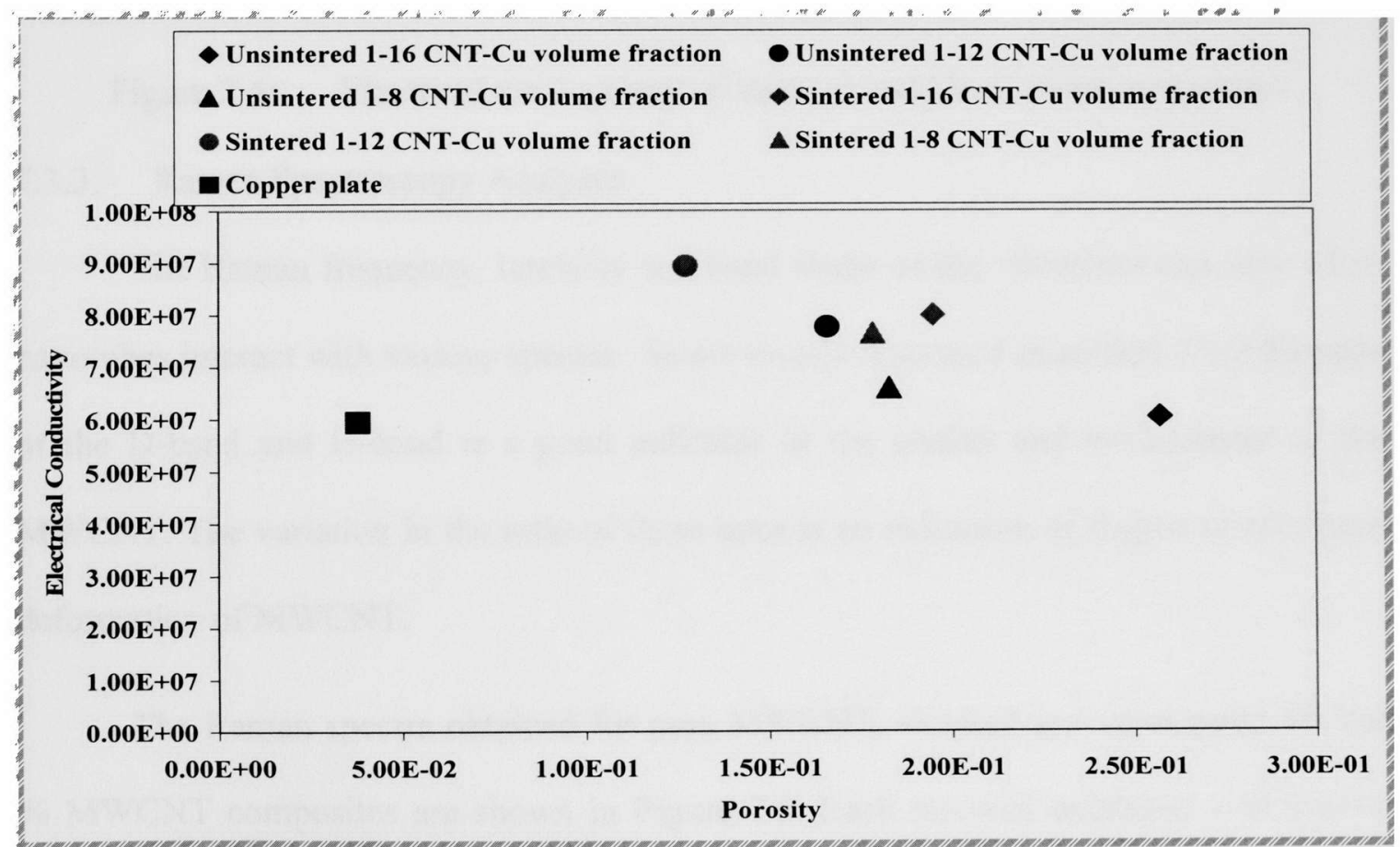

Figure 7.3: Electrical conductivity of sintered and Un-sintered composites 
From the bar diagram (Figure 7.4), it can be observed that the electrical conductivity of the $16 \mathrm{Vol} \% \mathrm{MWCNT}$ sintered composite was $50 \%$ greater than that of pure copper. The $12 \mathrm{Vol} \%$ and $8 \mathrm{Vol} \%$ MWCNT sintered composites possessed electrical conductivities that were $34 \%$ and $28 \%$ greater than that of pure $\mathrm{Cu}$.

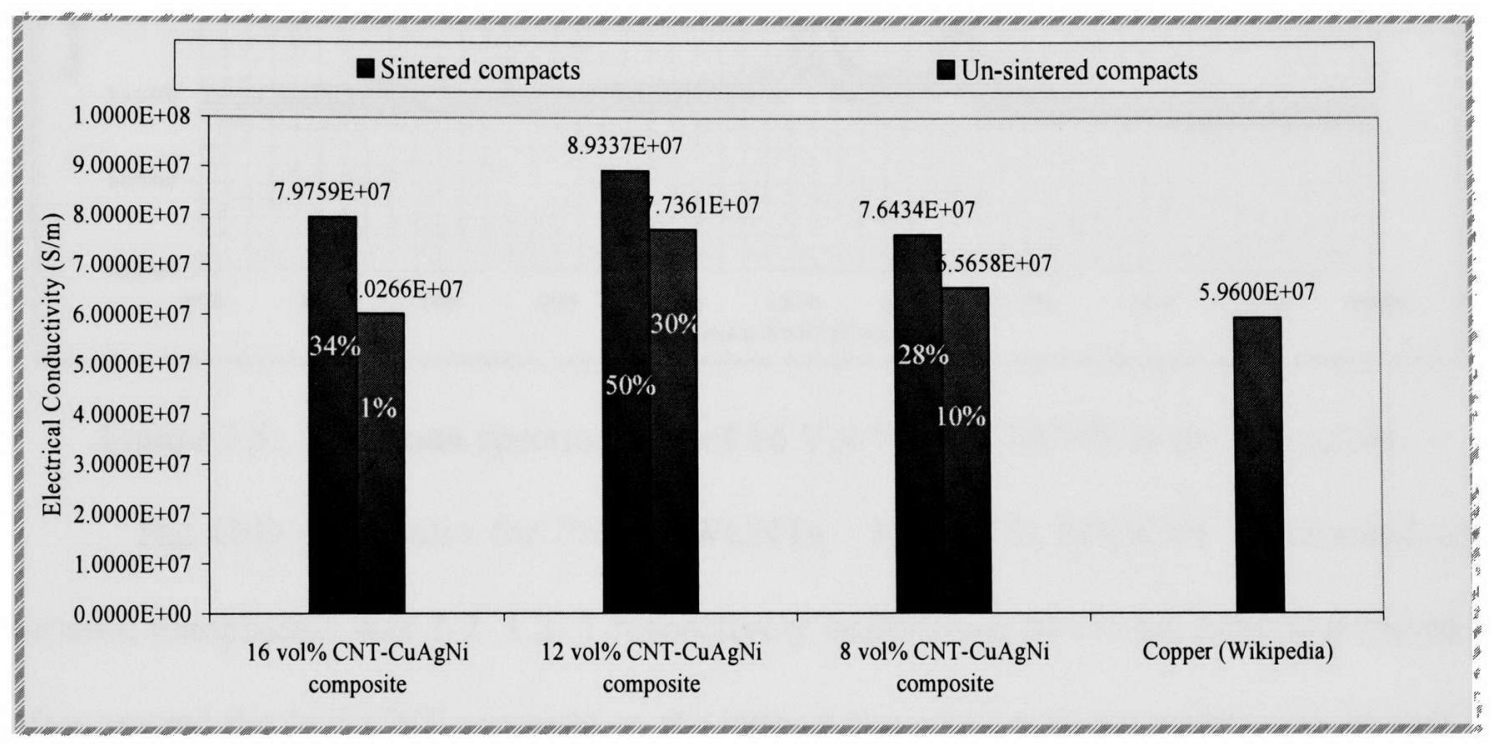

Figure 7.4: Electrical conductivity of sintered and Un-sintered composites

\subsubsection{Raman Spectroscopy Analyses}

The Raman frequency, intensity and band shape of the vibrations can vary when nanotubes interact with various species. As previously discussed in section 7.2.2 the ratio of the D-band and G-band is a good indicator of the quality and environment of the MWCNT. The variation in the ratio of these lines is an indication of degree of structural deformation of MWCNT.

The Raman spectra obtained for pure MWCNT, sintered and un-sintered $16 \mathrm{Vol}$ $\%$ MWCNT composites are shown in Figure 7.5. Each material exhibited well known disordered carbon band (D peak) and highly ordered graphite band ( $\mathrm{G}$ peak) 


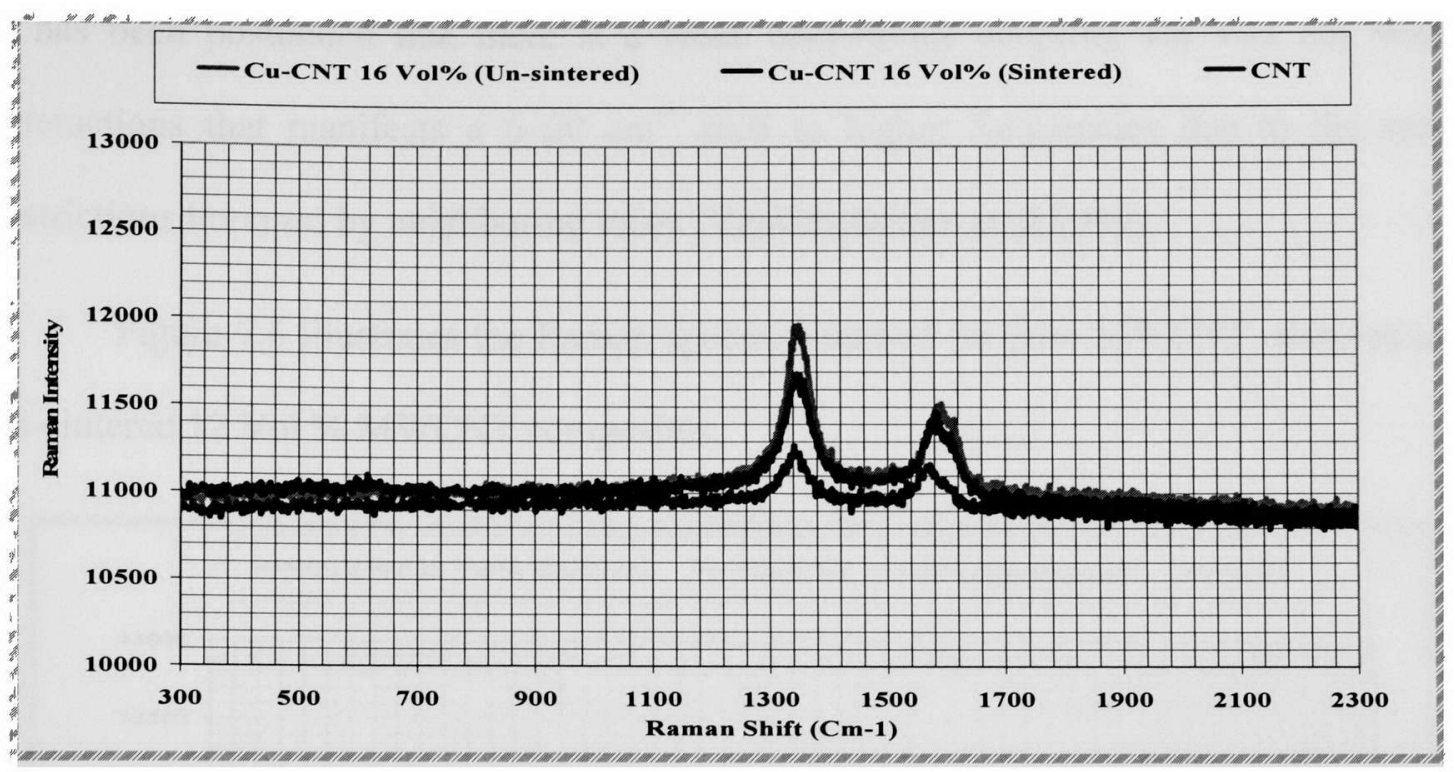

Figure 7.5: Raman spectroscopy of $16 \mathrm{Vol} \%$ of MWCNT in the composite

The G:D peak ratio for Pure MWCNTs, 16 Vol \% MWCNT un-sintered and sintered composites was $1.7,1.2,1$ respectively confirming that some structural changes of/or around the MWCNT occurred as the latter was mixed with the composite as well as after sintering. In figure 7.5, the pure MWCNT, $16 \mathrm{Vol} \% \mathrm{MWCNT}$ un-sintered and sintered composites exhibited G-peaks at $1575 \mathrm{~cm}^{-1}, 1585 \mathrm{~cm}^{-1}$ and $1592 \mathrm{~cm}^{-1}$ respectively. The values of both $\mathrm{G}$ and $\mathrm{D}$ peaks are tabulated in Table 7.4.

Table 7.4: Raman spectroscopy of $16 \mathrm{Vol} \%$ of MWCNT in the composite

\begin{tabular}{|l|l|l|}
\hline & D Peak & G Peak \\
\hline Pure MWCNT & $1351 \mathrm{~cm}^{-1}$ & $1575 \mathrm{~cm}^{-1}$ \\
\hline 16 Vol \% MWCNT (Un-sintered) & $1352 \mathrm{~cm}^{-1}$ & $1585 \mathrm{~cm}^{-1}$ \\
\hline 16 Vol \% MWCNT (Sintered) & $1352 \mathrm{~cm}^{-1}$ & $1592 \mathrm{~cm}^{-1}$ \\
\hline
\end{tabular}

The G peak shift of 10 and $17 \mathrm{~cm}^{-1}$ that were obtained for $16 \mathrm{Vol} \%$ MWCNT unsintered and sintered composites respectively with respect to that of pure MWCNTs, is an indication of a change in the structural environment of MWCNT. In nanotube aggregates, 
it has been postulated that there is a weak inter-tubule coupling via Van der Waals interactions that manifests a $6-20 \mathrm{~cm}^{-1}$ shift to higher frequencies due to the space restrictions imposed by neighboring tubes (Venkateswaran et al.1999).

Figure 7.6 illustrates the Raman spectra obtained for pure MWCNT, sintered and un-sintered $12 \mathrm{Vol} \%$ MWCNT composites.

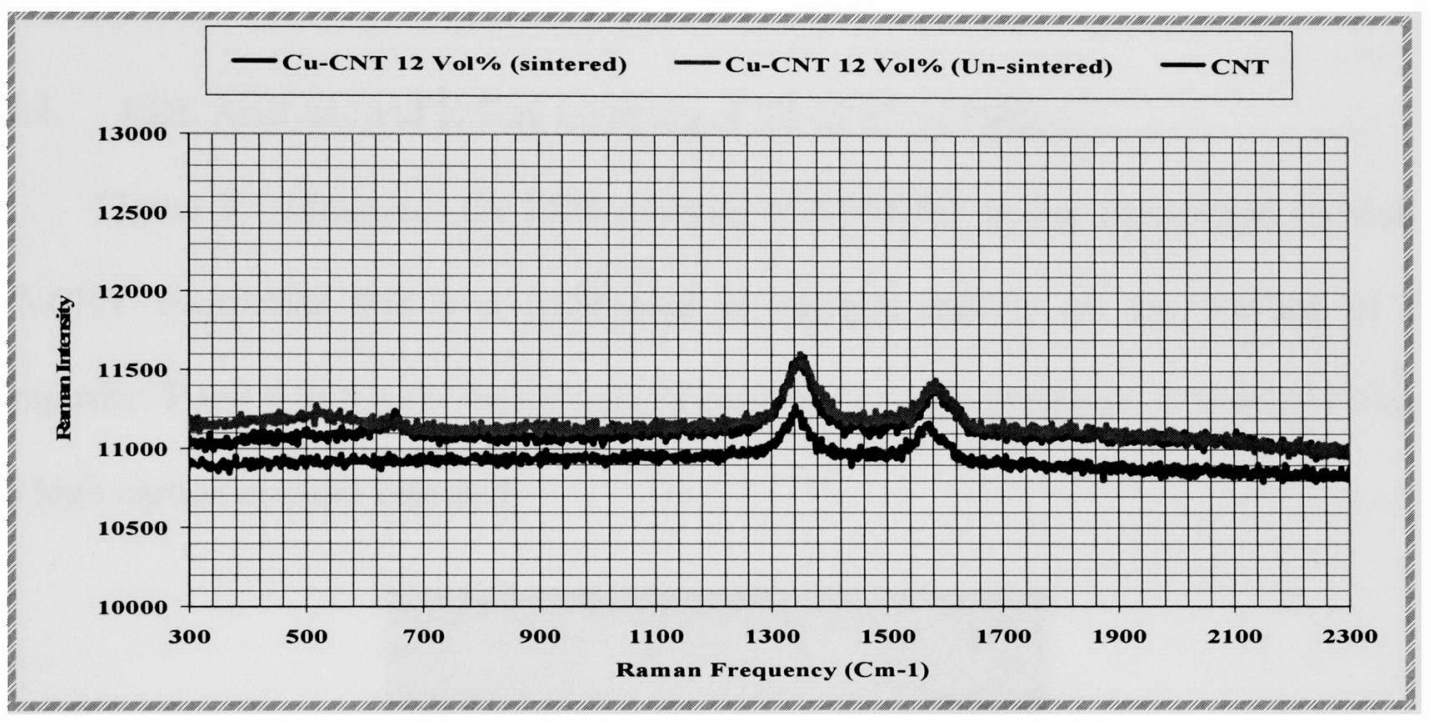

Figure 7.6: Raman spectroscopy of $12 \mathrm{Vol} \%$ of MWCNT in the composite In this case, the G:D peak ratios for Pure MWCNTs, 12 Vol \% MWCNT unsintered and sintered composites were $1.7,1.7$ and 1.7 respectively and the G-peaks occurred at $1575 \mathrm{~cm}^{-1}, 1584 \mathrm{~cm}^{-1}$, and $1584 \mathrm{~cm}^{-1}$ respectively. The values of both $\mathrm{G}$ and D peaks are summarized in Table 7.5

Table 7.5: $\quad$ D and G peaks of MWCNT and $12 \mathrm{Vol} \%$ composites

\begin{tabular}{|l|l|l|}
\hline & D Peak & G Peak \\
\hline Pure MWCNT & $1351 \mathrm{~cm}^{-1}$ & $1575 \mathrm{~cm}^{-1}$ \\
\hline Cu-MWCNT 16 Vol \% (Un-sintered) & $1356 \mathrm{~cm}^{-1}$ & $1584 \mathrm{~cm}^{-1}$ \\
\hline Cu-MWCNT 16 Vol \% (Sintered) & $1356 \mathrm{~cm}^{-1}$ & $1584 \mathrm{~cm}^{-1}$ \\
\hline
\end{tabular}


The G peak shift for both sintered and un-sintered $12 \mathrm{Vol} \%$ MWCNT was $9 \mathrm{~cm}$ 1 relative to that of pure MWCNTs. This indicated that there was very little structural rearrangement with regard to the MWCNT in the composite of $12 \mathrm{Vol} \%$ MWCNT compositions. This is in contrast to shifts of 10 and $17 \mathrm{~cm}-1$ that were obtained for sintered and un-sintered $16 \mathrm{Vol} \% \mathrm{MWCNT}$ respectively which indicated some structural rearrangement of MWCNT upon sintering.

\subsubsection{EDS Analysis and X-Ray mapping of Un-sintered Samples}

Figure 7.7 illustrates the EDS analysis of MWCNT in the un-sintered $16 \mathrm{Vol} \%$ MWCNT composite that was performed at various regions on the surface of the composite. Point 4 in the photomicrograph demonstrates the presence of MWCNT due to the high carbon content detected.

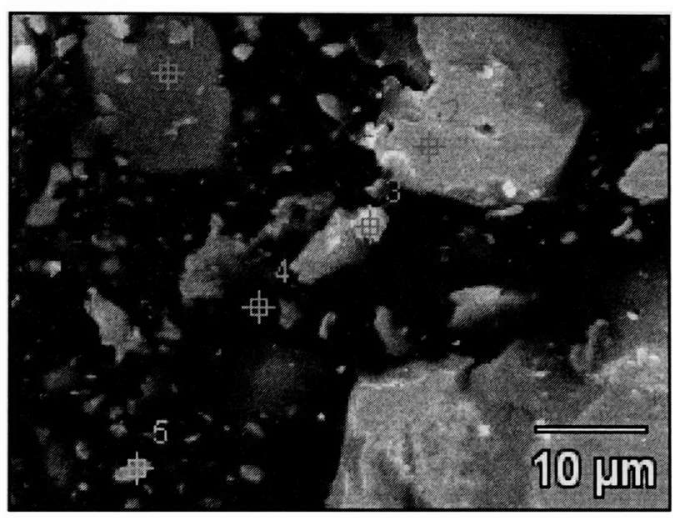

\begin{tabular}{|c|c|c|c|c|c|c|c|c|c|c|c|c|}
\hline \multicolumn{3}{|c|}{ C-K } & & \multicolumn{1}{c|}{ Ni-K } & & & Cu-K & & & & Ag-L \\
\hline & W \% & A \% & C \% & W \% & A \% & $\%$ & W \% & A \% & C \% & W \% & $\begin{array}{c}\text { \% } \\
\text { \% }\end{array}$ & C \% \\
\hline pt1 & 77.7 & 95.0 & 77.7 & 0.5 & 0.1 & 0.5 & 20.0 & 4.6 & 20.0 & 20.0 & 0.2 & 1.8 \\
\hline pt2 & 15.1 & 46.6 & 15.1 & 1.2 & 0.7 & 1.2 & 81.2 & 47.5 & 81.2 & 81.2 & 0.1 & 0.4 \\
\hline pt3 & 54.5 & 85.5 & 54.5 & 0.6 & 0.2 & 0.6 & 41.4 & 12.3 & 41.4 & 41.4 & 0.3 & 1.9 \\
\hline pt4 & 92.8 & 98.7 & 92.8 & 1.9 & 0.4 & 1.9 & 3.8 & 0.8 & 3.8 & 3.8 & 0.2 & 1.5 \\
\hline pt5 & 62.3 & 89.4 & 62.3 & 0.3 & 0.1 & 0.3 & 36.4 & 9.9 & 36.4 & 0.4 & 0.1 & 0.4 \\
\hline
\end{tabular}

Fig 7.7: $\quad$ EDS analysis of un-sintered 16 Vol \% MWCNT composite 
The X-ray mapping shown in Figure 7.8 depicts the presence of carbon (black region), copper (grey region). As shown in the other X-Ray maps, nickel and silver were evenly distributed within the composite.
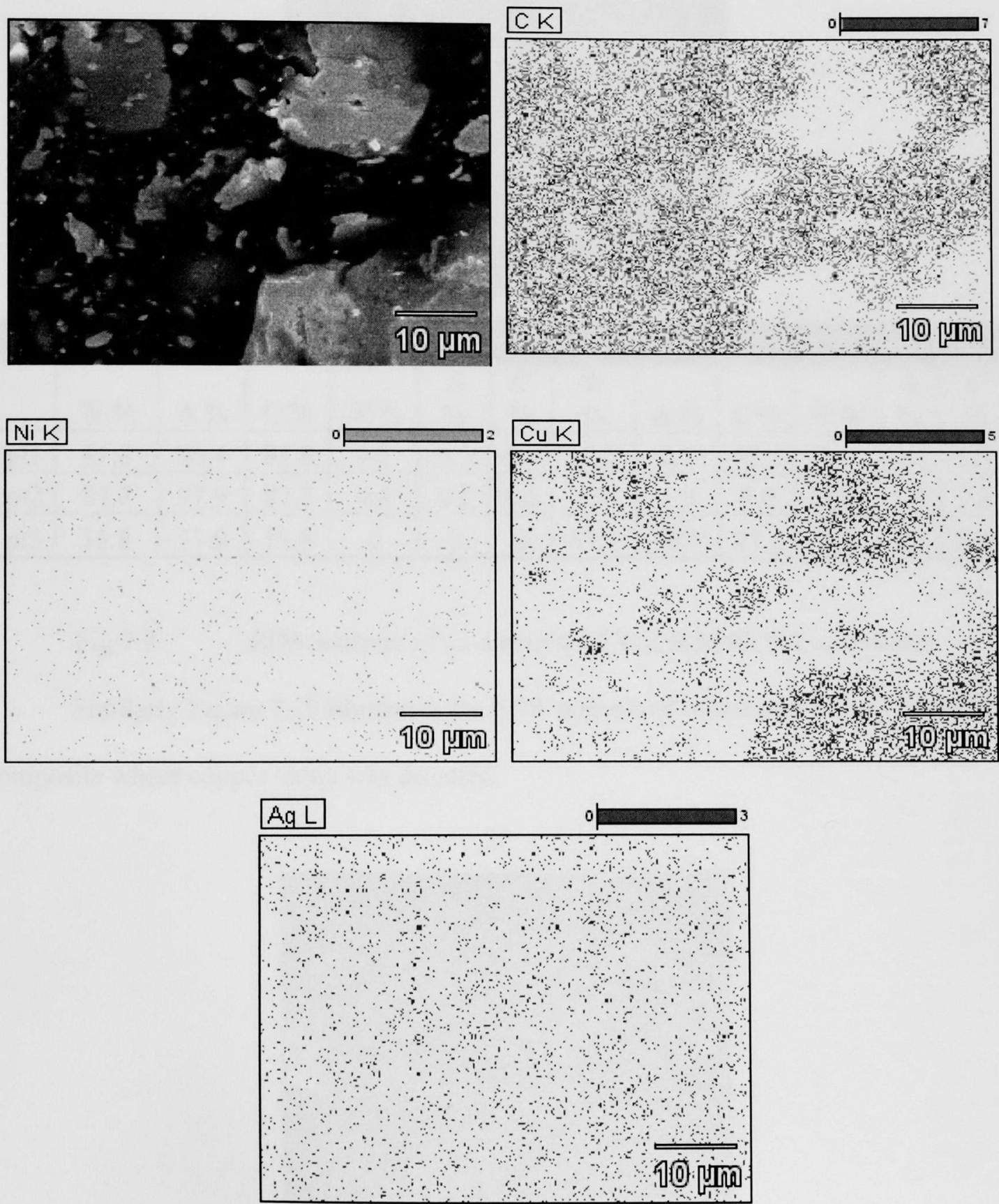

Figure 7.8: $\quad$ X-Ray mapping of un-sintered $16 \mathrm{Vol} \%$ MWCNT composite 
Figure 7.9 illustrates the EDS analysis of MWCNT in the un-sintered $12 \mathrm{Vol} \%$ MWCNT composite that was performed at various locations on the surface of the composite. Note the presence of MWCNT at points 1 and 2.

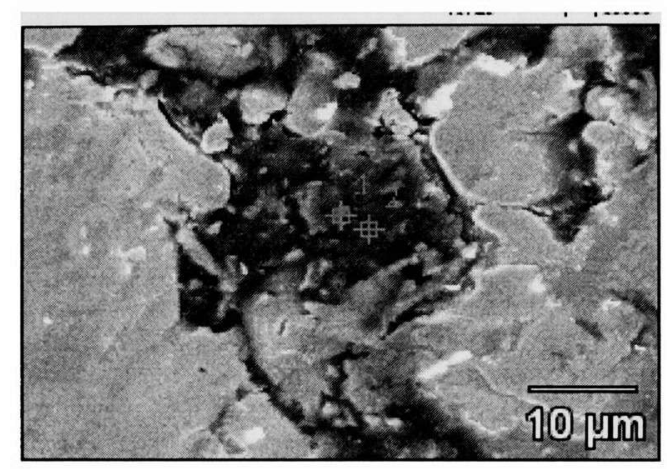

\begin{tabular}{|c|c|c|c|c|c|c|c|c|c|c|c|c|}
\hline \multicolumn{3}{|c}{$\boldsymbol{C}-\boldsymbol{K}$} & \multicolumn{4}{c}{$\boldsymbol{N i - K}$} & \multicolumn{4}{c|}{$\boldsymbol{C}-\boldsymbol{K}$} & \multicolumn{3}{c|}{$\boldsymbol{A g}-\boldsymbol{L}$} \\
\hline & W \% & A \% & C \% & W \% & $\begin{array}{c}\text { A } \\
\%\end{array}$ & $\begin{array}{c}\text { C } \\
\%\end{array}$ & $\begin{array}{c}\text { W } \\
\%\end{array}$ & A \% & C \% & W \% & $\begin{array}{c}\text { A } \\
\%\end{array}$ & $\begin{array}{c}\text { C } \\
\%\end{array}$ \\
\hline pt1 & 81.8 & 96.4 & 81.8 & 0.7 & 0.2 & 0.7 & 12.2 & 2.7 & 12.2 & 5.3 & 0.7 & 5.3 \\
\hline pt2 & 87.7 & 97.9 & 87.7 & 0.6 & 0.2 & 0.6 & 5.9 & 1.3 & 5.9 & 5.8 & 0.7 & 5.8 \\
\hline pt3 & 11.8 & 37.9 & 11.8 & 0 & 0 & 0 & 83.1 & 50.5 & 83.1 & 0.5 & 0.2 & 0.5 \\
\hline
\end{tabular}

Fig 7.9: $\quad$ EDS analysis of un-sintered 12 Vol \% MWCNT composite

Similarly Figure 7.10 illustrates the EDS analysis of unsintered 8 Vol \% MWCNT composite where copper alone was detected.

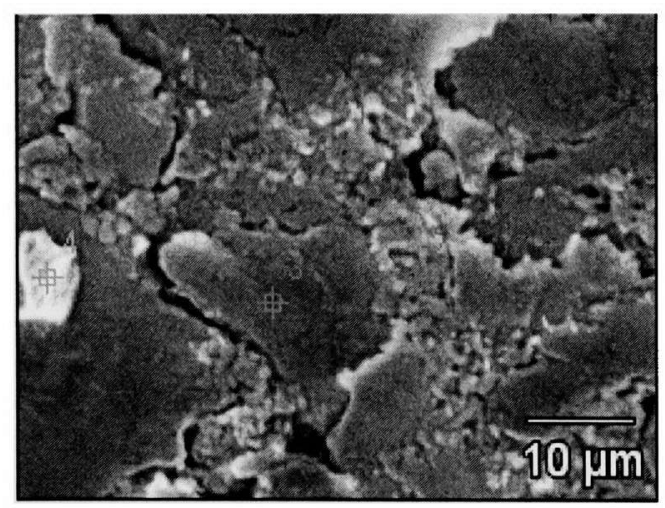




\begin{tabular}{|c|c|c|c|c|c|c|c|c|c|c|c|c|}
\hline & \multicolumn{3}{|c|}{$C-K$} & \multicolumn{3}{|c|}{$N i-K$} & \multicolumn{3}{|c|}{$\mathrm{Cu}-\mathrm{K}$} & \multicolumn{3}{|c|}{$A g-L$} \\
\hline & $W \%$ & $A \%$ & $C \%$ & $W \%$ & $A \%$ & C\% & W\% & $A \%$ & $C \%$ & $W \%$ & $A \%$ & $C \%$ \\
\hline$p t I$ & 26.5 & 62.66 & 26.5 & 0.66 & 0.32 & 0.66 & 60.06 & 26.84 & 60.06 & 8.27 & 2.18 & 8.27 \\
\hline$p t 2$ & 35.4 & 73.58 & 35.4 & 0.38 & 0.16 & 0.38 & 52.05 & 20.45 & 52.05 & 9.92 & 2.3 & 9.92 \\
\hline$p t 3$ & 21.08 & 56.49 & 21.08 & 0 & 0 & 0 & 75.08 & 38.03 & 75.08 & 1.32 & 0.39 & 1.32 \\
\hline$p t 4$ & 27.75 & 58.39 & 27.75 & 11.64 & 5.01 & 11.64 & 20.98 & 8.34 & 20.98 & 0.77 & 0.18 & 0.77 \\
\hline
\end{tabular}

Fig 7.10: $\quad$ EDS analysis of un-sintered 8 Vol \% MWCNT composite

The X-ray mappings in Figure 7.11 indicate a homogeneous distribution of MWCNT, $\mathrm{Cu}, \mathrm{Ni}$ and $\mathrm{Ag}$ within the un-sintered composite.

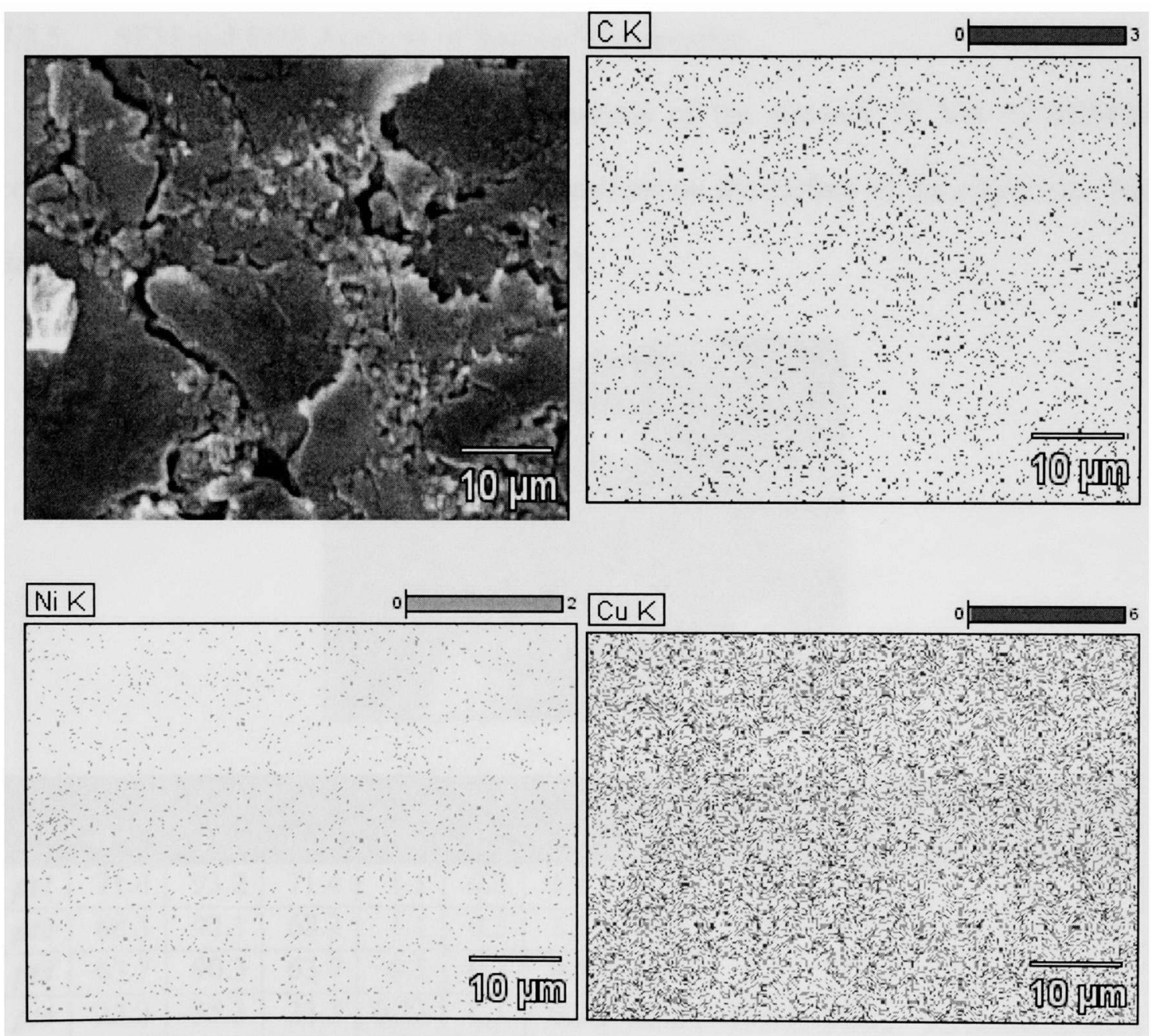




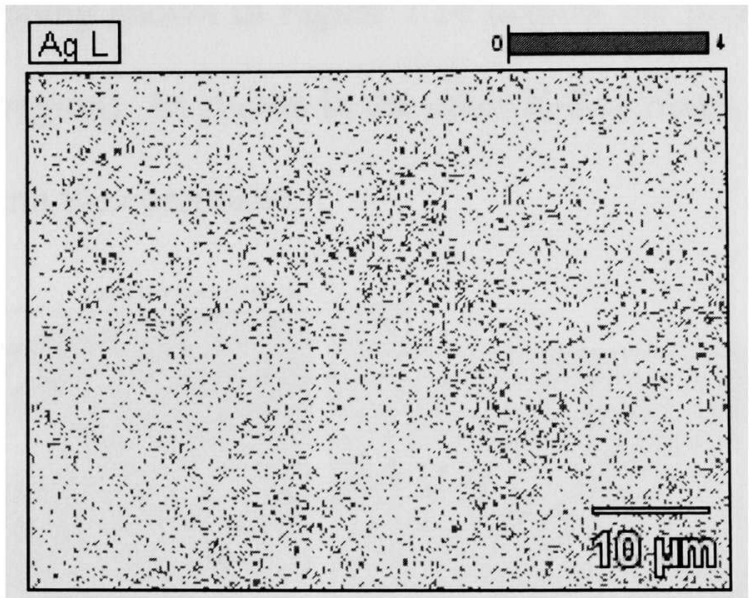

Figure 7.11: X-Ray mapping of un-sintered 8 Vol \% MWCNT composite

\subsubsection{SEM and EDS Analysis of Sintered Composites}

Figure 7.13 illustrates the EDS analysis of the sintered 12 Vol \% MWCNT composite that was performed at various locations on the surface of the composite. Note the presence of MWCNT at points 1,2 and 3.

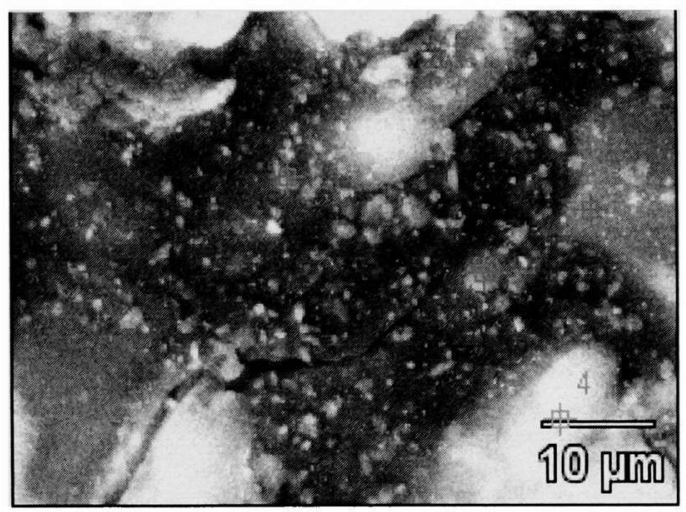

\begin{tabular}{|c|c|c|c|c|c|c|c|c|c|c|c|c|}
\hline & \multicolumn{3}{|c|}{$C-K$} & \multicolumn{3}{|c|}{$N i-K$} & \multicolumn{3}{|c|}{$C u-K$} & \multicolumn{3}{|c|}{$A g-L$} \\
\hline & $W \%$ & A\% & C\% & $W \%$ & A\% & $\mathrm{C} \%$ & $W \%$ & $A \%$ & $C \%$ & $W \%$ & $A \%$ & C\% \\
\hline$p t 1$ & 71.4 & 93.2 & 71.4 & 1.1 & 0.3 & 1.1 & 24.8 & 6.1 & 24.8 & 2.7 & 0.4 & 2.7 \\
\hline$p t 2$ & 68.3 & 92.1 & 68.3 & 1.1 & 0.3 & 1.1 & 26.5 & 6.8 & 26 & 4.0 & 0.6 & 4.0 \\
\hline$p t 3$ & 63.7 & 90.3 & 63.7 & 0.5 & 0.1 & 0.5 & 35.4 & 9.5 & 35.4 & 0.5 & 0.1 & 0.5 \\
\hline$p t 4$ & 23.3 & 55.3 & 23.3 & .23 & 0.1 & 0.3 & 68.2 & 30.6 & 68.2 & 0.6 & 0.2 & 0.6 \\
\hline
\end{tabular}

Fig 7.13: $\quad$ EDS analysis of sintered 16 Vol \% MWCNT composite 
The X-ray mapping shown in Figure 7.14 depicts the presence of carbon (black region), copper (grey region). As shown in the other X-Ray maps, nickel and silver were evenly distributed within the composite.
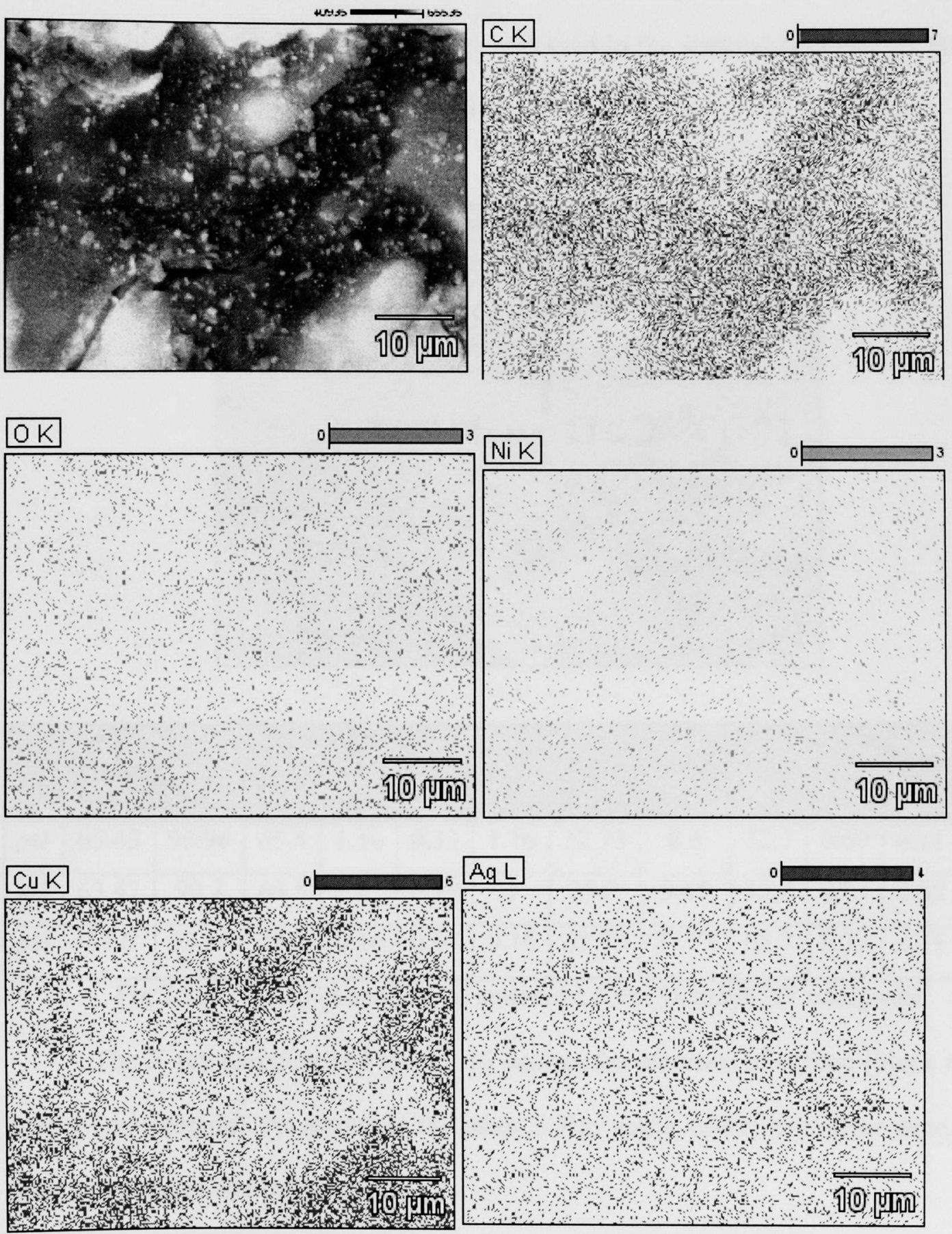

Figure 7.14: X-Ray mapping of sintered 16 Vol\% MWCNT composite 
Figure 7.15 illustrates the EDS analysis of the sintered 12 Vol \% MWCNT composite which was performed at various locations on the surface of the composite. Note the presence of MWCNTs at points 1 and 2. It should be noted that spherical structures high in carbon and nickel were observed in the sintered composites. This was confirmed EDS analysis shown in Fig 7.15.

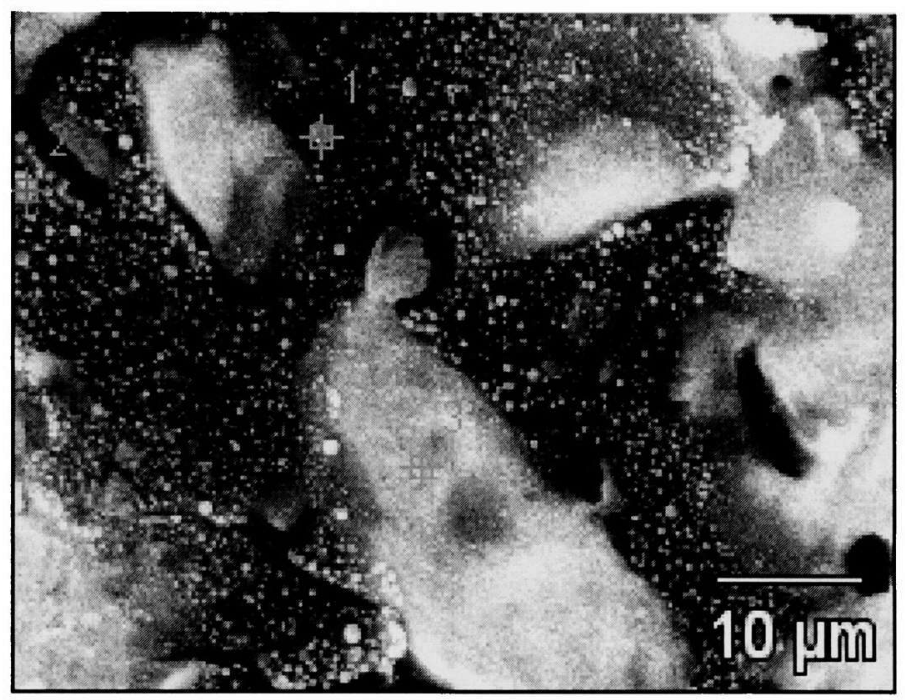

\begin{tabular}{|c|c|c|c|c|c|c|c|c|c|c|c|c|}
\hline & \multicolumn{3}{|c|}{$C-K$} & \multicolumn{3}{|c|}{$N i-K$} & \multicolumn{3}{|c|}{$C u-K$} & \multicolumn{3}{|c|}{$A g-L$} \\
\hline & $W \%$ & $A \%$ & $C \%$ & $W \%$ & $A \%$ & C\% & $W \%$ & $A \%$ & C\% & W\% & $A \%$ & C\% \\
\hline$p t 1$ & 65.43 & 90.96 & 65.4 & 1.16 & 0.33 & 1.16 & 32.73 & 8.6 & 32.7 & 0.69 & 0.11 & 0.69 \\
\hline$p t 2$ & 63.47 & 90.2 & 63.5 & 1 & 0.29 & 1 & 35.2 & 9.45 & 35.2 & 0.33 & 0.05 & 0.33 \\
\hline$p t 3$ & 28.6 & 63.94 & 28.6 & 0.16 & 0.07 & 0.16 & 65.73 & 27.77 & 65.7 & 0.72 & 0.18 & 0.72 \\
\hline
\end{tabular}

Fig 7.15: $\quad$ EDS analysis of sintered $12 \mathrm{Vol} \% \mathrm{MWCNT}$ composite

The X-ray mapping in Figure 7.16 depicts the presence of carbon (black region), copper (grey region). As shown in the other X-Ray maps, nickel and silver were evenly distributed within the composite. 

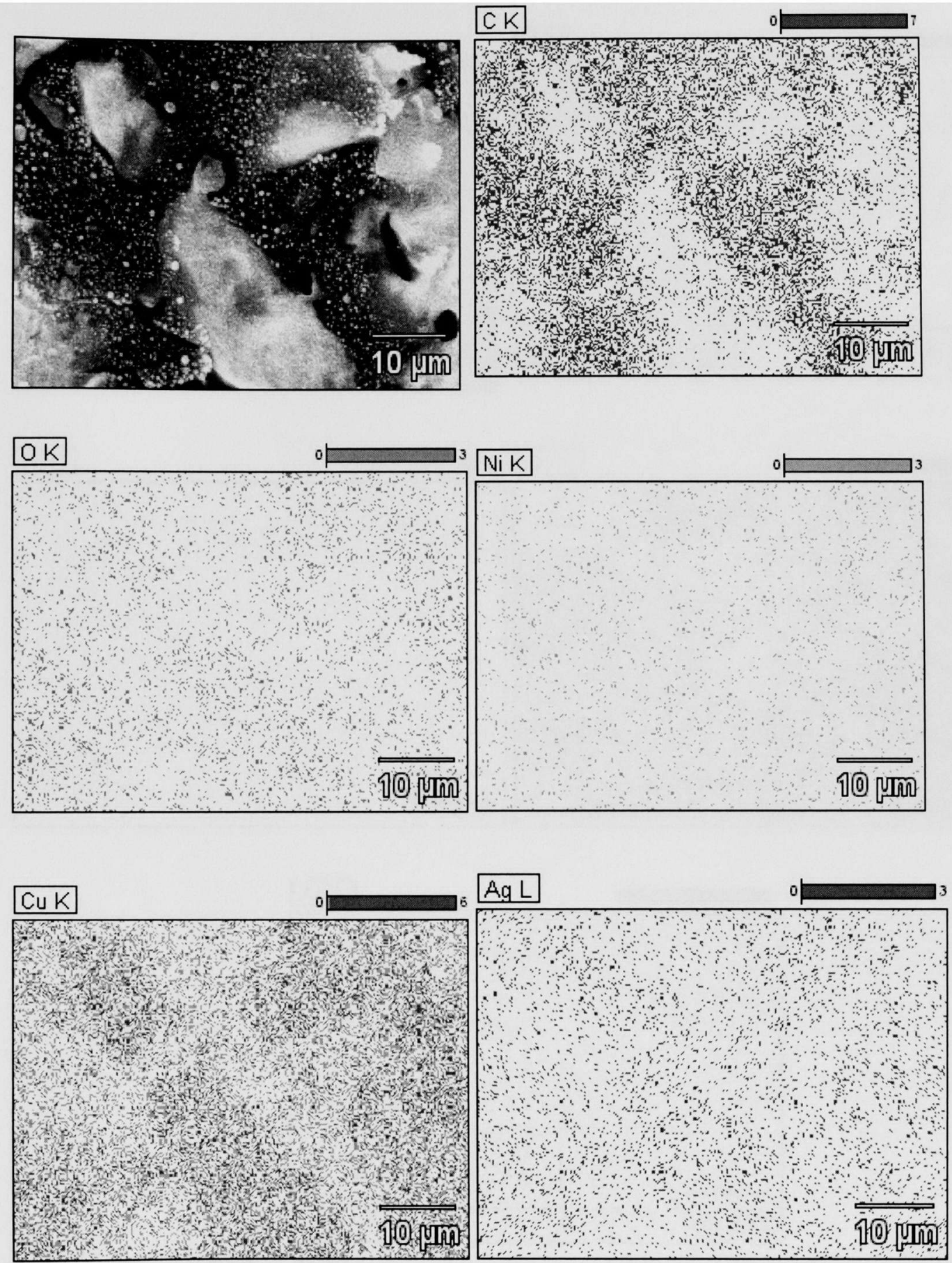

Figure 7.16: X-Ray mapping of sintered $12 \mathrm{Vol} \%$ MWCNT composite

Similarly the X-ray mapping in Figure 7.16 depicts the presence of carbon (black region), copper (grey region). As shown in the other X-Ray maps, nickel and silver were evenly distributed within the composite. 

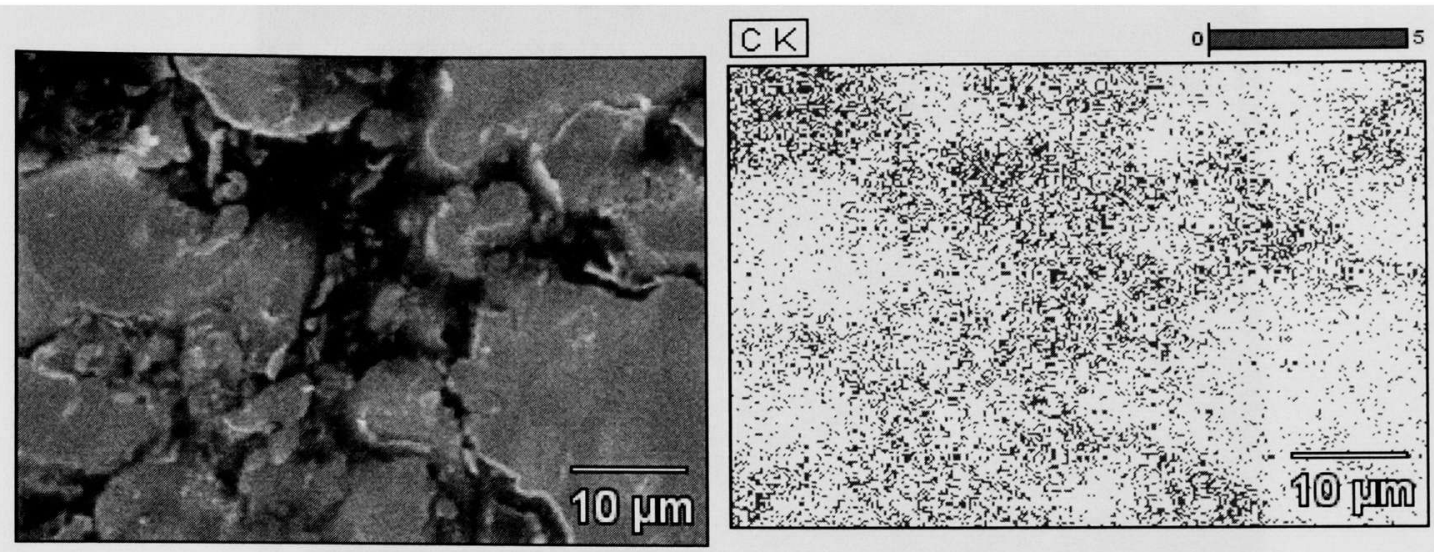

\section{NiK}
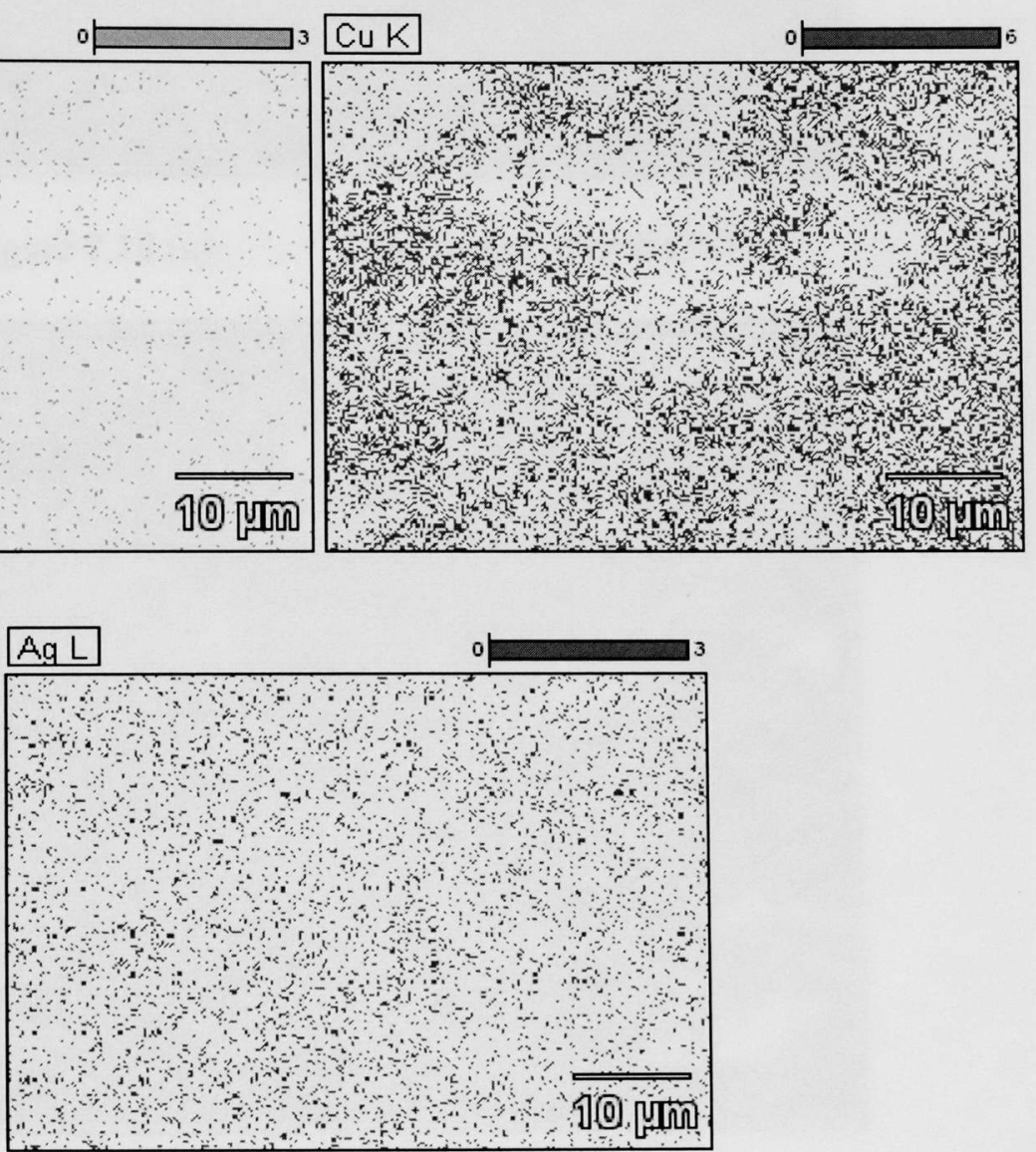

Figure 7.17: X-Ray mapping of sintered 8 Vol\% MWCNT composite

\subsubsection{SEM Images of pure MWCNT}

The SEM photomicrographs of pure MWCNTs are shown in Figures 7.18 (a) and

(b). The measured diameters of the MWCNTs varyied from 10-20 nm. 


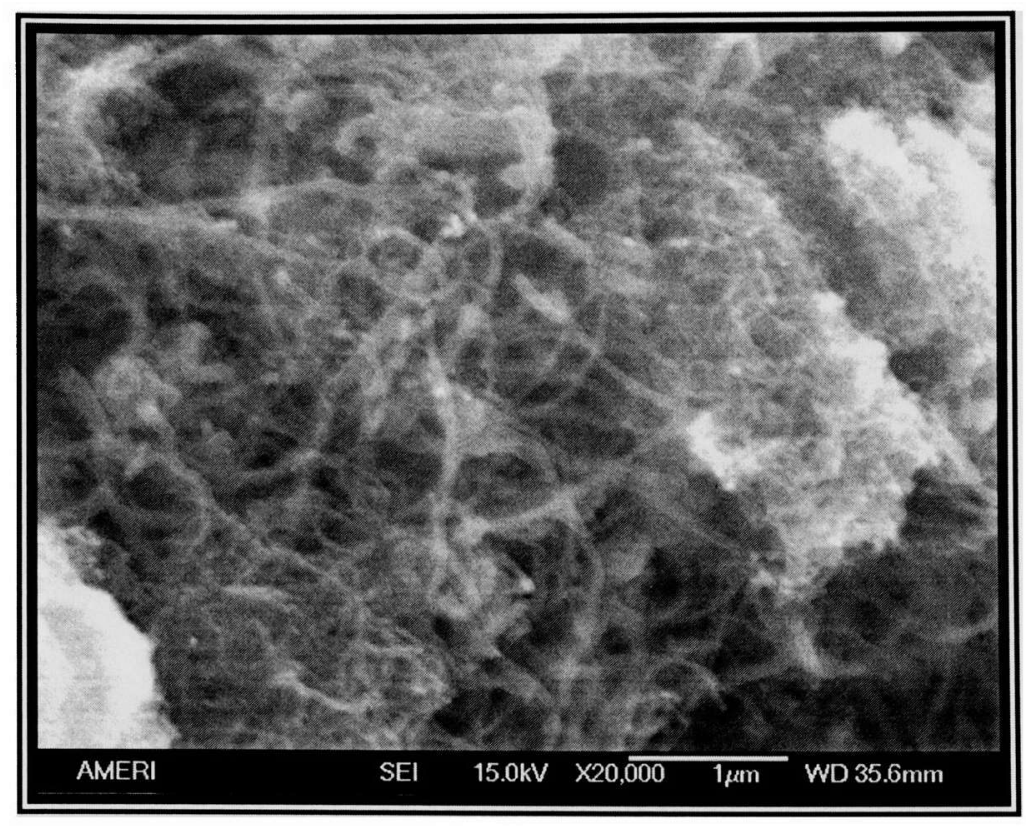

Figure $7.18(a): \quad$ SEM image of MWCNT

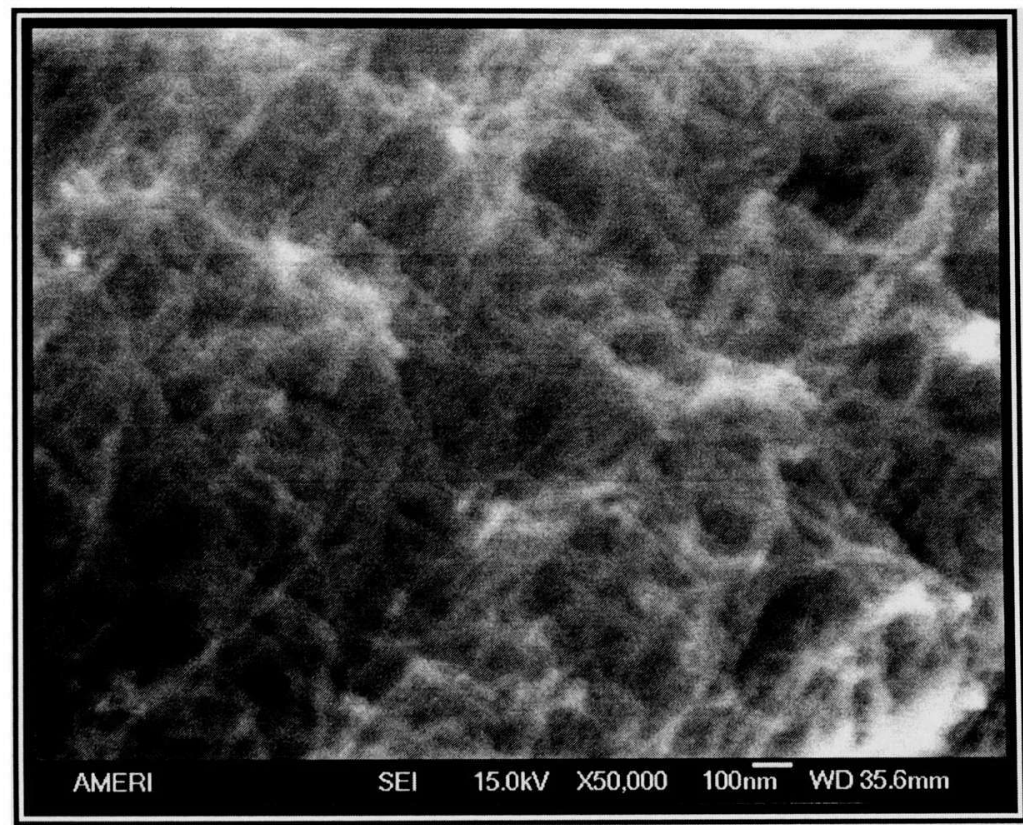

Figure $7.18(\mathrm{~b})$ : $\quad$ SEM image of MWCNT

\subsubsection{SEM Images of Un-sintered Samples}

The Figures 7.19 (a) and (b) showed that MWCNTs act as bridges between the metal matrixes in the composite. It was observed that the diameter of MWCNTs 
increased from $10-20 \mathrm{~nm}$ to $20-25 \mathrm{~nm}$. This was attributed to the formation of a coating of $\mathrm{Cu}, \mathrm{Ag}$ and $\mathrm{Ni}$ on the surface of the MWCNT during ball milling and compaction.

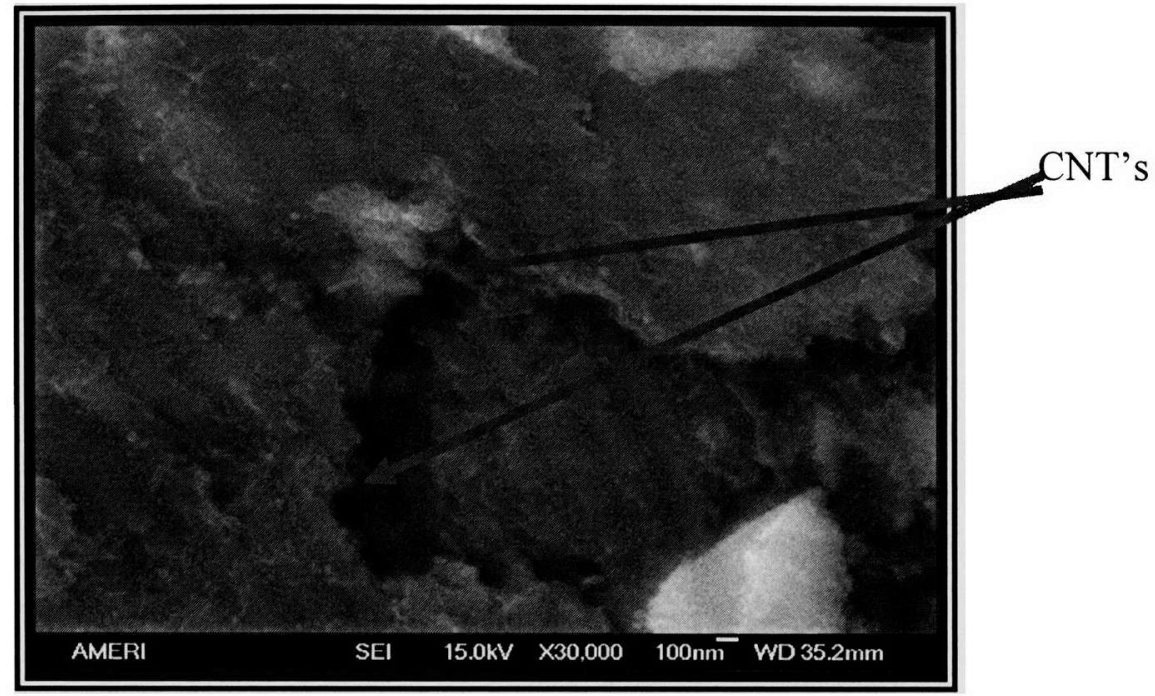

Figure 7.19 (a): $\quad$ SEM image of CuAgNi-MWCNT composite

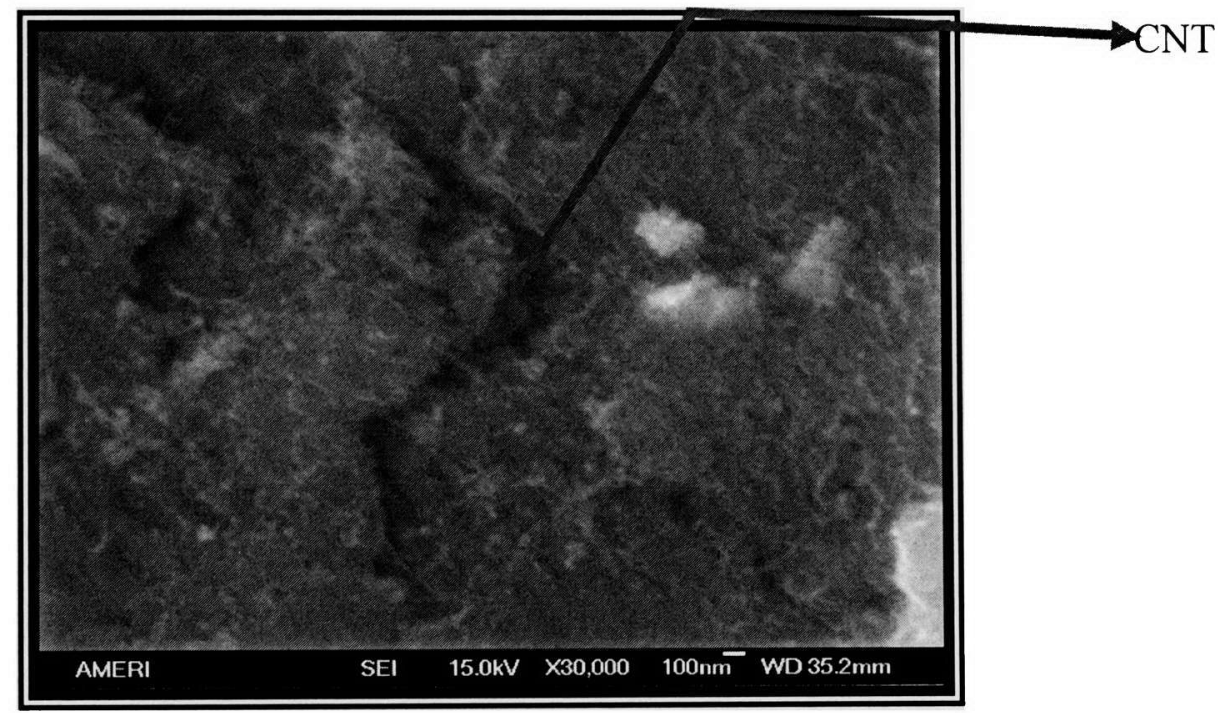

Figure 7.19 (b): $\quad$ SEM image of CuAgNi-MWCNT composite

\subsubsection{SEM Images of Sintered Samples}

The following micrographs (Figures 7.20 (a), (b), (c), (d) and (e)) show with increasing magnification the surface morphology of the sintered composite (note the 
presence of gobules high in nickel and carbon as well as MWCNTs (diameter $33 \mathrm{~nm}$ ) in (d) and (e)). Additionally EDS analysis of the gobules revealed small amounts of Ag and $\mathrm{Cu}$. Moreover, the nickel content in the gobules was higher than that of the matrix. This may be attributed to some carbide formation.

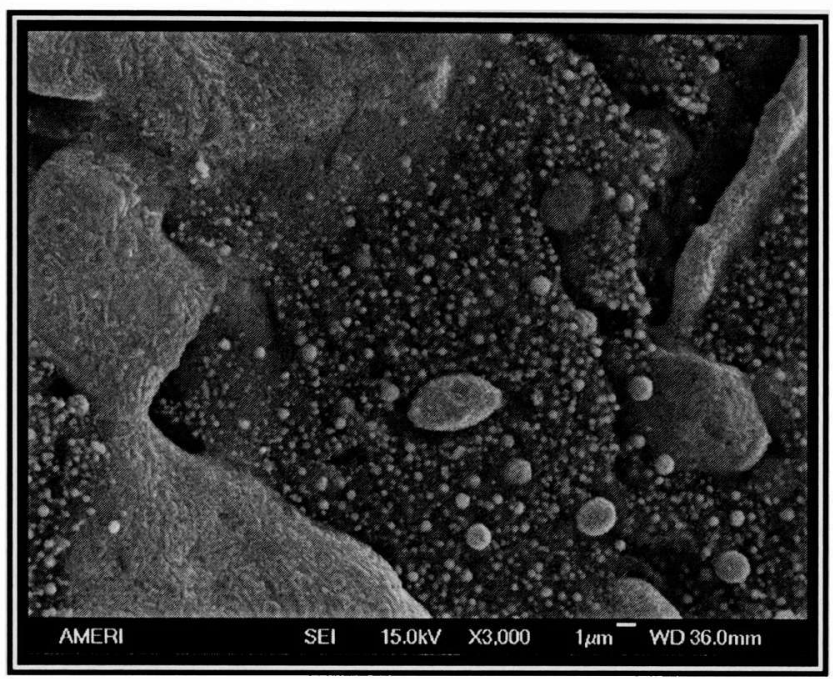

Figure 7.20 (a): $\quad$ SEM image of sintered CuAgNi-MWCNT composite

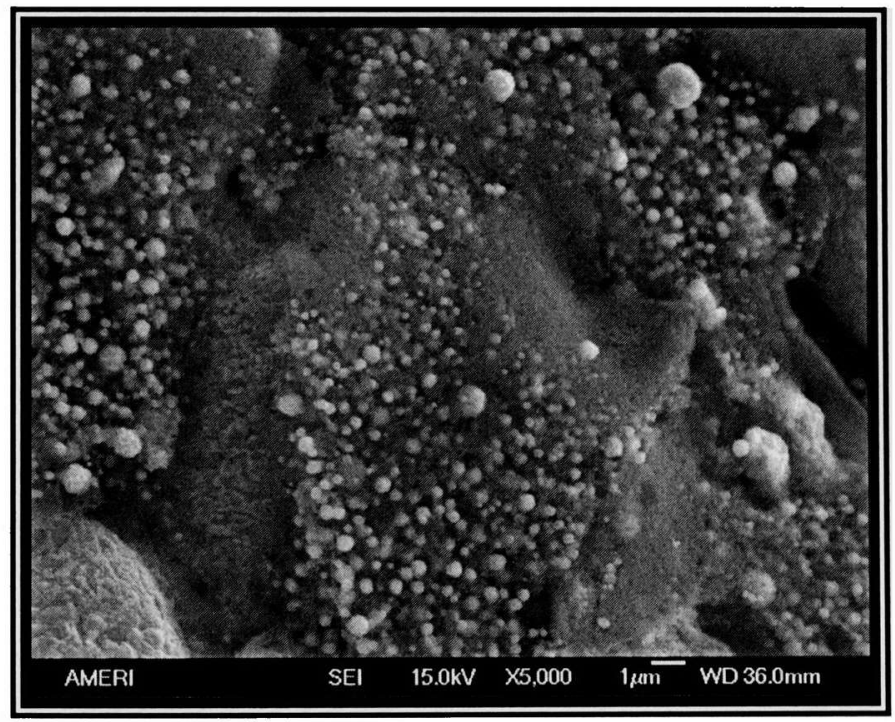

Figure 7.20 (b): $\quad$ SEM image of sintered CuAgNi-MWCNT composite 


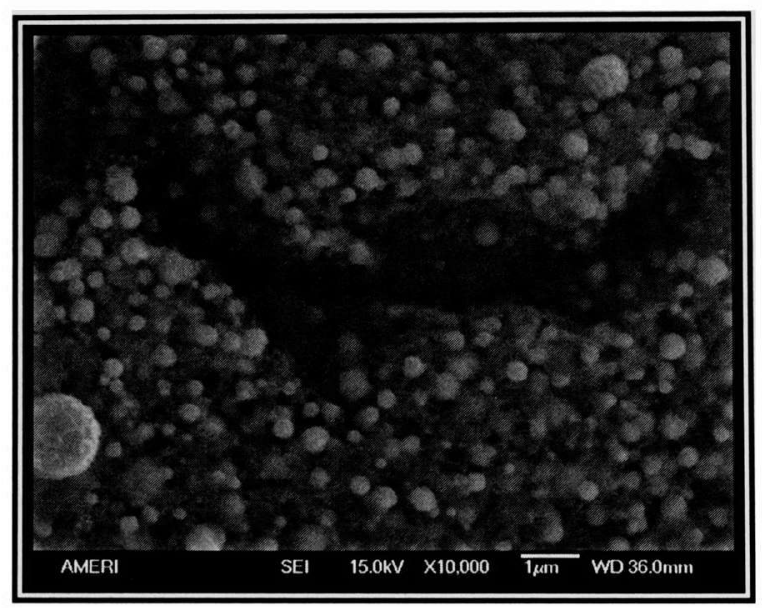

Figure $7.20(\mathrm{c}): \quad$ SEM image of sintered $\mathrm{CuAgNi-MWCNT}$ composite

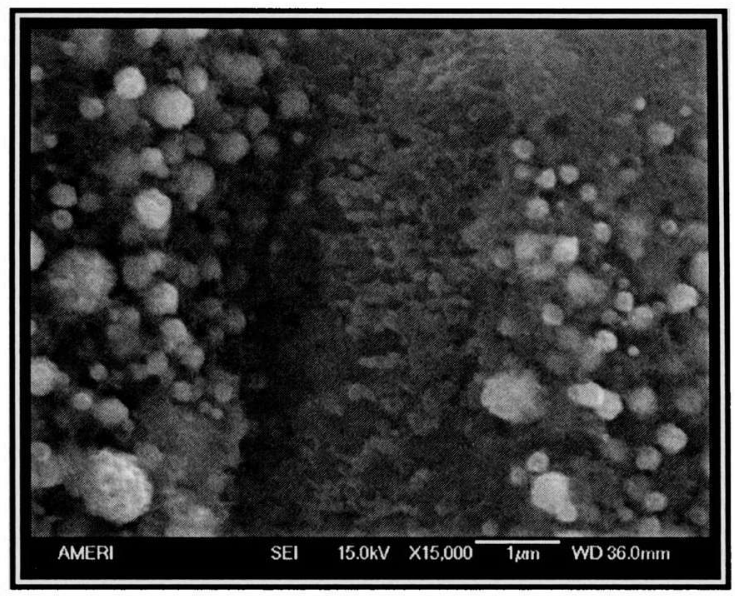

Figure $7.20(\mathrm{~d}): \quad$ SEM image of sintered CuAgNi-MWCNT composite

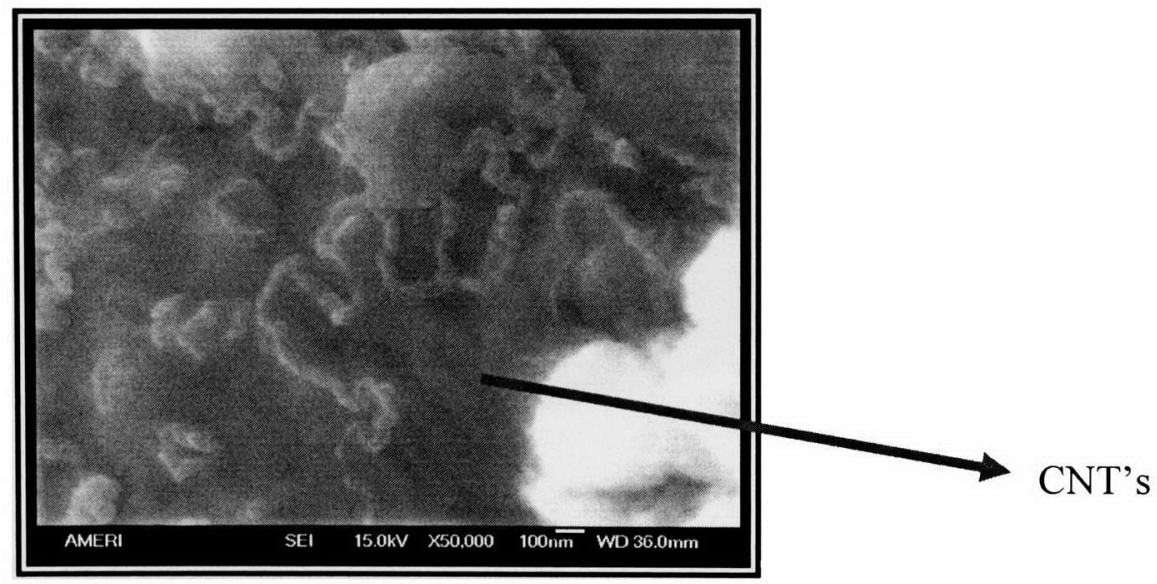

Figure $7.20(\mathrm{e})$ : $\quad$ SEM image of sintered CuAgNi-MWCNT composite 
Figure 7.20 (e) illustrates that some MWCNTs form a chain-like structure with in the composite after sintering.

Based upon the analyses of SEM photomicrographs the composite may be described as having trimodal morphology as depicted in Figure 7.21. The black region was found to be composed of $\mathrm{Cu}, \mathrm{Ag}, \mathrm{Ni}$ and MWCNT. The grey region was composed of predominantly $\mathrm{Cu}$. The white gobules were composed of high amounts of $\mathrm{Ni}$ and $\mathrm{C}$ and small amounts of $\mathrm{Ag}$ and $\mathrm{Cu}$. MWCNT chain-like structures, MWCNT Y-junctions, MWCNT rope anchors and MWCNT bridges were observed within the black region.

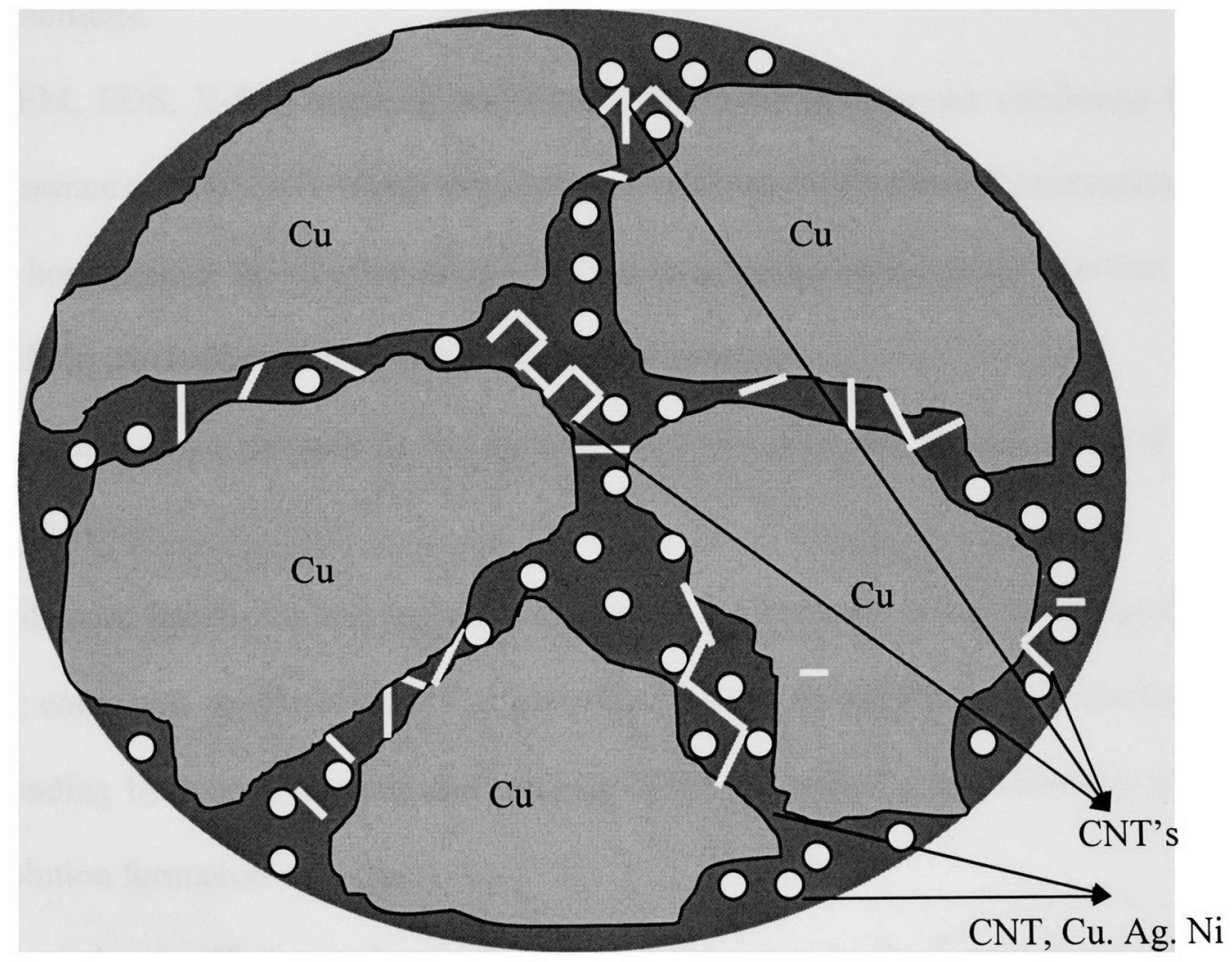

Figure 7.21: Schematic diagram of MWCNT arrangement in the composite 


\section{CONCLUSIONS AND FUTURE WORK}

\subsection{Conclusions}

1. In the current research, $\mathrm{CuAgNi}$ composite reinforced with MWCNT were successfully synthesized by the powder metallurgy route.

2. Compared with Cu-MWCNT composites, CuAgNi-MWCNT composites showed an overall improvement in electrical conductivity with less porosity.

3. An increase in electrical conductivity of $50 \%(8.93 \mathrm{E}+07 \mathrm{~S} / \mathrm{m})$ of that of pure copper was obtained with the composite containing $12 \mathrm{Vol} \%$ MWCNT at STP conditions.

4. SEM, EDS, X-Ray mapping and Raman Spectroscopy analyses confirmed the presence of MWCNTs within the composite with minimal structural destruction.

5. A homogenous distribution of MWCNT was achieved with careful selection of milling parameters with minimal structural destruction.

6. Evidence of an increase in the diameter of MWCNTs was an indication of its wettability.

7. Enhanced interfacial bonding was achieved in the CuAgNi-MWCNT composites as compared to $\mathrm{Cu}-\mathrm{MWCNT}$ composites, where $\mathrm{Ni}$ enhanced the interfacial bonding by rope anchoring and bridging MWCNT with $\mathrm{Cu}$ in addition to solid solution formation with $\mathrm{Cu}$.

8. Any adverse effects on electrical conductivity created by the formation of nickel carbides were compensated by the addition of silver to the Cu-MWCNT composite. 


\subsection{Future Works}

1. Research should be conducted to optimize the amount of Ag and Ni that could be added in order to optimize strength, interfacial bonding and electrical conductivity..

2. The production of higher density composites by HIP could further increase the electrical conductivity of the composite.

3. Mechanical properties such as ductility, hardness and strength of the composites should be determined. 


\section{References:}

[1] O. Hjortstam, P. Isberg, S. Söderholm1, and H. Dai (2004) Can we achieve ultra-low resistivity in carbon nanotube-based metal composites? Applied Physics A: Materials Science \& Processing 78, 1175-1179.

[2] Basic Energy Sciences Workshop and Technical Report on Basic Research Needs for Superconductivity, May 8-10, 2006

[3] A. Modi, N. Koratkar, E. Lass, B. Wei and P. M. Ajayan (2003) Miniaturized gas ionization sensors using carbon nanotubes. Nature 424, 171-174.

[4] E. Meyyappan (2001) Carbon Nanotubes: Science and Application. CRC Press.

[5] S. J. Tans, A. R. M. Verschueren and C. Dekker (1998) Room-temperature transistor based on a single carbon nanotube. Nature, 393, 49-52.

[6] M. M. J. Treacy, T.W. Ebbesen and J.M. Gibson (1996) Exceptionally high Young's modulus observed for individual carbon nanotubes. Nature, 381, 678-680.

[7] Z. L. Wang, R.P. Gao, P. Poncharal, W.A. de Heer, Z.R. Dai and Z.W. Pan (2001) Mechanical and electrostatic properties of carbon nanotubes and nanowires. Mat. Sc. Eng. C, 16, 3-10.

[8] Iijima (1991) Helical microtubules of graphitic carbon. Nature 354, 56-58.

[9] W.A. Heer, A. Chatelain and D.A. Ugarte (1995) Carbon nanotube field-emission electron source, Science 270, 1179-1180.

[10] R.F. Service (1996) Mixing nanotube structures to make a tiny switch, Science 271, $1232-1233$.

[11] J. Jang, J. Bae and S.H. Yoon (2003) A study on the effect of surface treatment of carbon nanotubes for liquid crystalline epoxide-carbon nanotube composite. J. Mater. Chem. 13, 676-681.

[12] J. Liu, M. Shao, X. Chen, W. Yu, X. Liu and Y. Qian (2003) Large-scale synthesis of carbon nanotubes by an ethanol thermal reduction process, J. Am. Chem. Soc. 125 (27), 8088-8089.

[13] P.G. Collins and P. Avouris (2000) Nanotubes for electronics, Sci. Am. 283, 6269.

[14] G.Z. Chen, M.S. Shaffer, D. Coleby, G. Dixon, W. Zhou and D.J. Fray (2000) Carbon nanotube and polypyrrole composites: coating and doping, Adv. Mater. 12, $522-526$. 
[15] H. Zengin, W. Zhou, J. Jin, R. Czerw, D.W. Smith and L. Echegoyen (2002) Carbon nanotube doped polyaniline, Adv. Mater. 14, 1480-1483

[16] A. Peigney, E. Flahaut, C.H. Laurent, F. Chastel and A. Rousset (2002) Aligned carbon nanotubes in ceramic-matrix composites prepared by high-temperature extrusion, Chem. Phys. Lett. 352, 20-25.

[17] L.Q. Jiang and L. Gao (2003) Carbon nanotubes-magnetite nanocomposites from solvothermal processes: formation, characterization, and enhanced electrical properties, Chem. Mater. 15, 2848-2853.

[18] T. Kuzumaki, K. Miyazawa, H. Ichinose, K. Ito (1998) Processing of carbon nanotube reinforced aluminum composite. J. Mater. Res. 13, 2445-9.

[19] C.L. Xu, B.Q.Wei, R.Z. Ma, J. Liang, X.K. Ma, D.H. Wu (1999) Fabrication of aluminium- carbon nanotube composites and their electrical properties. Carbon. 37, $855-8$.

[20] R. George, K.T. Kashyap, R. Rahul, S. Yamdagni (2005) Scripta Mater. 53,1159-63.

[21] F.A. Khalid, O. Beffort, U.E. Klotz, B.A. Keller, P. Gasser, S. Vaucher (2003) Acta. Mater.51, 4575-82.

[22] T. Laha, A. Agarwal, T. McKechnie, S. Seal (2004) Mater. Sci. Eng. A. 381, 24958.

[23] Shobert EI II, editor (1965) Carbon brushes: the physics and chemistry of sliding contacts, New York: Chemical Publishing Co, 5-22.

[24] R.A. Marshall, (1966) Design of brush gear for high current pulses and high rubbing velocities, IEEE Trans Power Apparatus Syst. 11, 1177-88.

[25] J.M. Casstevens, H.G. Rylander and Z. Eliezer (1978) Influence of high velocities on the friction of copper-graphite brushes, Wear. 48, 121-130.

[26] K. T. Lau (2003) Chem. Phys. Lett. 370, 399-405.

[27] A. Peigney (2003) Nat. Mater. 2, 15-16.

[28] E. Flahaut, A. Peigney, Ch. Laurent, Ch. Marliere, F. Chastel and A. Rousset (2000) Acta Mater. 48, 3803-12.

[29] X. Wang, N. P. Padture, H. Tanaka (2004) Nat. Mater. 3, 539-44.

[30] S. J. Sun and M. D. Zhang (1991) J. Mater. Sci. 26, 5762-6. 
[31] D. A. Mortimer and M. Nichlas (1970) J. Mater. Sci. 5, 149-55.

[32] Cha S I, Kim K T, Arshad S N, Mo C B and Hong S H (2005) Adv. Mater. 17, 1377-81.

[33] Zhan G D, Kuntz J D, Wan J and Mukherjee A K (2003) Nat. Mater. 2, 38-42.

[34] Goh C S, Wei J, Lee L C and Gupta M (2006) Nanotechnology 17, 7-12.

[35] Lau K T, Gu C and Hui D (2006) Comp. B 37, 425-36.1

[36] ChenW X, Tu J P, Wang L Y, Gan H Y, Xu Z D and Zhang X B (2003) Carbon 41, 215-22.

[37] Graff R A, Swanson J P, Barone P W, Baik S, Heller D A and Strano M S (2005) Adv. Mater. 17, 980-4.

[38] Bian Z, Pan M X, Zhang Y and Wang W H (2002) Appl. Phys. Lett. 81, 473941.

[39] E. Flahaut (2000) Acta. Mater. 48 (14), 3803.

[40] R.W. Siegel (2001) Scripta. Mater. 44 (9), 2061.

[41] J.-W An and D.-S. Lim (2002) J. Ceram. Processing. Res. 3, 201.

[42] J.-W. An (2003) Wear 255 (1-6), 677.

[43] Cs. Balázsi (2003) Mater. Sci. Eng. C 23 (6-8), 1133.

[44] G.D. Zhan (2003) Nat. Mater 2 (1), 38.

[45] G.-D. Zhan (2003) Appl. Phys. Lett. 83 (6), 1228.

[46] J. Yang and R. Schaller (2004) Mater. Sci. Eng. A 370 (1-2), 512.

[47] X.-T Wang (2004) Nat. Mater 3 (8), 539.

[48] H. Zoz (1995) Attritor technology-latest developments, Mater. Sci. Forum. 179181, 419-424.

[49] C. C Koch (1989) Materials synthesis by mechanical alloying, Annu. Rev. Mater. Sci. 18, 121-143.

[50] D. Basset, P. Matteazzi, and F. Mani (1993) Designing a high energy ball-mill for synthesis nanophase materials in large quantities, Mater. Sci. Eng. A168, 149-152.

[51] J. S. Benjamin (1976) Sci. Am. 234. 40. 
[52] B. S. Murthy, S. Ranganathan (1998) Int. Mater. Rev. 43. 101.

[53] C. Suryanarayana (2001) Progr. Mater. Sci. 46. 1.

[54] J. Ding, T. Tsuzuki and P. G. McCormick (1996) J. Am. Ceram. Soc. 79, 2956.

[55] I. Kerr (1993) Metal Powder Rep., 48, 36-38,.

[56] L. Battezzati, G. Cocco, L. Schiffini and S. Enzo (1988) Materials Science and Engineering, 97, 121-124.

[57] L. Lu and M. O. Lai (1998) Mechanical Alloying. Kluwer Academic Publishers.

[58] Metals Handbook, 10th Ed., v.2, (1990) ASM Handbook Committee, American Society for Metals, USA.

[59] Metals Handbook, 9th Ed., v.2, (1979) ASM Handbook Committee, American Society for Metals, USA.

[60] K. Srikanth, (2004) Study of amorphization in Ni-Ti-Ta system and production of NiTi-Ta alloys through conventional powder metallurgy, M.S. Thesis, Florida Internatonal University, Miami, Florida.

[61] M. John, B. Richard, D. Melissa, (2007) Handbook of sample preparation and handling 10th Ed.

[62] Rex W. Grimshaw (1971) The chemistry and physics of clays and allied ceramic materials $4^{\text {th }} \mathrm{Ed}$.

[63] Lide, David R, CRC Handbook of Chemistry and Physics $75^{\text {th }}$ Ed. Boca Raton, CRC Press, 1994: 12-41

[64] Coppers and Copper alloys for Electrical and Thermal Applications, Copper Development Association. 


\section{APPENDICES}

\section{APPENDIX A}

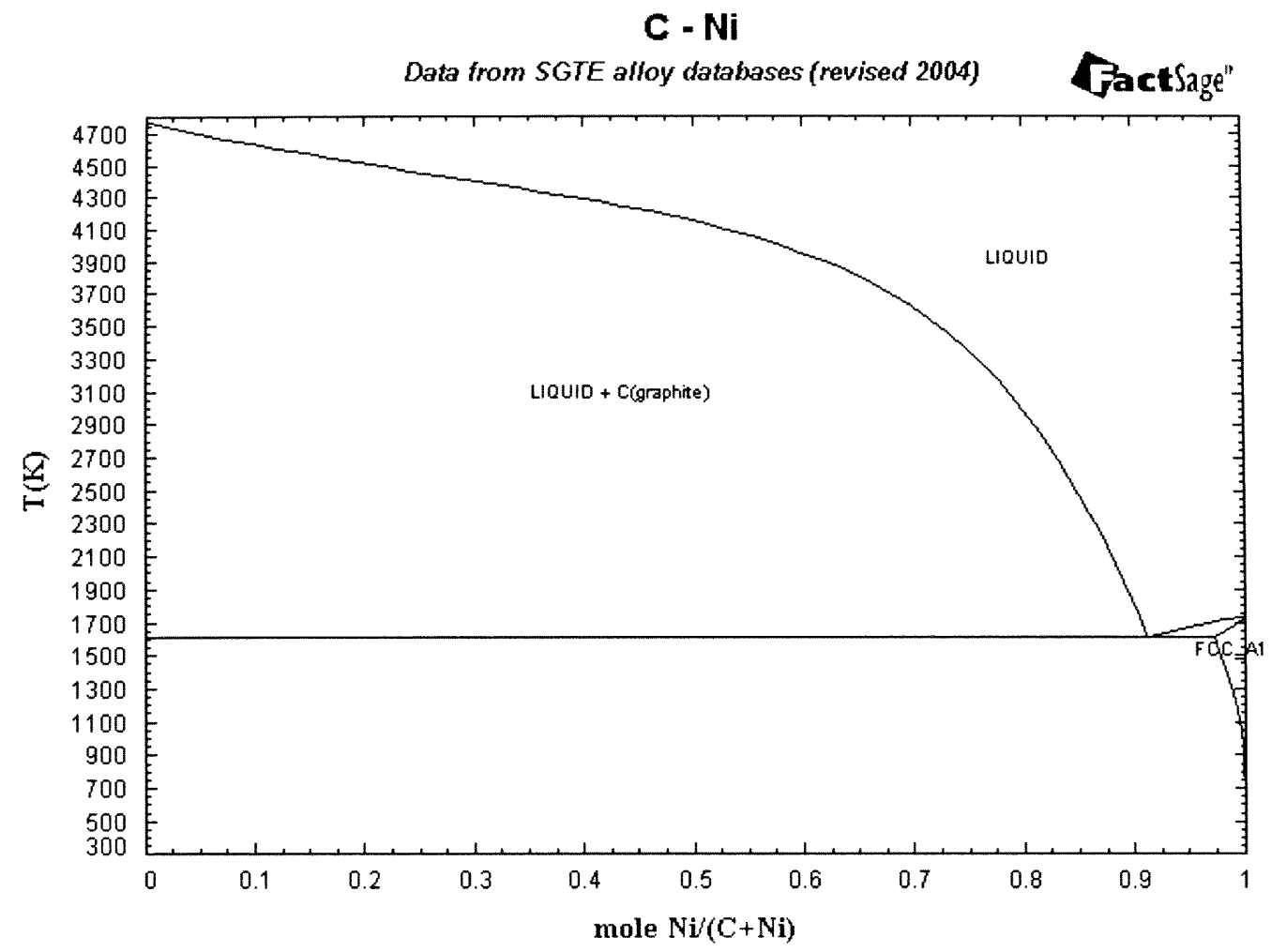




\section{APPENDIX B}

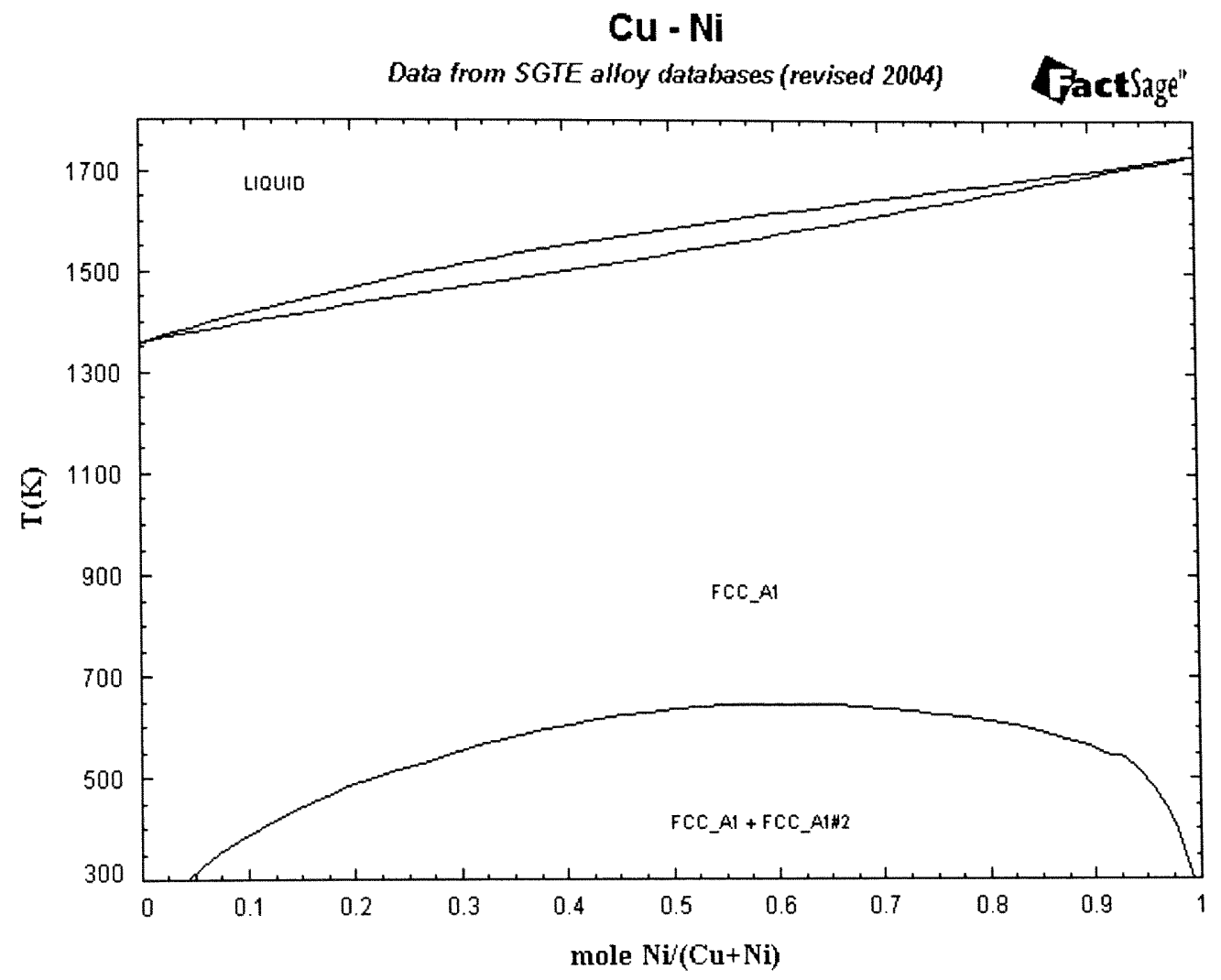


APPENDIX C

Resistivity of Copper

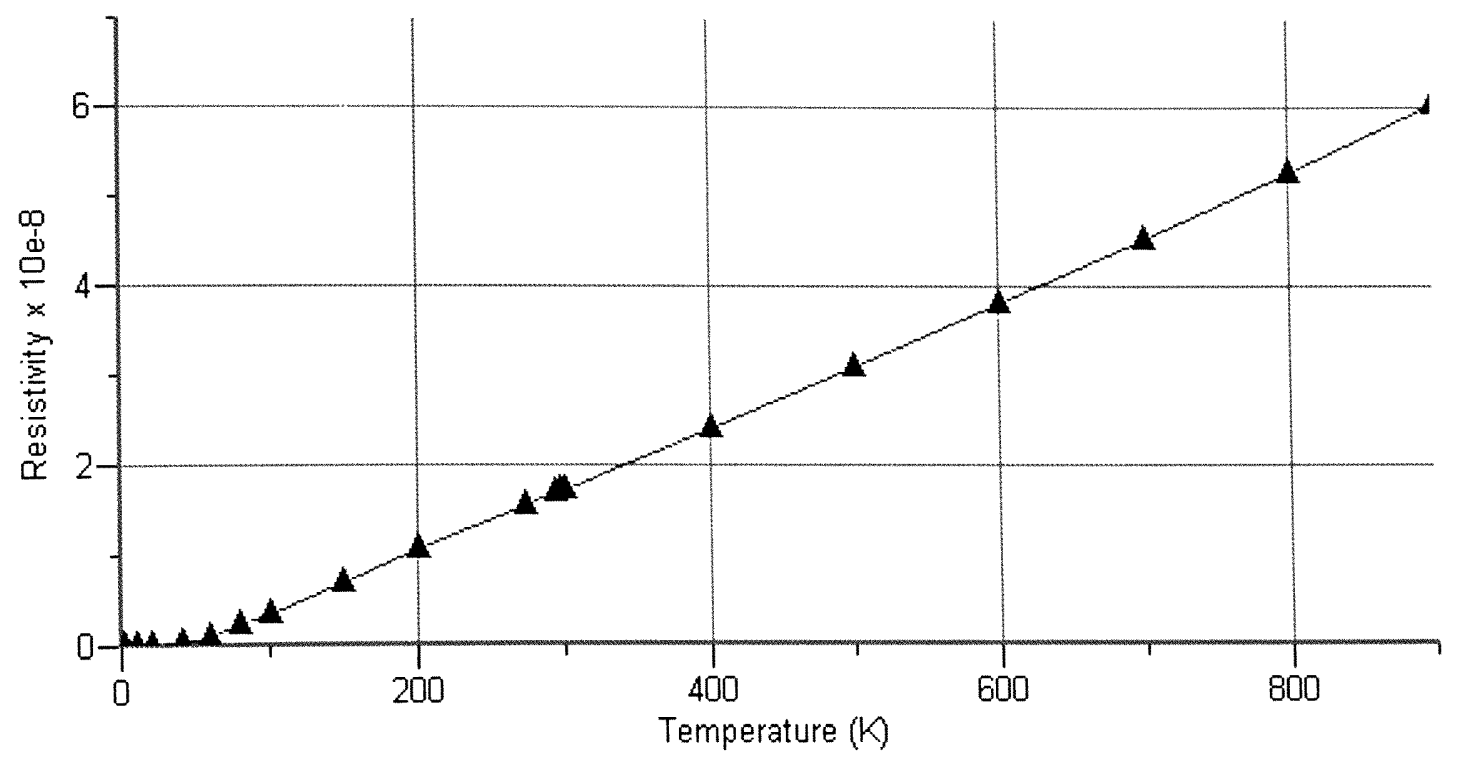

